

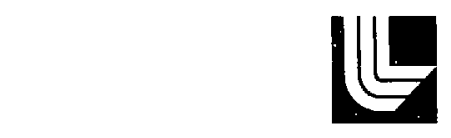

I_AWRENCE I_IVERMORE I_ABORATORY

University of Callornia Livermore, California 94550

UCRL- 52717

\begin{abstract}
MEASUREMENTS, ERROR ANALYSES,
AND CALCULATIONS OF WATER AND STEAM

INDIVIDUAL MASS FLOW RATES,

VELOCITIES, AND RELATED FLOW

PARAMETERS OBTAINED FROM SINGLE-PHASE

AND TWO-PHASE PROTOTYPE TESTS

OF THE PKL INSTRUMENTED SPOOL

PIECES FOR THE U. S. NRC-RSR 3-D PROGRAM
\end{abstract}

\title{
Werner Stein
}

Manuscript date: September 10, 1979

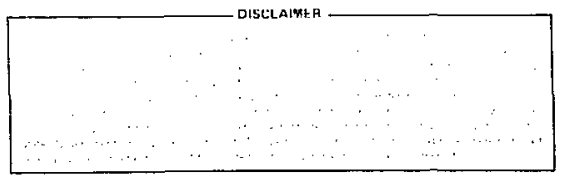


Abstract

Introduction

Spool Piece Measurement Systems and Testing

KWU PKL Test Facility.

PKL Spool Piece Design and Instrumentation.

Spool Piece Physical Description.

Spool Piece Instrumentation

Turbine $\mathrm{Fl}$ owmeter

Drag Screen System

Absolute and Differential Pressure Measurements

Wal1, Fluid, and Șteam Temperature Measurements

Densitometer System .

PKL Spool Piece Data Acquisition System

System Description

Software .

Analytical Relationships.

Engineering Units Conversion.

Flow Parameter Calculations.

Error Analysis

Wyle Laboratories PKL Prototype Spool Test Facility

PKL Prototype Spool Piece Tests

Introduction .

Calibrations

Temperature, Pressure, and Differential Pressure.

Velocity.

Momentum Flux

Densitometer Beam Average Density

Single-Phase Water Tests.

Single-Phase Superheated-Steam Tests

Two-Phase Water and Steam Tests

Overall Spool Head Loss

40-Cycle Limiting Condition Survival Tests 
Summary and Conclusions . . . . . . . . . . . . . 43

References. • . • • • • • • • • • • • • • • 45

Appendix A PKL Spool Piece Drawings (EG\&G Drawing

Nos. 137086, 137087, and 137088) • . . • . • . . 47

Appendix B PKL Spool Piece Test Results Froin Single-Phase Superheated-Steam Tests . . . . . . . . . . 57

Appendix C PKL Spcói Piece Test Results From Two-Phase

Water and Steam Flow Tests . . . . . . . . 69 


\section{LIST OF ILLUSTRATIONS}

1. PKL simulation of a four-loop pressurized water reactor (PWR). . . 4

2. Cold-leg break simulation in the PKL reactor . . . . . . . . 5

3. PKL reflood test facility schematic (cold-leg break) . . . . . 6

4. Flow chart for fluid flow calculations . . . . . . . . 17

5. Flow chart for two-phase parameter calculations . . . . . . 18

6. Two-phase test facility flow diagram . . . . • . . • . 26 
1. Densitometer statistical error analysis

2. Description of numbered boxes in the program flow chart (Fig. 4) and the two-phase parameter flow chart (Fig. 5) . . 19

3. General system characteristics of the Wyle Laboratories Test Facility. • • • • • • • • • • • • • 25

4. Identification of the PKL prototype spool piece tests conducted by Wyle Laboratories . . . . . . . . . . 30

5. PKL prototype spool water-flow test results . . . . . . . 35

6. Overall spool head loss during single-phase water and steam-flow tests • • • • • • • • • • • • • 40 
The operation of the emergency core cooling system and its related steam-binding problems in pressurized water reactors are the subject of a cooperative study by the United States, Germany, and Japan. Lawrence Livermore Laboratory and EG\&G, Inc., San Ramon Operations, are responsible for the design, hardware, and software of the $80.8-\mathrm{mm}$ and $113-\mathrm{mm}$ spool piece measurement systems for the German Primarkreislauf (PKL) Test Facility at Kraftwerk Union in Erlangen, West

Germany. This work was done for the U. S. Nuclear Regulatory Commission, Division of Reactor Safety Research, under its 3-D Technical Support and Instrumentation Program.

Four PKL spool pieces each containing a flow turbine, drag screen, three-beam densitometer, and pressure and temperature probes were constructed and tested to measure single-phase and two-phase steam and water flow parameters. Individual phase velocities, mass flow rates, and densities were calculated from the analytical relationships presented. These calculated and measured parameters were compared to those parameters determined from the test facility instrumentation at Wyie Laboratories. Error analyses were performed, and individual test results were presented for both horizontal and vertical flows. The various flow regimes tested included annular mist, slug, froth, stratified wavy, and homogeneous flow of water or superheated steam. 
The safe operation of nuclear power reactors has been the subject of extensive analyses by scientists and of increasing concern by the general public. The operation of the emergency core cooling system (ECCS) and the related steambinding problems in pressurized water reactors (PWR's) is the subject of a joint cooperative German, Japanese, and United States steam-binding study. The German Primarkreislauf (PKL) Test Facility at Kraftwerk Union (KWU) in Erlangen, West Germany, was constructed to perform loss-of-coolant experiment (LOCE) reflood tests. In order to measure fluid flows during these reflood tests, instrumented pipe spools were developed to measure both single-phase and two-phase water and steam flows in the various coolant loop legs of the PKL reactor. Lawrence Livermore Laboratory and EG\&G, Inc., San Ramon Operations, developed the spool piece measurement system* for the U. S. Nuclear Regulatory Comission, Division of Reactor Safety Research (NRC-RSR), under the 3-D Technical Support and Instrumentation Program.

A total of four spool pieces will be used in the PKL Test Facility. Each spool consists of a flanged pipe that is $830-\mathrm{mm}$ long. Three of the spools have an inside diameter of $80.8 \mathrm{~mm}$, and one spool has an inside diameter of $113 \mathrm{~mm}$. The spools are designed for horizontal installation except for one of the $80.8-\mathrm{mm}$ spools which is designed for vertical installation.

*In this report, the spool piece measurement system is also referred to as a spool piece or a spool. 
Each spool contains the following instruments: '?

- Three-beam densitometer.

- Full-diameter flow turbine.

- Full-diameter drag screen or plate.

- Wall temperature probe.

- Fluid temperature probe.

- Absolute pressure probe.

- Differential pressure probe across the drag screen.

The vertical spool al so contains a superheated-steam probe. Provisions have been made for the installation of a rod endoscope lens system to view flow conditions inside the spools. This system, however, is only for use during prototype testing and is not to be delivered to the PKL Test Facility.

A computer system is supplied with the four spools. Software routines have been devel oped to convert input signals to engineering units and co calculate the following flow parameters:

- Temperature.

- Pressure.

- Vapor and liquid velocities.

- Vapor and liquid densities.

- Void fraction.

- Liquid and vapor mass flow rates.

Prototypes of the spools were tested at a two-phase flow facility constructed at Wyle Laboratories, Norco, California. These tests included the following:

- Single-phase water-flow calibrations.

- Single-phase superheated-steam tests.

- Two-phase flow tests representative of slug, annular mist, froth, and stratified wavy flow conditions.

Testing in both vertical and horizontal flows was accomplished. 
The Wyle Laboratories test facility was equipped with a data acquisition system (DAS) to measure steam and water flow parameters. The wyle DAS also calculated mass and energy balances. A comparison was made of individual phase mass flow rates calculated by the PKL DAS and those determined by the Wyle Laboratories DAS. An error analysis of the data is presented in this report. Data is also presented for overall spool head loss for various tests and spool response to 40 cycles of limiting condition testing.

KWU PKL TEST FACILITY

The German PKL Test Facility at KWU in Erlangen, West Germany was developed to perform LOCE reflood tests. Figure 1 shows the PKL Test Facility modeling of a PWR system. As shown, the PKL reactor is full-scale in the vertical direction and is scaled down on a volume basis by a factor of 134. The reflood tests to be conducted involve coolant loop pipe breaks in the hot legs and also in the cold legs. Figure 2 shows a break in one of the three cool ant loops of the PKL reactor. A schematic of the PKL Test Facility and the relative location of the four spool pieces are shown in Figure 3. 


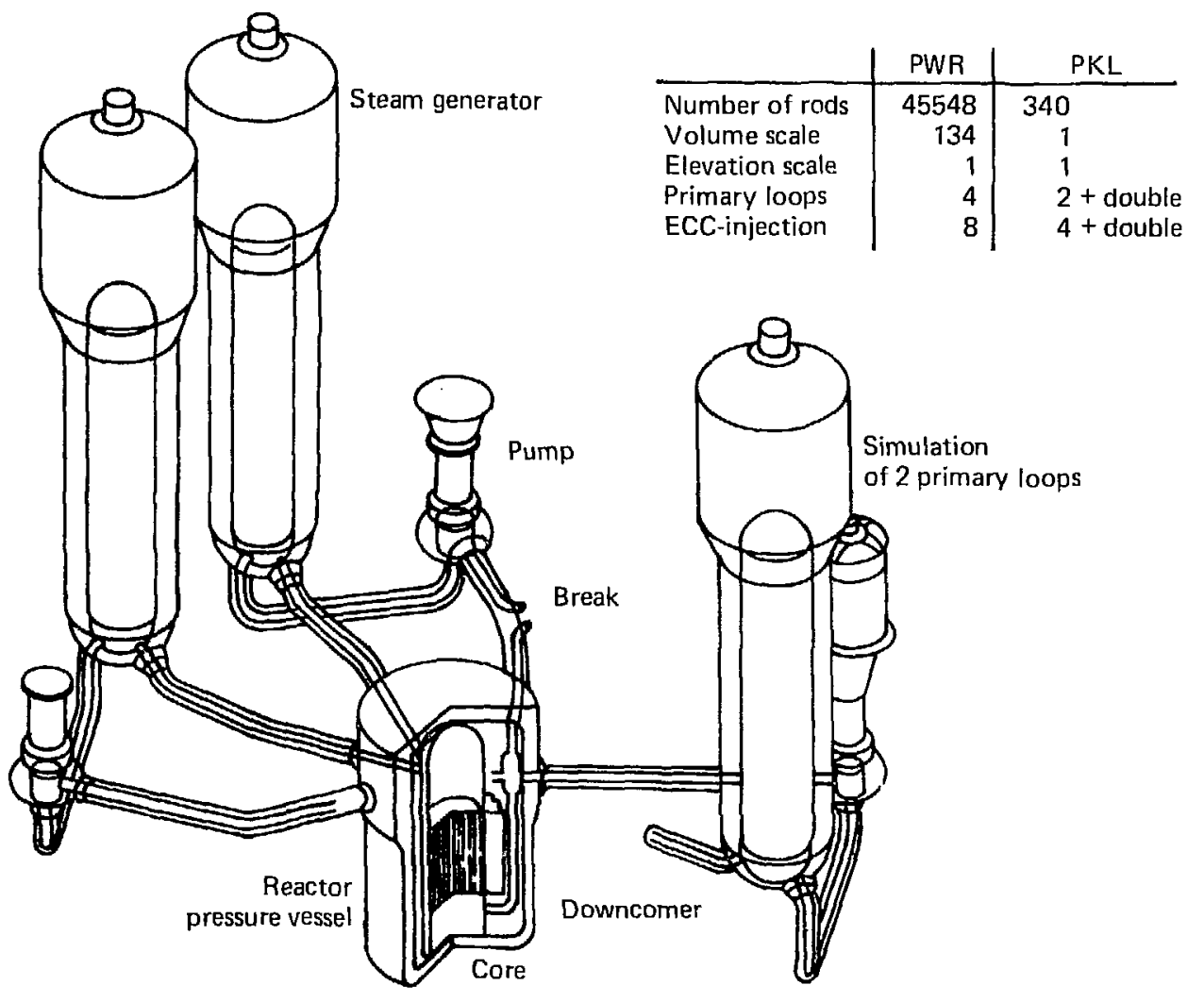

FIG. 1. PKL simulation of a four-loop pressurized water reactor (PWR). 


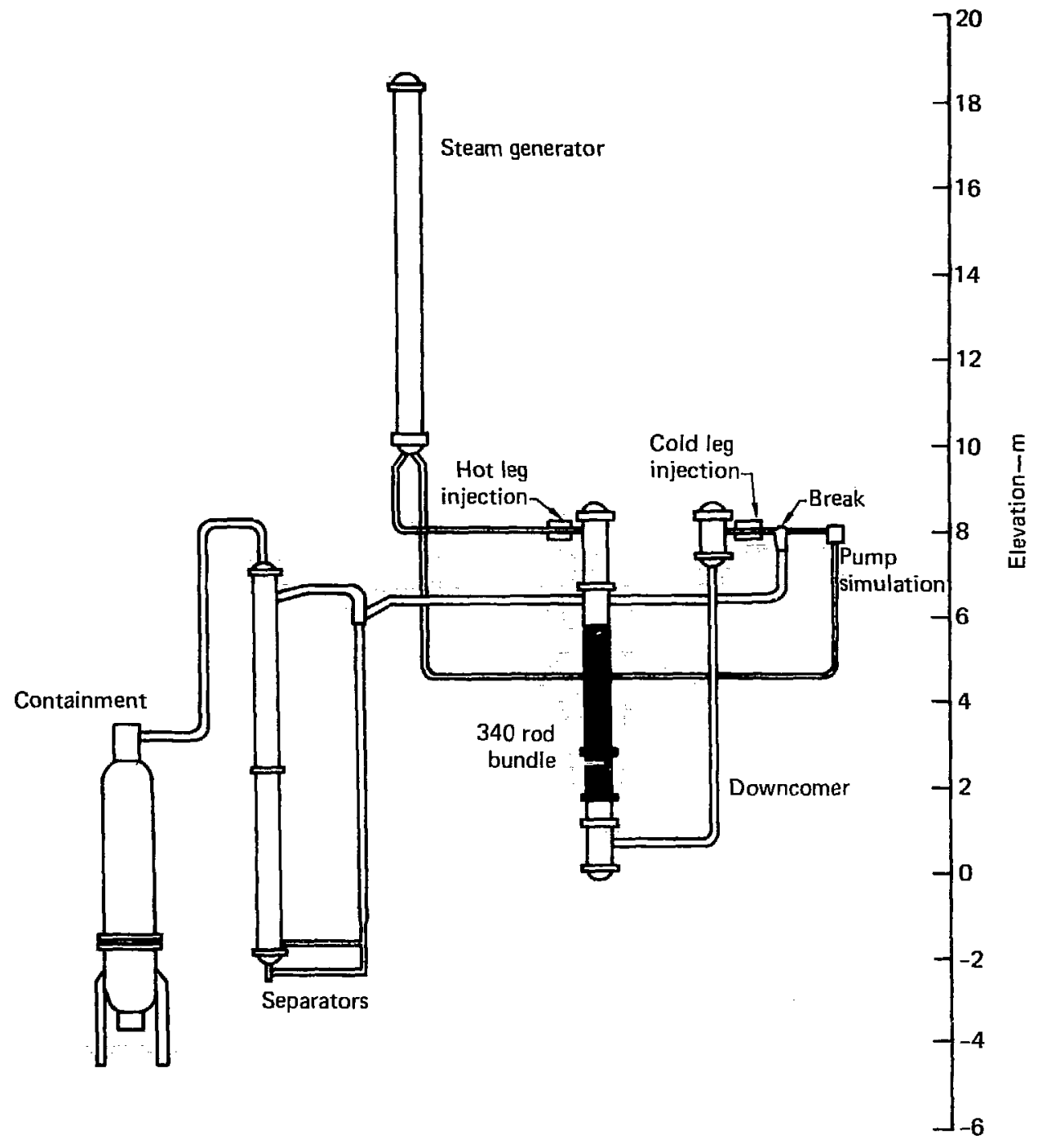

FIG. 2. Cold-leg break simulation in the PKL reactor. 


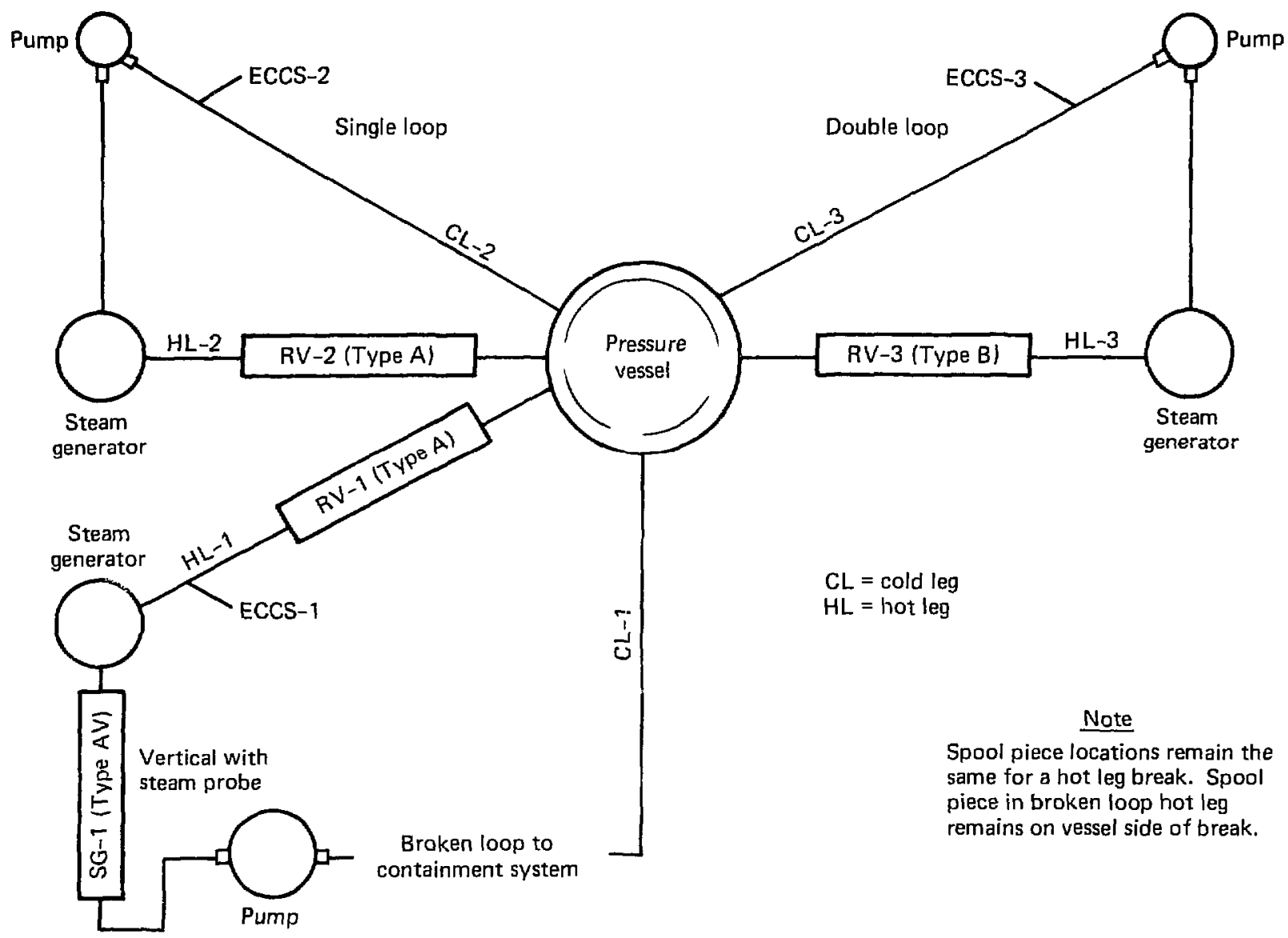

FIG. 3. PKL reflood test facility schematic (cold-leg break). 
The four spools are designed to be mounted to mating flanges in the piping system of the PKL reactor coolant loops.

There are 11 channels of data to be recorded for the three horizontal spools, and there is an additional superheated-steam probe channel to be recorded for the vertical spool.

SPOOL PIECE PHYSICAL DESCRIPTION

The pertinent physical details for each of the spools are given in EG\&G drawing nos. 137086, 137087, and 137088 (refer to Appendix A).

SPOOL PIECE INSTRUMENTATION

A description of the instrumentation used on the spool pieces is given below. For each system, accuracy of $1 \%$ of full scale is required, and a response time of $350 \mathrm{~ms}$ is required for a 10 to $90 \%$ response due to a step excitation.

\section{Turbine Flowmeter}

A whole-duct, bidirectional turbine flowmeter is used to measure volumetric flow in the spool piece. Passage of fluid through the instrument causes the ninebladed rotor to turn at a rate proportional to the flow rate of the fluid. The rotation of the rotor is sensed by two magnetic pickup devices, and the direction of rotation is determined by the phase shift between these two devices. The main components of the flowmeter are a rotor, stainless-steel ball bearings, and flow straighteners attached to both ends of a stationary shaft. The range of operation is from 2 to $60 \mathrm{~m} / \mathrm{s}$. 
A full pipe drag screen or plate is used to measure momentum flux in the spool piece. The drag screen is held in place by a three-pin suspension system. Screen deflections, under flow conditions, are sensed by three variable reluctance transducers. The sum of the three outputs is proportional to the momentum flux. The range of operation is from 70 to $7,200 \mathrm{~kg} / \mathrm{m}-\mathrm{s}^{2}$.

Absolute and Differential Pressure Measurements

The fluid differential pressure is measured across the drag screen, and the absolute pressure is measured upstream of the drag screen. Both measurements are made using water-cooled, water-filled standoff tubes connecting the transducers to the spool piece. The absolute pressure transducer has a range of operation from 0 to $700 \mathrm{kPa}$. The differential pressure transducer has a range of operation from 0 to $7 \mathrm{kPa}$.

Wall, Fluid, and Steam Temperature Measurements

Probes consisting of chromel-alumel thermocouples and an ice-bath reference are used to measure the wall, fluid, and superheated-steam temperatures in the spool piece.

\section{Densitometer System}

The density of single-phase and two-phase flow is measured by a three-beam, low-energy (6-22 keV) x-ray densitometer. The chordal average density is determined by the amount of attenuation of the radiation beam by the fluid in the spool. The densitometer contains a single silicon-lithium-drifted, low-energy photon detector collimated to three sources through the beryllium windows of the spool piece. The radiation sources are Iron-55, Cadmium-109, and Americium-241. The density range of the measurement is from 0.7 ro $1,000 \mathrm{~kg} / \mathrm{m}^{3}$. A densitometer statistical error analysis ${ }^{[1]}$ is given in Table 1 . 
TABLE 1. Densitometer statistical error analysis. [1]

\begin{tabular}{|c|c|c|c|c|c|c|c|c|c|c|c|c|}
\hline \multirow{3}{*}{$\begin{array}{c}\text { Density, } \\
\mathrm{kg} / \mathrm{m}^{3}\end{array}$} & \multirow{3}{*}{\multicolumn{3}{|c|}{$\begin{array}{l}\text { Required } \\
\% \text { error* } \\
\text { of reading }\end{array}$}} & \multicolumn{9}{|c|}{$\%$ error prototype test } \\
\hline & & & & \multicolumn{3}{|c|}{ 80.8-mm horizontal } & \multicolumn{3}{|c|}{ 80.8-mm vertical } & \multicolumn{3}{|c|}{$113-\mathrm{mm}$} \\
\hline & & & & $\mathrm{Fe}^{55}$ & $\mathrm{Cd}^{109}$ & $A m^{24 I}$ & $\mathrm{Fe}^{55}$ & $\mathrm{Cd}^{109}$ & $\mathrm{Am}^{241}$ & $\mathrm{Fe}^{55}$ & $\mathrm{Cd}^{109}$ & $A m 241$ \\
\hline 0.7 & 100.0 & & & 18.9 & 172.0 & 237.0 & 21.0 & 172.0 & 212.0 & 20.1 & 106.0 & 255.0 \\
\hline 1.5 & 46.7 & & & 9.2 & 81.6 & 111.0 & 10.2 & 81.6 & 99.0 & 10.0 & 49.6 & 119.0 \\
\hline 2 & 35.0 & & & 7.1 & 61.3 & 83.3 & 7.8 & 61.3 & 74.5 & 7.8 & 37.3 & 90.0 \\
\hline 3 & 23.3 & & & 5.0 & 41.0 & 55.6 & 5.5 & 41.0 & 49.7 & 5.6 & 24.9 & 60.0 \\
\hline 5 & 14.0 & & & 3.3 & 24.7 & 33.5 & 3.. & 24.7 & 30.0 & 4.9 & 15.1 & 36.2 \\
\hline 10 & 7.0 & & & 2.2 & 12.5 & 17.0 & 2.1 & 12.5 & 15.2 & 3.9 & 7.7 & 18.4 \\
\hline 30 & 2.3 & & & 2.1 & 4.4 & 5.9 & 2.3 & 4.4 & 5.3 & 4.0 & 2.7 & 6.6 \\
\hline 50 & 1.4 & & & 3.7 & 2.8 & 3.7 & 4.1 & 2.8 & 3.3 & 10.3 & 1.8 & 4.2 \\
\hline 70 & 1.0 & 14.3 & (143) & $\star \star *$ & 2.1 & 2.8 & $\star \star$ & 2.1 & 2.5 & $\star \star$ & 1.4 & 3.2 \\
\hline 100 & & 10.0 & $(100)$ & & 1.6 & 2.1 & & 1.6 & 1.9 & & 1.1 & 2.5 \\
\hline 500 & & 2.0 & $(20)$ & & 0.9 & 1.1 & & 0.9 & 1.0 & & 0.9 & 2.0 \\
\hline 1000 & & 1.0 & (10) & & 1.6 & 1.8 & & 1.6 & 1.6 & & 2.6 & 5.7 \\
\hline
\end{tabular}




\section{SYSTEM DESCRIPTION}

The computer system used to monitor and control spool piece testing is an LSI-11 microprocessor system with 32,000 words of memory. The peripherals include the following:

- Line printer.

- CRT terminal (TTY).

- Cartridge tape recorder/reproducer.

- Analog-to-digital converter and multiplexer.

- Three floppy disc drives.

SOFTWARE

Computer programs were developed to allow the acquisition of 50 data channels and to provide the capabilities for:

- Acquiring and storing data on cartridge tape.

- Retrieving data from tape and performing analysis of the data.

- Real-time acquisition and analysis of data.

The analysis of data consists of converting the data signals to engineering units. This is accomplished primarily by a calibration table look-up scheme with straight-1ine interpolation between points. After conversion of the data to engineering units, calculations are made to determine the flow parameters.

\section{ANALYTICAL RELATIONSHIPS}

The major portion of the calculations involves converting the data signals into engineering units, and then using these engineering unit values to detemine the phase velocities, mass flow rate, and void fraction. 
Engineering unit values for the following flow parameters are obtained from cal ibration tables with instrument voltage signals as input:

- Wall temperature $\left({ }^{\circ} \mathrm{C}\right)$.

- Fluid temperature $\left({ }^{\circ} \mathrm{C}\right)$.

- Steam temperature $\left({ }^{0} \mathrm{C}\right)$.

- Fluid absolute pressure (kPa).

- Differential pressure (kPa).

- Flow turbine velocity $(\mathrm{m} / \mathrm{s})$.

- Individual drag screen transducer force (N).

To detemine velocity from the flow turbine, two calibration tables are available. One table is based on a water calibration and the other on an air calibration.

To determine momentum flux from the drag screen, cal ibration tables relating total force to momentum flux are available. The total force is determined by algebraically summing the force values from the three drag transducers. If one of the drag transducers is over-range, an alternate calculation for momentum flux is made. This calculation involves relating differential pressure across the drag screen to the momentum flux.

The density along each of the three beam paths is obtained from the voltage signal. The voltage signal is given by the following relationship: ${ }^{[2]}$

$$
V=V_{\text {off }}+D e^{-C \rho}
$$

where

$$
\begin{aligned}
& v=\text { voltage signal } \\
& v_{\text {off }}=\text { offset vol tage in system }
\end{aligned}
$$


D = calibration constant proportional to the radiation source intensity

C = attenuation cal ibration constant

$\rho \quad=$ average density along beam path.

Engineering unit values for density along each beam are obtained by inverting equation (1) to give:

$$
\rho=\frac{l}{C} \ln \left(\frac{D}{V-V_{\text {off }}}\right) .
$$

\section{Flow Parameter Calculations}

The individual phase parameters calculated include density, velocity, mass flow rate, void fraction, and average pipe cross-sectional density.

Density. The vapor density $\left(\rho_{S}\right)$ and liquid density $\left(\rho_{W}\right)$ are calculated from steam table routines with messured values of fluid temperature and pressure as input. The average cross-sectional density is calculated by fitting a density distribution model to the individual beam density measurements. [3].

Individual Phase Velocities ${ }^{[4]}$ and Void Fraction. The average pipe crosssectional density $\left(\rho_{f}\right)$ of two-phase flow is given by:

$$
\rho_{f}=\alpha \rho_{s}+(1-\alpha) \rho_{W}
$$

where

$$
\begin{aligned}
\alpha & =\text { void fraction } \\
\rho_{S} & =\text { steam density } \\
\rho_{W} & =\text { water density. }
\end{aligned}
$$


The void fraction is given by solving eouation (3) for $\alpha$ :

$$
\alpha=\frac{\rho_{W}-\rho_{f}}{\rho_{W}-\rho_{S}}
$$

The mass flux $\left(G_{f}\right)$ is given by:

$$
G_{f}=\rho_{f} V_{f}=\alpha \rho_{S} V_{S}+(1-\alpha) \rho_{W} V_{W}
$$

where

$$
\begin{aligned}
& V_{f}=\text { average velocity } \\
& V_{S}=\text { vapor velocity } \\
& V_{W}=\text { water velocity. }
\end{aligned}
$$

Solving equation (4) for $V_{f}$ gives:

$$
V_{f}=\frac{G_{f}}{p_{f}}=\frac{\alpha \rho_{S} V_{S}+(1-\alpha) p_{W} V_{W}}{\alpha \rho_{S}+(1-\alpha) p_{W}} .
$$

The momentum flux ( 1 ; is measured from the drag screen and is given by:

$$
I=\alpha \rho_{S} v_{S}^{2}+(1-\alpha)_{\rho_{W}} v_{W}^{2}
$$

The turbine velocity $\left(V_{t}\right)$ is calculated, based on the Rouhani model, [5] as:

$$
V_{t}=\frac{I}{\rho_{f} V_{f}}=\frac{\alpha \rho_{s} V_{S}^{2}+(1-\alpha) \rho_{w} V_{W}^{2}}{\alpha \rho_{S} V_{S}+(1-\alpha) \rho_{w} V_{W}} .
$$

The drag screen and turbine outputs above have been normalized by their cal ibration constants. Another form of this equation shows that the Rouhani model is a flowing quality weighted velocity as follows: 


$$
v_{t}=x v_{S}+(1-x) v_{w}
$$

where

$$
x=\text { flowing quality. }
$$

Solving equations (6) and (7) simultaneously gives the following ind,vidual phase velocities:

$$
\begin{aligned}
& v_{S}=v_{f}+n^{-1 / 2} \Delta V \\
& v_{W}=v_{f}-\eta^{1 / 2} \Delta V
\end{aligned}
$$

where

$$
\begin{aligned}
& \eta=\left(\frac{\alpha}{I-\alpha}\right)\left(\frac{\rho_{S}}{\rho_{W}}\right) \\
& \Delta V=\sqrt{V_{f} V_{t}-V_{f}^{2}} \\
& V_{f}=\frac{I}{\rho_{f} V_{t}} .
\end{aligned}
$$

From an analysis of equations (5) and (7), the turbine velocity $\left(V_{t}\right)$ is equal to or greater than the average fluid velocity $\left(V_{f}\right)$. Therefore, from equation (13), the following is obtained:

$$
\frac{1}{v_{t}^{2}} \leq \rho_{f}
$$

and, in general, the following relationship holds true:

$$
\rho_{S} \leq \frac{I}{v_{t}^{2}} \leq \rho_{f} \leq \rho_{w} .
$$


The relationship given by equation (15) is one of the tests used in the software to determine if the data is satisfactory for proceeding with the calculations. If the test is not found to hold true, then unity slip models are assumed. If the average density $\left(p_{f}\right)$ is found rot to satisfy the test

$$
\rho_{S} \leq \rho_{f} \leq \rho_{W}
$$

then the unity slip velocity (VUS) is assumed to equal the turbine velocity $\left(V_{t}\right)$ and the density $\left(p_{f}\right)$ is set equal to $I / V_{t}^{2}$. If equation (16) is satisfied but the following test,

$$
\rho_{S} \leq \frac{I}{v_{t}^{2}} \leq \rho_{f}
$$

is not satisfied, the VUS is calculated from:

$$
\text { VUS }=\sqrt{\frac{I}{\rho_{\mathrm{f}}}} .
$$

Mass Flow Rate. The calculations for mass flow rates for single-phase and two-phase water and steam flow conditions are presented below.

Single-Phase Water Flow Conditions. The mass flow rate (MW) for single-phase water flow conditions is calculated by the following relationship:

$$
M W=A \times \rho_{W} \times V_{W}
$$

where

$$
\begin{aligned}
& A=\text { pipe cross-sectional area } \\
& V_{W}=\text { water velocity. }
\end{aligned}
$$


$V_{W}$ is calculated from the relationship:

$$
V_{W}=\sqrt{\frac{I}{\rho_{W}}} .
$$

Single-Phase Stean Flow Conditions. For single-phase steam flow conditions, the mass flow rate (MS) is calculated by the following relationship:

$$
M S=A \times \rho_{S} \times V_{S}
$$

where

$$
V_{S}=\text { turbine velocity reading based on an air calibration. }
$$

Two-Phase Flow Conditions. For the case of two-phase flow, the steam mass flcw rates (MS) and the water mass flow rates (MW) are given by:

$$
M S=A \times a \times D_{S} \times V_{S}
$$

and

$$
M W=A \times(1-\alpha) \times \rho_{W} \times V_{W}
$$

where $V_{S}$ and $V_{W}$ are obtained from equations (9) and (10), respectively.

Flow Charts. Flow charts for the calculation steps in the prograin and for the two-phase parameters are shown in Figures 4 and 5 , respectively. A description of each of the numbered boxes shown in Figures 4 and 5 is given in Table 2. The various terms used in these flow charts are explained below:

- $R V=$ spool identification. $R V$ is an input option to allow selection of the spool data to be analyzed. RV $=1$ represents the $80.0-$ inin horizontal spool, RV $=3$ represents the $113-\mathrm{mm}$ diameter spool, and $\mathrm{RV}=4$ represents the vertical $80.8-\mathrm{mm}$ di ameter spool.

- TS = superheated-steam temperature.

- $\mathrm{TF}=$ fluid temperature. 


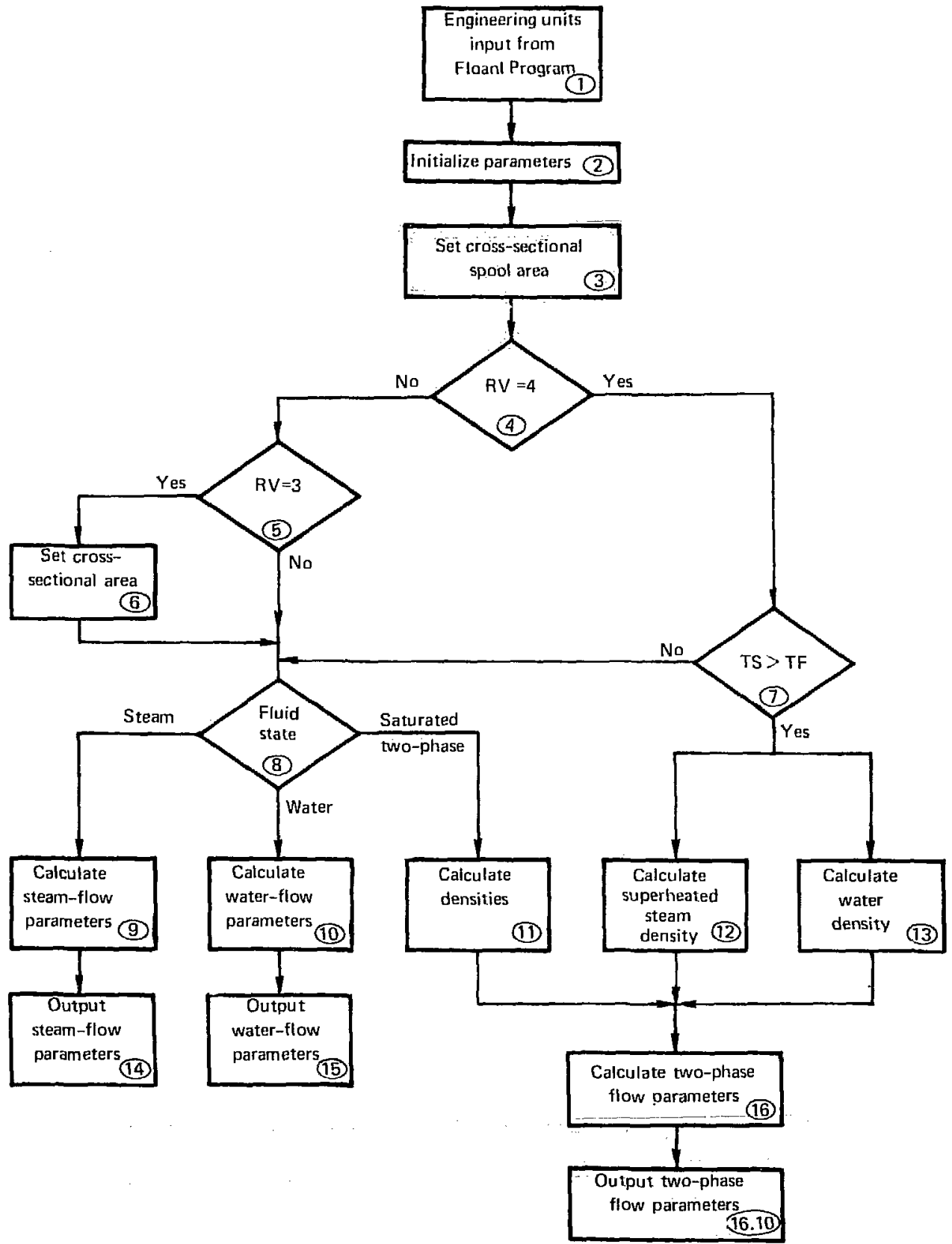

FIG. 4. Flow chart for fluid flow calculations. 


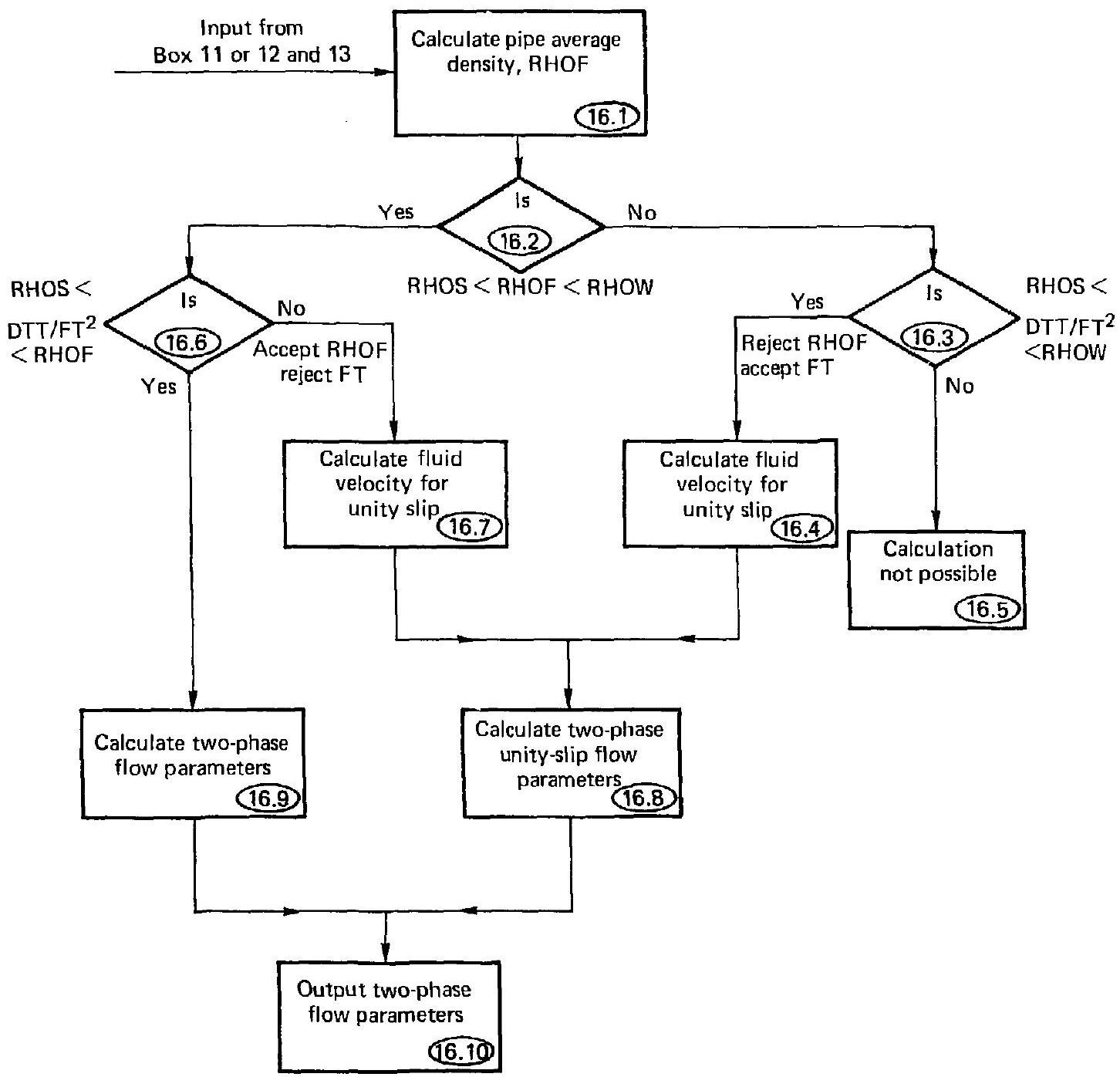

FIG. 5. Flow chart for two-phase parameter calculations. 
- RHOF = average pipe cross-sectional density, $\rho_{f}$.

- RHOS = stearn density, $\rho_{S}$.

- RHOW = water density, $\rho_{W^{*}}$

- $\mathrm{FT}=$ turbine velocity.

- DTr = value of momentum flux (I) as given in equations (6) and (15).

TABLE 2. Description of numbered boxes in the program flow chart (FIG. 4) and the two-phase parameter flow chart (FIG. 5).

BOX

NUMBER DESCRIPTION

1 Program obtains engineering unit values for each of the instrument signals for a designated spool.

2 All flags and flow parameters are zeroed. Pressure and temperature readings are verified to be greater than zero. If either is less than zero, a return without calculation is made to the calling prograin.

3 Spool cross-sectional area is set for the 80.8 -mm diameter spool.

4 A test is made to determine if the spool is no. 4. Spool no. four is spool SC-1 (80.8-m diameter) which is installed in the vertical direction and contains a superheated-steam probe. If this test indicates that the spool is no. 4, then the calculations proceed to Box $\# 7$.

$5 \& 6$ A test is performed to determine if the spool number to be analyzed equals three (113-nm diameter spool). If so, then the cross-sectional area of the spool is modified to reflect the 113-mm diameter spool in Box \#6. 
TABLE 2. Description of numbered boxes in the program flow chart (FIG. 4) and the two-phase parameter flow chart (FIG. 5). (Continued)

BOX

NUMBER

DESCRIPTION

7 A test is made to determine if the superheat-temperature signal (TF) is greater than the fluid temperature by a specified value. This specified value is normally set equal to the sum of the expected full-scale error of the fluid temperature probe and the superheattemperature probe.

8 A calculation is made to determine if the fluid in the spool is subcooled water, superheated steam, or fluid at saturated conditions. This is accomplished by obtaining the maximum and minimum saturation temperatures based on an ab solute pressure reading that has been increased and decreased by the expected error of the pressure probe. These maximum and minimum saturation temperatures are then compared to the measured fluid temperature. If the maximum temperature is greater than the fluid temperature by an amount equal to the expected fullscale error of the fluid temperature probe, then the calculations proceed to Box $\# 9$ and the flow is flagged as "STM", indicating superheated steam. Conversely, if the minimum temperature is less than the fluid temperature minus the expected fluid temperature probe error, then the fluid is deemed subcooled water, flagged "WTR" in the output, and calculations proceed to Box \#10. If the fluid pressure and temperature are close to saturation values, such that the fluid is not deemed superheated steam nor subcooled water, it is assumed to be saturated fluid and calculations proceed to Box \#11.

9 Calculations of single-phase, superheated-steam flow parameters are made. Steam density (RHOS) is calculated. The velocity is set equal to the turbine velocity. The steam mass flow rate (MS) is calculated from equation (21). 
TABLE 2. Description of numbered boxes in the program flow chart (FIG. 4) and the two-phase parameter flow chart (FIG. 5). (Continued)

BOX

NUMBER DESCRIPTION

10 Calculations of single-phase water flow are made. Density (RHOF) is determined. Water velocity (VUS) is calculated by equation (2U). Mass flow is calculated by equation (19).

11 The saturated-steam temperature is determined by the measured pressure. Steam density is determined by the pressure readings and calculated saturation temperature. Water density is calculated from the fluid temperature readings.

$12 \& 13$ The superheated-steam density is calculated from the fluid pressure and superheated steam-probe temperature. Water density is calculated from the fluid temperature readings.

$14 \& 15$ The output of flow parameters is made.

16.1 The average density (RHOF) for two-phase flow is calculated. For the case of saturated water flow, tests are included to recalculate RHOF based on a weighted average of density readings from densitometer beam nos. 1 and 2. This is required because beam no. 3 is not capable of calculating densities above $70 \mathrm{~kg} / \mathrm{m}^{3}$. An option is also provided for the use of beam no. 3 al one to calculate average density (RHOF) when the readings from beam nos. 1 and 2 are below a minimum value.

16.2 A test is made to verify that the average density (RHOF) is between the steam and the water density.

16.3 A range check is made to determine if the turbine and drag screen readings are valid. 
TABLE 2. Description of numbered boxes in the program flow chart (FIG. 4) and the two-phase parameter flow chart (FIG. 5). (Continued)

BOX

NUMBER DESCRIPTION

16.4 The average density (RHOF) value is rejected as being invalid, and density is calculated from the drag screen value of $\rho v^{2}$ divided by the square of the flow turbine velocity. Steam and water velocities are set equal to the velocity detemined from the flow turbine. A flag (USDR) is set to indicate a unity slip condition and that the densitometer readings are rejected.

16.5 No calculations are possible. A fiag (NCAL) is set to reflect this.

16.6 A range check is made to determine if equation (17) is satisfied.

16.7 The velocity readings from the turbine are rejected, but the densitometer readings are accepted. Unity slip steam and water velocities are calculated from equation (18). A flag (USTR) is set in the output to identify the unity slip condition and to indicate that the turbine velocity readings are rejected.

16.8 The void fraction is calculated. Steam, water, and total mass flow rates are calculated from steam table-determined densities, void fraction, cross-sectional spool area, and unity slip flow velocities.

16.9 Calculations are made to deternine individual phase velocities for saturated two-phase steam and water flows from equations (9) and (10).

16.10 The output of the flow parameters is made. 
In order to assess the accuracy of the spool measurements of individual mass flow rates, an error analysis is performed on the data obtained from the prototype single-phase and two-phase flow tests. Data is taken during prototype tests for a period of two minutes at: a rate of five data-channel scans per second. The individual mass flow rates are calculated for each scan, and their standard deviations $(\sigma)$ are calculated from the following equation:

$$
\sigma=\left\{\frac{1}{n-1} \sum_{j=1}^{n}(Y-Y)_{j}^{2}\right\} 1 / 2
$$

where

$$
\begin{aligned}
& P=\text { average of } n \text { values of } Y \\
& Y=\text { calculated mass flow rate. }
\end{aligned}
$$

The average values of individual mass flow rates and their standard deviations are also available from the Wyle Laboratories test facility instrumentation. Using this data, a calculation of the percent difference between the mean results from the wyle Laboratories test facility $\left(\vec{\gamma}_{F a c i l i t y}\right)$ and the PKL spool results $\left(\nabla_{\mathrm{PKL}}\right)$ is made as follows:

$$
\% \text { Difference }=\left(\frac{P_{P K L}-P_{\text {Facility }}}{\bar{Y}_{\text {Facility }}}\right) \times 100
$$

A calculation is also made to determine the deviation $\left(\sigma_{R}\right)$ between the flow rates calculated from the PKL spool piece $\left(Y_{P K L}\right)$ and the mean flow values detemined from the Wyle Laboratories test facility instrumentation. If all of the error is assumed to be random, then this calculation, shown below, would be the same as the standard deviation calculation. It is presented as an aid in analyzing the results and is calculated as shown below: 


$$
\begin{aligned}
& \sigma_{1}=\left\{\frac{1}{n-1} \sum_{j=1}^{n}\left(Y_{\text {PKL }}-P_{\text {Facility }}\right)^{2}\right\}^{1 / 2} \\
& \sigma_{R}=\sqrt{\sigma_{1}^{2}-\sigma_{\text {Facility }}^{2}}
\end{aligned}
$$

where

$$
\begin{aligned}
{ }^{\sigma} \text { Facility }= & \text { standard deviation of of the Wyle Laboratories test facility } \\
& \text { detemined flow rates. }
\end{aligned}
$$


A facility to test the PKL prototype spools was designed and constructed by Wyle Laboratories at their Norco, California, facility. This facility is capable of generating single-phase water flow, superheated-steam flow, and two-phase water and steam flow conditions for testing the horizontal and vertical PKL spools. The pertinent data and a diagram of the test facility are shown in Table 3 and Figure 6 , respectively.

TABLE 3. General system characteristics of the Wyle Laboratories Test Facility.

PARAMETER

Maximum operating pressure

Horizontal distance from mixer to receiver

Cold system activation

Cooldown rate

Mass balance performance

Energy bal ance

Steam venturi metering range

Water turbine metering range

Water venturi metering range

Nominal water circulation pump capacity
150 psig

$28 \mathrm{ft}$

$1-2 \mathrm{hr}$

$2-3^{0} \mathrm{~F} / \mathrm{min}$

$2-5 \%$

$<5 \%$

$0.012-6.0 \mathrm{lb} / \mathrm{s}$

2 - 325 gрл

$300-1000 \mathrm{gpm}$

$400 \mathrm{gpm} 170 \mathrm{ft}$ (each)

Operation of the facility for testing involves the single-phase measurement of steam and of water upstream from a mixer. In the mixer, the steam and water flows are mixed and flow downstream to the PKL spool piece. Downstream of the PKL spool piece, the fluid flow is separated into the steam and water phases in the separator. Steam and water flow rates from the separator, as well as accumulations of water in the separator, are measured. Energy and mas: balances are performed on the system using measured steam and water flows into and from the system to verify the performance of the facility for each prototype test. 


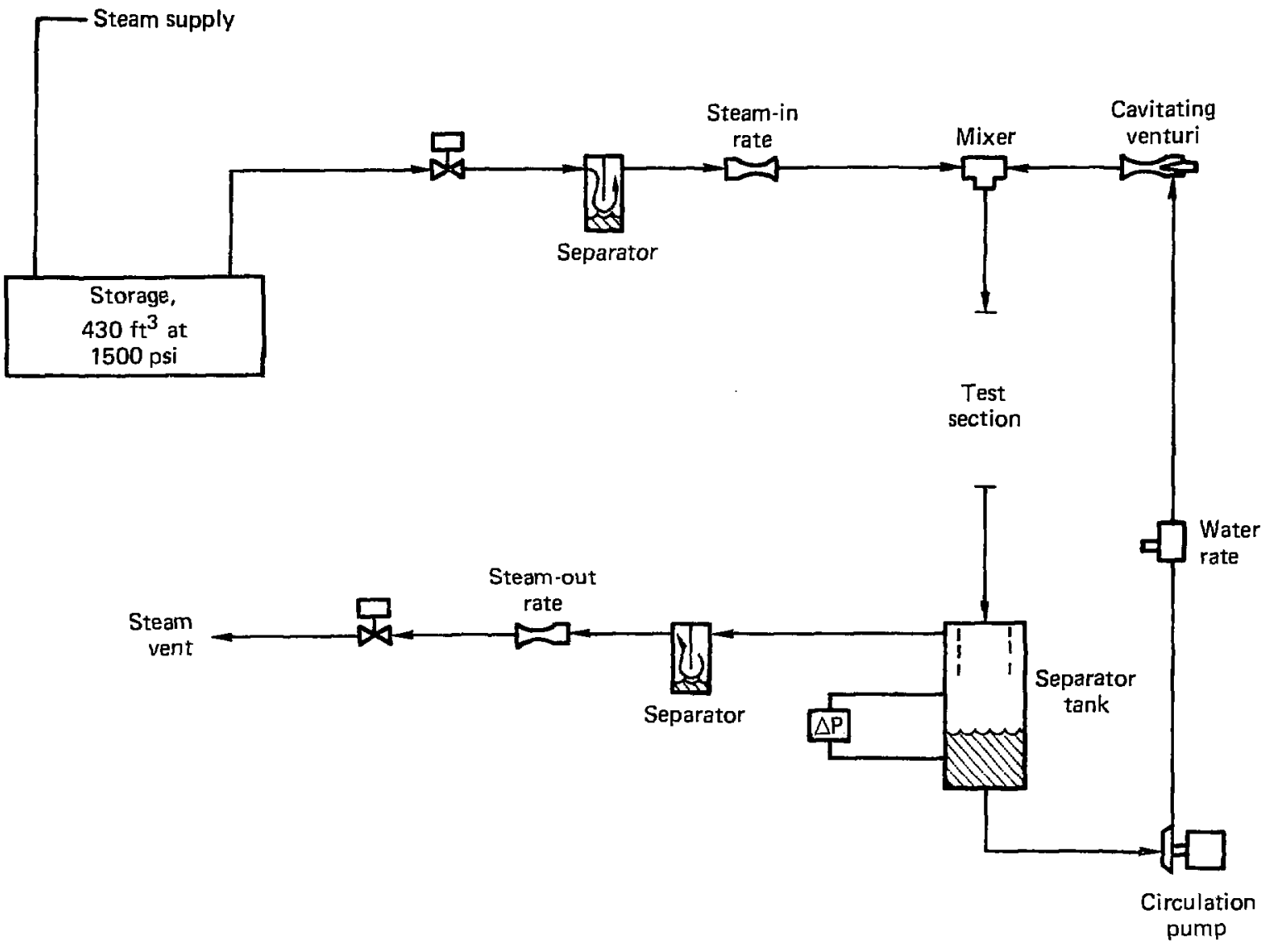

FIG. 6. Two-phase test facility flow diagram. 
A computerized data acquisition system (DAS) performs the calculations. The DAS acquires data over a period of two minutes. It determines the average values of temperature, pressure, and phase mass flow rates. It al so determines the results of mass and energy balance calculations for every ten seconds of the two-minute tests. At the conclusion of the test, a linear regression analysis is performed on the temperature, pressure, and mass flow rate data.

The instrumentation installed in this facility is traceable to standards maintained by the U. S. National Bureau of Standards. 


\section{INTRODUCTION}

The three prototype spools tested at the Wyle Laboratories test facility include the following:

- 80.8-min diameter horizontal spool (designated spool no. 1).

- 113-nm diameter horizontal spool (designated spool no. 3).

- 80.8-mm diameter vertical spool (designated spool no. 4).

The fourth spool (spool no. 2) was not tested since it is essentially identical to spool no. 1 .

Prototype testing involves testing the spools with the flow in the forward direction and al so with the flow in the reverse direction. Changing the flow direction is accomplished by physically turning the spool around in the piping system. Various tests are repeated several times, on different days, and at essentially identical flow conditions to determine repeatability. Spool nos. 1 and 3 are tested under horizontal flow conditions, and spool nc. 4 is tested under vertical flow conditions.

Each of the spools is tested in single-pnase water flow, single-phase superheated steam (approximately 10 to $40^{\circ} \mathrm{F}$ superheat), and two-phase water and steam flows. For two-phase horizontal flow, the flow regines during testing include slug flow, stratified wavy flow, and annular mist flow. For two-phase vertical flow, the flow regimes are slug flow, froth flow, and annular mist flow. The two-phase flow testing is done at saturation conditions and is conducted at three different fluid pressures, i.e., 620, 414, and $207 \mathrm{kPa}$ (90, 60 , and $30 \mathrm{psia}$, respectively). In addition to the above testing, spool nos. 1 and 3 are cycled 40 times between a zero flow condition and a momentum flux condition of $14,400 \mathrm{~kg} / \mathrm{m}-\mathrm{s}^{2}$ to verify their operation without degradation of performance under these conditions. 
In the organization of the test results, the prototype spool tests are each assigned a test number, beginning with no. 1 and ending with no. 302 . Table 4 gives the number of the test, the number of the spool tested, the direction of flow (forward or reverse) during testing, the flow regime during the testing, and any pertinent comments. In the results presented, a positive mass flow rate indicates flow in the forward direction and a negative mass flow rate indicates flow in the reverse direction. Forward flow is defined as flow from the drag screen in the direction of the flow turbine. For the case of vertical flow, forward direction implies downward flow from the drag screen toward the $f 1 \mathrm{~cm}$ turbine. Computer printouts of the test results are given in Appendices $B$ and c.

CALIBRATIONS

Temperature, Pressure, and Differential Pressure

Calibration data relating the instrument electrical signal to engineering unit values of temperature were obtained from vendor data.

Velocity

Calibration data relating the turbine flowneter electrical signal to water velocity were obtained from vendor data. An alternate calibration was obtained from water flow tests perfomed at the wyle Laboratories test facility.

Calibration data relating the instrunent electrical signal to air velocity were obtained from vendor data and were used in determilling turbine velocity in all steam-flow and two-phase flow tests.

\section{Momentum Flux}

Calibration tests of the drag screen system using water were conducted on a ballistic calibrator at Flow Technology, Inc. in Phoenix, Arizona. These calibrations yielded data relating total draz force (the sum of the three drag 
TABLE 4. Identification of the PKL prototype spool piece tests conducted by Wyle Laboratories.

$\begin{array}{lcccc}\text { Test } & \text { Spool } & \text { Flow } & \text { Flow } & \\ \text { no. } & \text { no. } & \text { direction Fluid } & \text { regime } & \text { Comments }\end{array}$

\begin{tabular}{|c|c|c|c|c|c|}
\hline $1-32$ & 1 & Forward & Water & 10 & $1 \emptyset=$ single phase \\
\hline 33 & 1 & Forward & Water & $1 \not$ & Limiting conditions, 40 cycle \\
\hline 36 & 1 & Forward & Wtr \& Stm & Slug & \\
\hline 37 & 1 & Forward & Wtr \& Stm & Ann. & \\
\hline 38 & 1 & Forward & Steam & 10 & \\
\hline 41 & 1 & Forward & Wtr \& Stm & Slug & \\
\hline 42 & 1 & Forward & Wtr \& Stm & Ann. & \\
\hline 43 & 1 & Forward & Wtr \& Stm & Ann. & \\
\hline 44 & 1 & Forward & Wtr \& Stm & Wave & \\
\hline 45 & 1 & Forward & Wtr \& Stm & Ann. & \\
\hline 46 & 1 & Forward & Wtr \& Stm & Ann. & \\
\hline 47 & 1 & Forward & Steam & $1 \emptyset$ & \\
\hline 48 & 1 & Forward & Steam & 10 & \\
\hline 54 & 1 & Forward & Wtr \& Stm & Ann. & \\
\hline 55 & 1 & Forward & Wtr \& Stm & Ann. & \\
\hline 56 & 1 & Forward & Wtr \& Strii & Slug & \\
\hline 57 & 1 & Forward & Wtr \& Stm & Sl ug & \\
\hline 58 & 1 & Forward & Wtr \& Stm & Ann. & \\
\hline 59 & 1 & Forward & Wtr \& Stm & STug & \\
\hline 60 & 1 & Forward & Wtr \& Stm & Wave & \\
\hline 61 & 1 & Forward & Steam & 10 & \\
\hline 62 & 1 & Forward & Steam & 170 & \\
\hline 63 & 1 & Forward & Steam & $1 \emptyset$ & \\
\hline 64 & 1 & Forward & Steam & 10 & \\
\hline 69 & 1 & Reverse & Wtr \& Stm & Ann. & \\
\hline 70 & 1 & Reverse & Wtr \& Stm & Ann. & \\
\hline 71 & 1 & Reverse & Stearn & $1 \emptyset$ & \\
\hline 72 & 1 & Reverse & Steam & $1 \emptyset$ & \\
\hline 73 & 1 & Reverse & Stea!n & $1 \emptyset$ & \\
\hline
\end{tabular}


TABLE 4. Identification of the PKL prototype spool piece tests conducted by Wyle Laboratories. (Continued)

\begin{tabular}{|c|c|c|c|c|c|}
\hline $\begin{array}{l}\text { Test } \\
\text { no. }\end{array}$ & $\begin{array}{c}\text { Spool } \\
\text { no. }\end{array}$ & $\begin{array}{c}\text { Flow } \\
\text { direction }\end{array}$ & Fluid & $\begin{array}{l}\text { Flow } \\
\text { regine }\end{array}$ & Comments \\
\hline 74 & 1 & Reverse & Wtr \& Stin & Wave & \\
\hline 75 & 1 & Reverse & Wtr \& Stin & Slug & \\
\hline 78 & 1 & Reverse & Wtr \& Stm & Wave & \\
\hline 79 & 1 & Reverse & Wtr \& Stm & Slug & \\
\hline 80 & 1 & Reverse & Wtr \& Stm & Ann. & \\
\hline 81 & 1 & Reverse & Wtr \& Stm & Ann. & \\
\hline 82 & 1 & Reverse & Wtr \& Stm & Ann. & \\
\hline 83 & 1 & Reverse & Wtr \& Stm & Ann. & \\
\hline 84 & 1 & Reverse & Wtr \& Stm & Slug & \\
\hline 85 & 1 & Reverse & Wtr \& Stm & Wave & \\
\hline 88 & 1 & Reverse & Stearn & 10 & \\
\hline 89 & 1 & Reverse & Steam & $1 \varnothing$ & \\
\hline 90 & 1 & Reverse & Steam & 10 & \\
\hline 91 & 1 & Reverse & Wtr \& Stin & Ann. & \\
\hline 92 & 1 & Reverse & Wtr \& Stm & Ann. & \\
\hline 93 & 1 & Reverse & Wtr \& Stim & Ann. & \\
\hline 94 & 1 & Reverse & Wtr \& Stm & Slug & \\
\hline 97 & 1 & Reverse & Steam & $1 \emptyset$ & \\
\hline 98 & 1 & Reverse & Wtr \& Stm & Ann. & \\
\hline 99 & 1 & Reverse & Wtr \& 5tri & Slug & \\
\hline $103-121$ & 3 & Reverse & Water & $1 \emptyset$ & \\
\hline $124-142$ & 3 & Forward & Water & $1 \not$ & \\
\hline 143 & 3 & Forward & Water & 10 & Limiting conditions, 40 cycle \\
\hline 146 & 3 & Forward & Wtr \& Stm & S1 ug & \\
\hline 147 & 3 & Forward & Wtr \& Stm & Ann. & \\
\hline 148 & 3 & Forward & Steam & 10 & \\
\hline 152 & 3 & Forward & Wtr \& Stin & Ann. & \\
\hline 153 & 3 & Forward & Wtr \& Stm & Ann. & \\
\hline 154 & 3 & Forward & Wtr \& Stm & Ann. & \\
\hline
\end{tabular}


TABLE 4. Identification of the PKL prototype spool piece tests conducted by Wyle Laboratories. (Continued)

\begin{tabular}{lcccc}
\hline $\begin{array}{l}\text { Test } \\
\text { no. }\end{array}$ & $\begin{array}{c}\text { Spool } \\
\text { no. }\end{array}$ & $\begin{array}{c}\text { Flow } \\
\text { direction }\end{array}$ & Fluid & $\begin{array}{c}\text { Flow } \\
\text { regime }\end{array}$ \\
\hline
\end{tabular}

$\begin{array}{lllll}155 & 3 & \text { Forward } & \text { Wtr \& Stm } & \text { Slug } \\ 156 & 3 & \text { Forward } & \text { Wtr \& Stm } & \text { Wave } \\ 157 & 3 & \text { Forward } & \text { Stealn } & 1 \emptyset \\ 158 & 3 & \text { Forward } & \text { Wtr \& Stm } & \text { Ann. } \\ 159 & 3 & \text { Forward } & \text { Wtr \& Stm } & \text { Ann. } \\ 161 & 3 & \text { Forward } & \text { Wtr \& Stm } & \text { Slug } \\ 162 & 3 & \text { Forward } & \text { Wtr \& Stm } & \text { Ann. } \\ 163 & 3 & \text { Forward } & \text { Wtr \& Stm } & \text { Ann. } \\ 164 & 3 & \text { Forward } & \text { Wtr \& Stm } & \text { Slug } \\ 170 & 3 & \text { Forward } & \text { Wtr \& Stm } & \text { Slug } \\ 171 & 3 & \text { Forward } & \text { Wtr \& Stm } & \text { Ann. } \\ 172 & 3 & \text { Forward } & \text { Steatn } & 1 \emptyset \\ 175 & 3 & \text { Reverse } & \text { Wtr \& Stm } & \text { Ann. } \\ 176 & 3 & \text { Reverse } & \text { Wtr \& Stm } & \text { Ann. } \\ 177 & 3 & \text { Reverse } & \text { Wtr \& Stm } & \text { Wave } \\ 178 & 3 & \text { Reverse } & \text { Wtr \& Stm } & \text { SI ug } \\ 179 & 3 & \text { Reverse } & \text { Wtr \& Stm } & \text { Wave } \\ 180 & 3 & \text { Reverse } & \text { Wtr \& Stin } & \text { Ann. } \\ 131 & 3 & \text { Reverse } & \text { Wtr \& St!n } & \text { Ann. } \\ 182 & 3 & \text { Reverse } & \text { Wtr \& StIm } & \text { Slug } \\ 183 & 3 & \text { Reverse } & \text { Steam } & 1 \emptyset \\ 188 & 3 & \text { Reverse } & \text { Wtr \& Stm } & \text { Wave } \\ 189 & 3 & \text { Reverse } & \text { Wtr \& Stm } & \text { Ann. } \\ 190 & 3 & \text { Reverse } & \text { Wtr \& Stm } & \text { Slug } \\ 191 & 3 & \text { Reverse } & \text { Wtr \& Stm } & \text { Ann. } \\ 192 & 3 & \text { Reverse } & \text { Wtr \& Stm } & \text { Slug } \\ 193 & 3 & \text { Reverse } & \text { Wtr \& Stm } & \text { Ann. } \\ 194 & 3 & \text { Reverse } & \text { Steam } & 1 \emptyset \\ 233-244 & 4 & \text { Forward } & \text { Water } & 1 \emptyset\end{array}$


TABLE 4. Identification of the PKL prototype spool piece tests conducted by Wyle Laboratories. (Continued)

$\begin{array}{lcccc}\text { Test Spool flow } & & \text { Flow } \\ \text { no. } & \text { no. direction fluid } & \text { regime comments }\end{array}$

$\begin{array}{lllll}245 & 4 & \text { Forward } & \text { Wtr \& Stm } & \text { Ann. } \\ 246 & 4 & \text { Forward } & \text { Wtr \& Stm } & \text { Ann. } \\ 247 & 4 & \text { Forward } & \text { Wtr \& Still } & \text { Froth } \\ 248 & 4 & \text { Forward } & \text { Wtr \& Stm } & \text { Froth } \\ 249 & 4 & \text { Forward } & \text { Wtr \& Stm } & \text { Ann. } \\ 250 & 4 & \text { Forward } & \text { Wtr \& Stm } & \text { Ann. } \\ 251 & 4 & \text { Forward } & \text { Wtr \& Stm } & \text { Slug } \\ 252 & 4 & \text { Forward } & \text { Steam } & 1 \emptyset \\ 258 & 4 & \text { Forward } & \text { Wtr \& Stm } & \text { Froth } \\ 259 & 4 & \text { Forward } & \text { Wtr \& Stm } & \text { Froth } \\ 260 & 4 & \text { Forward } & \text { Wtr \& Stm } & \text { Slug } \\ 261 & 4 & \text { Forward } & \text { Wtr \& Stm } & \text { Froth } \\ 262 & 4 & \text { Forward } & \text { Wtr \& Stm } & \text { Ann. } \\ 263 & 4 & \text { Forward } & \text { Wtr \& Stm } & \text { Ann. } \\ 264 & 4 & \text { Forward } & \text { Steam } & 1 \emptyset \\ 267-273 & 4 & \text { Reverse } & \text { Water } & 1 \emptyset \\ 282 & 4 & \text { Reverse } & \text { Wtr \& Stm } & \text { Ann. } \\ 283 & 4 & \text { Reverse } & \text { Wtr \& Stm } & \text { Ann. } \\ 284 & 4 & \text { Reverse } & \text { Wtr \& Stm } & \text { Froth } \\ 285 & 4 & \text { Reverse } & \text { Wtr \& Stm } & \text { Froth } \\ 286 & 4 & \text { Reverse } & \text { Wtr \& Stm } & \text { Slug } \\ 287 & 4 & \text { Reverse } & \text { Wtr \& Stm } & \text { Ann. } \\ 288 & 4 & \text { Reverse } & \text { Wtr \& Stm } & \text { Ann. } \\ 289 & 4 & \text { Reverse } & \text { Wtr \& Stm } & \text { Slug } \\ 290 & 4 & \text { Reverse } & \text { Wtr \& Stm } & \text { Froth } \\ 291 & 4 & \text { Reverse } & \text { Wtr \& Stm } & \text { Ann. } \\ 292 & 4 & \text { Reverse } & \text { Wtr \& Stm } & \text { Ann. } \\ 293 & 4 & \text { Reverse } & \text { Steam } & 1 \emptyset \\ 298 & 4 & \text { Reverse } & \text { Wtr \& Stm } & \text { Froth } \\ 299 & 4 & \text { Reverse } & \text { Wtr \& Stm } & \text { Froth } \\ 300 & 4 & \text { Reverse } & \text { Steam } & 10\end{array}$


transducer forces) and differential pressure dray to inomentulli flux. These calibrations were repeated at the wyle Laboratories test facility. The results from these tests provided the calibration tables for all the steam flow and two-phase flow calculations.

\section{Densitometer Beam Average Density}

Calibration tests using the three-bean densitometer were conducted at the Wyle Laboratories test facility. The calibrations involved obtaining voltage readings at known densities of water and steam. By using data collected from at least two calibration points, the constants $C$ and $D$ in equation (1) can be calculated. To ensure accurate values for these two sensitive constants, voltage readings for each calibration test need to be taken many times and then averaged. Also, in addition to the points at the extremes of the beam operating range, two or three calibration points within the range are necessary to check and modify the constants in order to obtain a proper fit to the data.

\section{SINGLE-PHASE WATER TESTS}

The three PKL spools were tested under varying all-water flow rates. Test results including comparisons to the Wyle Laboratories test facility flow rates are given in Table 5. The PKL results include an average velocity determined from the flow turbine, and an average momentum flux value determined from the three drag screen transducers. In each case, the calibration curves were obtained from tests conducted at Flow Technology, Inc. using a ballistic calibrator. The differential pressure drop across the drag screen and the calculations of percent error between the Wyle Laboratories and PKL results are also given in Table 5 .

The purpose of these water tests was primarily to provide a means of verifying the satisfactory operation of the turbine and drag screen instruments and also to use the data as an alternate means of obtaining calibration data. The allowable tolerance on velocity measurements is $+0.6 \mathrm{~m} / \mathrm{s}$; at $2.69 \mathrm{~m} / \mathrm{s}$, this tolerance is $22 \%$ of the reading. The test results show that the accuracy of the measurements generally meets this requirement. 
TABLE 5. PKL prototype spool water-flow test results.

\begin{tabular}{|c|c|c|c|c|c|c|c|c|}
\hline \multirow[b]{2}{*}{$\begin{array}{l}\text { Test } \\
\text { no. }\end{array}$} & \multirow[b]{2}{*}{$\begin{array}{c}\text { Spool } \\
\text { no. }\end{array}$} & \multicolumn{3}{|c|}{ Velocity, $\mathrm{m} / \mathrm{s}$} & \multicolumn{3}{|c|}{ Momentum flux, $\mathrm{kg} / \mathrm{m}-\mathrm{s}^{2}$} & \multirow{2}{*}{$\begin{array}{c}\Delta \text { Pres- } \\
\text { sure, } \\
\mathrm{kPa}\end{array}$} \\
\hline & & Wyle & $\begin{array}{c}\text { PKL } \\
\text { turbine }\end{array}$ & $\%$ Diff. & Wyle & PKL & $\%$ Diff. & \\
\hline 1 & 1 & 3.80 & 3.83 & 0.8 & $14,296.0$ & --- & --- & 3.08 \\
\hline 2 & 1 & 3.29 & 3.33 & 1.2 & $10,746.0$ & -- & -- & 2.26 \\
\hline 3 & 1 & 2.69 & 2.75 & 2.2 & $7,164.0$ & $7,131.0$ & -0.5 & 1.52 \\
\hline 4 & 1 & 2.43 & 2.48 & 2.1 & $5,870.0$ & $5,792.0$ & -1.3 & 1.23 \\
\hline 5 & 1 & 2.15 & 2.18 & 1.4 & $4,577.0$ & $4,507.0$ & -1.5 & 0.96 \\
\hline 6 & 1 & 1.82 & 1.85 & 1.6 & $3,283.0$ & $3,233.0$ & -1.5 & 0.68 \\
\hline 7 & 1 & 1.42 & 1.46 & 2.8 & $1,990.0$ & $1,625.0$ & -18.3 & 0.41 \\
\hline 8 & 1 & 0.834 & 0.872 & 4.6 & 691.0 & 748.0 & 8.2 & 0.14 \\
\hline 9 & 1 & 0.759 & 0.830 & 9.4 & 571.0 & 600.0 & 5.1 & 0.11 \\
\hline 10 & 1 & 0.671 & 0.693 & 3.3 & 446.0 & 428.0 & -4.0 & 0.078 \\
\hline 11 & 1 & 0.591 & 0.576 & -2.5 & 345.0 & 338.0 & -2.0 & 0.040 \\
\hline 12 & 1 & 0.461 & 0.459 & -0.4 & 113.0 & 218.0 & 92.9 & 0.018 \\
\hline 13 & 1 & 0.279 & 0.292 & 4.7 & 76.8 & 108.0 & 40.6 & -- \\
\hline 14 & 1 & 0.222 & 0.161 & -27.5 & 48.6 & 91.0 & 87.2 & --- \\
\hline 19 & 1 & -0.222 & -0.126 & -43.2 & -48.6 & -58.5 & 20.4 & --- \\
\hline 20 & 1 & -0.277 & -0.228 & -17.7 & -76.0 & -87.6 & 15.3 & -- \\
\hline 21 & 1 & -0.461 & -0.347 & -24.7 & -113.0 & -189.0 & 67.3 & --- \\
\hline 22 & 1 & -0.587 & -0.526 & -10.4 & -345.0 & -397.0 & 15.1 & -- \\
\hline 23 & 1 & -0.671 & -0.614 & -8.5 & -446.0 & -514.0 & 15.2 & -0.022 \\
\hline 24 & 1 & -0.759 & -0.735 & -3.2 & -571.0 & -647.0 & 13.3 & -0.043 \\
\hline 25 & 1 & -0.838 & -0.826 & -1.4 & -697.0 & -799.0 & 14.6 & -0.063 \\
\hline 26 & 1 & -1.42 & -1.39 & -2.1 & $-1,990.0$ & $-2,111.0$ & 6.1 & -0.22 \\
\hline 27 & 1 & -1.82 & -1.78 & -2.2 & $-3,284.0$ & $-3,451.0$ & 5.1 & -0.37 \\
\hline 28 & 1 & -2.15 & -2.11 & -1.9 & $-4,577.0$ & $-4,812.0$ & 5.1 & -0.58 \\
\hline 29 & 1 & -2.43 & -2.32 & -4.5 & $-5,871.0$ & $-6,164.0$ & 5.0 & -0.76 \\
\hline 30 & 1 & -2.69 & -2.67 & -0.7 & $-7,164.0$ & $-7,611.0$ & 6.2 & -0.92 \\
\hline 31 & 1 & -3.29 & -3.29 & 0.0 & $-10,746.0$ & --- & -- & -1.34 \\
\hline 32 & 1 & -3.80 & -3.78 & -0.5 & $-14,328.0$ & $\cdots$ & --- & -1.83 \\
\hline 103 & 3 & -0.147 & -0.133 & -11.6 & --- & --- & --- & -- \\
\hline
\end{tabular}


TABLE 5. PKL prototype spool water-flow test results. (Continued)

\begin{tabular}{|c|c|c|c|c|c|c|c|c|}
\hline \multirow[b]{2}{*}{$\begin{array}{l}\text { Test } \\
\text { no. }\end{array}$} & \multirow[b]{2}{*}{$\begin{array}{l}\text { Spool } \\
\text { no. }\end{array}$} & \multicolumn{3}{|c|}{ Velocity, $\mathrm{m} / \mathrm{s}$} & \multicolumn{3}{|c|}{ Moment um flux, $\mathrm{kg} / \mathrm{m}-\mathrm{s}^{2}$} & \multirow{2}{*}{$\begin{array}{c}\Delta \text { Pres- } \\
\text { sure, } \\
\text { kPa }\end{array}$} \\
\hline & & Wyle & $\begin{array}{c}\text { PKL } \\
\text { turbine }\end{array}$ & $\%$ Diff. & Wyle & PKL & $\%$ Diff. & \\
\hline 104 & 3 & -0.188 & -0.178 & -5.3 & $\ldots$ & -- & -- &.-- \\
\hline 105 & 3 & -0.222 & -0.207 & -6.8 & -48.9 & -122.0 & 149.0 & -- \\
\hline 106 & 3 & -0.251 & -0.296 & 17.9 & -62.8 & -149.0 & 137.0 & $\ldots$ \\
\hline 107 & 3 & -0.278 & -0.302 & 1.4 & -76.6 & -158.0 & 106.0 & --- \\
\hline 108 & 3 & -0.269 & -0.272 & 1.1 & -71.6 & -184.0 & 157.0 & -- \\
\hline 109 & 3 & -0.445 & -0.456 & 2.5 & -106.0 & -287.0 & 171.0 & --- \\
\hline 110 & 3 & -0.570 & -0.565 & -0.9 & -320.0 & -395.0 & 23.4 & --- \\
\hline 111 & 3 & -0.671 & -0.662 & -1.3 & -446.0 & -492.0 & 10.3 & --- \\
\hline 112 & 3 & -0.759 & -0.734 & -3.3 & -571.0 & -598.0 & 4.7 & -- \\
\hline 113 & 3 & -0.838 & -0.836 & -0.2 & -697.0 & -693.0 & -0.6 & --- \\
\hline 114 & 3 & -1.42 & -1.43 & 0.7 & $-1,990.0$ & $-1,902.0$ & -4.4 & -0.397 \\
\hline 115 & 3 & -1.82 & -1.83 & 0.5 & $-3,284.0$ & $-3,079.0$ & -6.2 & -0.687 \\
\hline 116 & 3 & -2.15 & -2.22 & 3.3 & $-4,577.0$ & $-4,209.0$ & -8.0 & -0.978 \\
\hline 117 & 3 & -2.43 & -2.49 & 2.4 & $-5,870.0$ & $-5,278.0$ & -10.1 & -1.24 \\
\hline 119 & 3 & -2.69 & -2.77 & 3.0 & $-7,164.0$ & $-6,661.0$ & -7.0 & -1.66 \\
\hline 120 & 3 & -3.29 & -3.35 & 1.9 & $-10,746.0$ & --- & --- & -2.43 \\
\hline 121 & 3 & -3.80 & -3.86 & 1.6 & $-14,328.0$ & $\ldots$ & --- & -3.27 \\
\hline 124 & 3 & 0.088 & 0.072 & -18.2 & 7.6 & --- & -- & --- \\
\hline 125 & 3 & 0.147 & 0.132 & -10.2 & 21.2 & $\cdots$ & --- & --- \\
\hline 126 & 3 & 0.188 & 0.179 & -5.9 & 35.1 & --- & --- & --- \\
\hline 127 & 3 & 0.222 & 0.226 & 1.7 & 48.9 & --- & -- & --- \\
\hline 128 & 3 & 0.251 & 0.256 & 2.0 & 62.8 & --- & --- & -- \\
\hline 130 & 3 & 0.269 & 0.268 & -0.4 & 72.0 & --- & --- & $\cdots$ \\
\hline 131 & 3 & 0.445 & 0.451 & 1.3 & 106.0 & 146.0 & 37.7 & --- \\
\hline 132 & 3 & 0.570 & 0.590 & 3.5 & 322.0 & 263.0 & -18.3 & 0.057 \\
\hline 133 & 3 & 0.671 & 0.664 & -1.0 & 448.0 & 386.0 & -13.8 & 0.085 \\
\hline 134 & 3 & 0.759 & 0.775 & 2.1 & 574.0 & 514.0 & -10.5 & 0.114 \\
\hline 135 & 3 & 0.838 & 0.827 & -1.3 & 697.0 & 652.0 & -0.5 & 0.146 \\
\hline 136 & 3 & 1.42 & 1.38 & -2.8 & $1,990.0$ & $1,984.0$ & -0.3 & 0.47 \\
\hline
\end{tabular}


TABLE 5. PKL prototype spool water-flow test results. (Continued)

\begin{tabular}{|c|c|c|c|c|c|c|c|c|c|}
\hline \multirow[b]{2}{*}{$\begin{array}{l}\text { Test } \\
\text { no. }\end{array}$} & \multirow[b]{2}{*}{$\begin{array}{l}\text { Spool } \\
\text { no. }\end{array}$} & \multicolumn{4}{|c|}{ Velocity, mis } & \multicolumn{3}{|c|}{ Momentum flux, $\mathrm{kg} / \mathrm{m}-\mathrm{s}^{2}$} & \multirow{2}{*}{$\begin{array}{c}\triangle \text { Pres- } \\
\text { sure, } \\
\mathrm{kPa}\end{array}$} \\
\hline & & Wyle & $\begin{array}{c}\text { PKL } \\
\text { turb ine }\end{array}$ & & $\%$ Diff. & Wyle & $\mathrm{PKL}$ & $\%$ Diff. & \\
\hline 137 & 3 & 1.82 & 1.79 & & -1.6 & $3,284.0$ & $3,195.0$ & -2.7 & 0.80 \\
\hline 138 & 3 & 2.15 & 2.24 & & 4.2 & $4,577.0$ & $4,844.0$ & 5.8 & 1.32 \\
\hline 139 & 3 & 2.43 & 2.51 & & 3.3 & $5,870.0$ & $6,090.0$ & 3.8 & 1.66 \\
\hline 140 & 3 & 2.69 & 2.75 & & 2.2 & $7,164.0$ & $7,217.0$ & 0.7 & 1.98 \\
\hline 141 & 3 & 3.29 & 3.38 & & 3.3 & $10,746.0$ & -- & --- & 3.03 \\
\hline 142 & 3 & 3.80 & 3.87 & & 1.8 & $14,328.0$ & -- & -- & 4.07 \\
\hline 233 & 4 & 3.80 & 3.74 & & -1.6 & $14,328.0$ & -- & -- & 2.74 \\
\hline 234 & 4 & 3.29 & 3.24 & & -1.5 & $10,746.0$ &.-- & --- & 2.05 \\
\hline 235 & 4 & 2.69 & 2.65 & & -1.5 & $7,164.0$ & $7,159.0$ & -0.1 & 1.36 \\
\hline 236 & 4 & 2.43 & 2.38 & & -2.1 & $5,870.0$ & $5,810.0$ & -1.0 & 1.12 \\
\hline 237 & 4 & 2.15 & 2.10 & & -2.3 & $4,577.0$ & $4,510.0$ & -1.5 & 0.87 \\
\hline 238 & 4 & 1.82 & 1.78 & & -2.2 & $3,284.0$ & $3,295.0$ & 0.3 & 0.63 \\
\hline 239 & 4 & 1.42 & 1.38 & - & -2.8 & $1,990.0$ & $2,006.0$ & 0.8 & 0.38 \\
\hline 240 & 4 & 0.838 & 0.811 & & -3.2 & 697.0 & 766.0 & 9.9 & 0.12 \\
\hline 241 & 4 & 0.570 & 0.550 & & -3.5 & 320.0 & 398.0 & 24.4 & 0.055 \\
\hline 243 & 4 & 0.278 & 0.261 & & -6.1 & 76.6 & 143.0 & 86.0 & -- \\
\hline 244 & 4 & 0.147 & 0.108 & & -26.5 & 21.2 & 76.0 & 256.0 & -- \\
\hline 267 & 4 & -3.80 & -3.81 & & 0.3 & $-14,328.0$ & -- & ..- & -2.39 \\
\hline 268 & 4 & -3.29 & -3.32 & & 0.9 & $-10,746.0$ & --- & --- & -1.78 \\
\hline 269 & 4 & -2.69 & -2.71 & & 0.7 & $-7,164.0$ & $-7,408.0$ & 4.4 & -1.16 \\
\hline 270 & 4 & -2.43 & -2.41 & & -0.8 & $-5,870.0$ & $-6,079.0$ & 3.6 & -0.93 \\
\hline 271 & 4 & -2.15 & -2.17 & & 0.9 & $-4,577.0$ & $-4,788.0$ & 4.6 & -0.72 \\
\hline 272 & 4 & -1.82 & -1.83 & & 0.5 & $-3,284.0$ & $-3,470.0$ & 5.7 & -0.52 \\
\hline 273 & 4 & -1.42 & -1.44 & & 1.4 & $-1,990.0$ & $-2,186.0$ & 9.8 & -0.31 \\
\hline 274 & 4 & -0.838 & -0.854 & & 1.9 & -697.0 & -854.0 & 22.5 & -0.11 \\
\hline 275 & 4 & -0.570 & -0.603 & & 5.8 & -320.0 & -457.0 & 42.8 & -- \\
\hline 277 & 4 & -0.278 & -0.320 & & 15.1 & -76.6 & -207.0 & 169.0 & -- \\
\hline 278 & 4 & -0.147 & -0.136 & & -7.5 & --- & --- & --- & --- \\
\hline
\end{tabular}


The accuracy requirements of the monentum flux measurements are $1 \%$ of the full-scale flux value of $7,200 \mathrm{~kg} / \mathrm{ml}-\mathrm{s}^{2}$. This accuracy requirement is satisfied for the three spools for all of the forward flow conditions. For reverse flow tests at full-scale monentum flux conditions, the spool-measured fluxes differ from the Wyle-deternined fluxes by $6.2 \%,-7.0 \%$, and $4.4 \%$ for spool nos. 1,3 , and 4 , respectively.

\section{SINGLE-PHASE SUPERHEATED STEAM TESTS}

Each of the three spools was tested. The test results data for the single-phase flow of superheated steam are given in Appendix $B$. The mass flow in each case was calculated by equation (21).

Upon inspection of the percent-difference results between the Wyle Laboratories test facility mass flow rates and the PKL Test Facility calculated mass flow rates, certain trends in the data appear as follows:

- The accuracy expressed in percent difference is generally good.

- For spool nos. 3 and 4 , the mass flow rates in the forward direction appear to be more accurate than those in the reverse direction.

- The PKL Test Facility mass flow rates, in general, are greater than the Wyle Laboratories test facility values.

In the calculation of mass flow rates, a certain percentage error is expected. Pressure reading accuracies and turbine velocity reading accuracies of $1 \%$ of full scale of $700 \mathrm{kPa}$ and $60 \mathrm{~m} / \mathrm{s}$, respectively, are expected. In the Wyle Laboratories test facility measurements, accuracies of the same order are expected. Based on these accuracies, errors on the order of 2 to $4 \%$ are not unreasonable, and much of the data appears to fall within this range.

Calibration tables for determining turbine velocity were obtained for air flow conditions on a ballistic calibrator. Improvements in the calibrations may be possible using a more steady-flow cal ibration facility. 
Each of the spools was tested in two-phase water and steam flow. The test results are given in Appendix C.

Inspection of the data indicates that the total mass flow rate accuracy is good. Total mass flow rates are more accurate at the higher values of momentum flux. In comparing the accuracy of the individual phase mass flow rates, the larger of the two is generally much more accurate, particularly for the slug flow regime tests.

OVERALL SPOOL HEAD LOSS

Measurements of overall spool head loss during single-phase water and steam flow tests were made for the prototype spools. A comparison is made between the calculated head loss obtained from the measured values of overall pressure drop and density, and the velocity head of the water or steam. A ratio of the head loss divided by the velocity head is made. Table 6 gives the results for these calculations.

The relationship for calculating velocity head (VH) is given by:

$$
V H=\frac{v^{2}}{2 g}
$$

where

$$
\begin{aligned}
& \mathrm{g}=\text { gravity } \\
& \mathrm{V}=\text { fluid velocity. }
\end{aligned}
$$

The relationship for calculating head loss ( $H L$ ) from the measured data is given by :

$$
H L=\frac{\Delta P}{\rho g}
$$


TABLE 6. Overall spool head loss during single-phase water and steam flow tests.

\begin{tabular}{|c|c|c|c|c|c|}
\hline $\begin{array}{l}\text { Test } \\
\text { no. }\end{array}$ & $\begin{array}{l}\text { Spool } \\
\text { no. }\end{array}$ & $\begin{array}{c}\Delta \text { Pressure, } \\
\text { kPa }\end{array}$ & $\begin{array}{l}\text { Density, } \\
\mathrm{kg} / \mathrm{m}^{3}\end{array}$ & $\begin{array}{l}\text { Velocity, } \\
\text { in/s }\end{array}$ & $\begin{array}{c}\text { No. of } \\
\text { Velocity Heads }\end{array}$ \\
\hline 9 & 1 & 0.27 & 995.0 & 0.759 & 0.94 \\
\hline 10 & 1 & 0.2 & 995.0 & 0.671 & 0.89 \\
\hline 11 & 1 & 0.15 & 995.0 & 0.591 & 0.86 \\
\hline 12 & 1 & 0.1 & 995.0 & 0.461 & 0.95 \\
\hline 13 & 1 & 0.045 & 995.0 & 0.279 & 1.16 \\
\hline 14 & 1 & 0.03 & 995.0 & 0.222 & 1.23 \\
\hline 15 & 1 & 0.02 & 995.0 & 0.148 & 1.84 \\
\hline 16 & 1 & 0.005 & 995.0 & 0.090 & 1.24 \\
\hline 18 & 1 & 0.01 & 995.0 & 0.148 & 0.92 \\
\hline 19 & 1 & 0.02 & 995.0 & 0.222 & 0.82 \\
\hline 20 & 1 & 0.03 & 995.0 & 0.277 & 0.79 \\
\hline 21 & 1 & 0.06 & 995.0 & 0.461 & 0.57 \\
\hline 22 & 1 & 0.13 & 995.0 & 0.587 & 0.76 \\
\hline 23 & 1 & 0.18 & 995.0 & 0.671 & 0.81 \\
\hline 24 & 1 & 0.23 & 995.0 & 0.759 & 0.80 \\
\hline 25 & 1 & 0.29 & 995.0 & 0.838 & 0.83 \\
\hline 26 & 1 & 0.82 & 995.0 & 1.42 & 0.82 \\
\hline 27 & 1 & 1.33 & 995.0 & 1.82 & 0.81 \\
\hline 28 & 1 & 1.85 & 995.0 & 2.15 & 0.81 \\
\hline 29 & 1 & 2.45 & 995.0 & 2.43 & 0.84 \\
\hline 30 & 1 & 2.99 & 995.0 & 2.69 & 0.83 \\
\hline 31 & 1 & 5.22 & 995.0 & 3.29 & 0.97 \\
\hline 32 & 1 & 5.90 & 995.0 & 3.80 & 0.82 \\
\hline 47 & 1 & 0.917 & 3.2 & 32.2 & 0.55 \\
\hline 48 & 1 & 2.750 & 2.2 & 56.2 & 0.79 \\
\hline 61 & 1 & 3.221 & 2.2 & 56.5 & 0.92 \\
\hline 63 & 1 & 0.11 & 2.1 & 8.78 & 1.4 \\
\hline 64 & 1 & 0.655 & 1.1 & 39.8 & 0.75 \\
\hline 71 & 1 & 0.412 & 2.2 & 17.9 & 1.2 \\
\hline 72 & 1 & 2.796 & 2.1 & 57.0 & 0.82 \\
\hline 73 & 1 & 0.052 & 2.2 & 7.05 & 0.95 \\
\hline
\end{tabular}


TABLE 6. Overall spool head loss during single-phase water and stean flow tests. (Cont inued)

\begin{tabular}{|c|c|c|c|c|c|}
\hline $\begin{array}{l}\text { Test } \\
\text { no. }\end{array}$ & $\begin{array}{l}\text { Spool } \\
\text { no. }\end{array}$ & $\underset{\mathrm{kPa}}{\Delta \text { Pressure }}$ & $\begin{array}{l}\text { Density } \\
\mathrm{kg} / \mathrm{m}\end{array}$ & $\begin{array}{l}\text { Velocity, } \\
\text { m/s }\end{array}$ & $\begin{array}{l}\text { No. of } \\
\text { Velocity Heads }\end{array}$ \\
\hline 88 & 1 & 0.637 & 1.2 & 36.8 & 0.79 \\
\hline 89 & 1 & 2.911 & 2.2 & 56.7 & 0.82 \\
\hline 90 & 1 & 0.942 & 3.2 & 28.8 & 0.71 \\
\hline 119 & 3 & 2.28 & 995.0 & 2.69 & 0.63 \\
\hline 120 & 3 & 3.22 & 995.0 & 3.29 & 0.60 \\
\hline 121 & 3 & 4.42 & 995.0 & 3.80 & 0.62 \\
\hline 125 & 3 & 0.005 & 995.0 & 0.147 & 0.47 \\
\hline 126 & 3 & 0.01 & 995.0 & 0.188 & 0.57 \\
\hline 127 & 3 & 0.012 & 995.0 & 0.222 & 0.49 \\
\hline 128 & 3 & 0.023 & 995.0 & 0.251 & 0.74 \\
\hline 130 & 3 & 0.018 & 995.0 & 0.269 & 0.50 \\
\hline 131 & 3 & 0.072 & 995.0 & 0.445 & 0.73 \\
\hline 132 & 3 & 0.120 & 995.0 & 0.570 & 0.74 \\
\hline 133 & 3 & 0.175 & 995.0 & 0.671 & 0.78 \\
\hline 134 & 3 & 0.228 & 995.0 & 0.759 & 0.80 \\
\hline 135 & 3 & 0.277 & 995.0 & 0.838 & 0.79 \\
\hline 136 & 3 & 0.687 & 995.0 & 1.42 & 0.69 \\
\hline 137 & 3 & 1.132 & 995.0 & 1.82 & 0.69 \\
\hline 138 & 3 & 2.073 & 995.0 & 2.15 & 0.91 \\
\hline 139 & 3 & 2.690 & 995.0 & 2.43 & 0.92 \\
\hline 140 & 3 & 3.230 & 995.0 & 2.69 & 0.90 \\
\hline 141 & 3 & 4.757 . & 995.0 & 3.29 & 0.89 \\
\hline 142 & 3 & 6.457 & 995.0 & 3.80 & 0.90 \\
\hline 148 & 3 & 2.540 & 2.2 & 54.40 & 0.78 \\
\hline 157 & 3 & 2.939 & 2.2 & 54.3 & 0.91 \\
\hline 172 & 3 & 3.008 & 2.2 & 55.0 & 0.91 \\
\hline 183 & 3 & 2.018 & 2.2 & 56.8 & 0.57 \\
\hline 194 & 3 & 2.300 & 2.2 & 57.2 & 0.64 \\
\hline
\end{tabular}


where

$$
\begin{aligned}
\rho & =\text { fluid density } \\
\Delta \rho & =\text { spool overall pressure drop. }
\end{aligned}
$$

Dividing equation (29) by equation (28) gives the number of velocity heads pressure 10ss. From an inspection of the test results in Table 6 , it is noted that the number of velocity heads for the majority of the tests is below a value of 1.0 .

40-CYCLE LIMITING CONDITION SURVIVAL TESTS

Spool nos. 1 and 3 were tested for 40 cycles between a zero-water flow rate and a water flow rate corresponding to a inomentum flux condition of $14,400 \mathrm{~kg} / \mathrm{m}-\mathrm{s}^{2}$. For each cycle, engineering unit data were obtained at zero-water flow and at the liniting momentum flux condition. Results of the tests show no observable degradation of perfomance of the spool instrunents. 
Three PKL instrumented prototype spools were tested in single-phase water, single-phase superheated steam, and two-phase steam ana water flows. For single-phase water flow tests, turbine velocity measurements are within the allowable tolerance limit and show good agreement with the Wyle facility measureinents. Drag screen measurements of momentum $\mathrm{flux}$ in the forward-flow direction are generally within tolerance. In the reverse-flow direction, measured momentuin flux accuracies are not as good as in the forward-flow direction ald generally exceed allowable tolerance limits.

For single-phase superheated steam flow tests, measurements of steain mass flow rates appear very good. In analyzing the accuracy of the data, it is generally expected that accuracies decrease for lower velocity flows. The accuracies for reverse-flow tests on spool no. 4 do not appear to be as good as on the other two spools tested.

In two-phase flows, the accuracy of measured total mass flow rate is good. In analyzing the accuracies of the measured flows, consideration must be given $=0$ the accuracy requirements of the instruments. In general, accuracies decrease with decreases in momentum flux and velocity. 


\section{REFERENCES}

(1) R. F. Smellie, PKL Instrumented Spool Piece Final Design Review, EG\&G, Inc., San Ramon Operations, MES-014, Tab 2, p. 35 (Apri1 1979).

(2) G. D. Lassahn, Loft Experimental Measurements Uncertainties Analyses. Volume 16: Loft Three-Beam Gamma Densitometer System, Tree-NUREG-1089 (February 1978).

(3) G. D. Lassahn, Loft Three-Beam Densitometer Data Interpretation, Tree-NUREG-1111 (0ctober 1977).

(4) Calculations developed by J. Colson, INEL, Idaho Falls, Idaho; private communication W. Stein/J. Colson (January 1978).

(5) H. Estranda, Jr., and J. D. Sheppard, Some Aspects of Interpreting Two-Phase Flow Measurements in Instrumented Piping Spool Pieces, NUREG-0280 (June 1977).

LMS/amr(EG\&G/SRO) 
APPENDIX A

PKL SPOOL PIECE DRAWINGS

(EG\&G DRAWING NOS. 137086, 137087, AND 137088) 


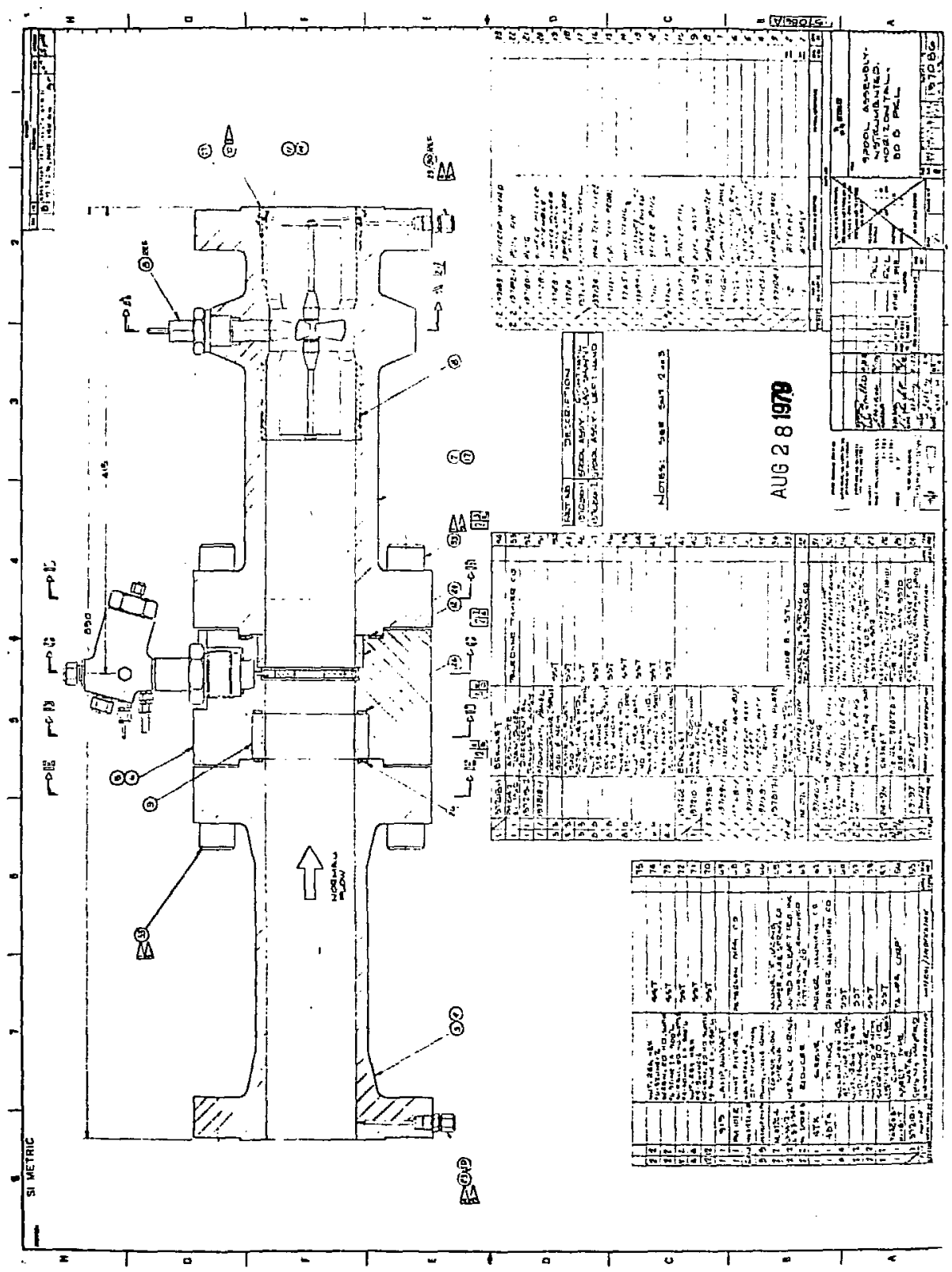




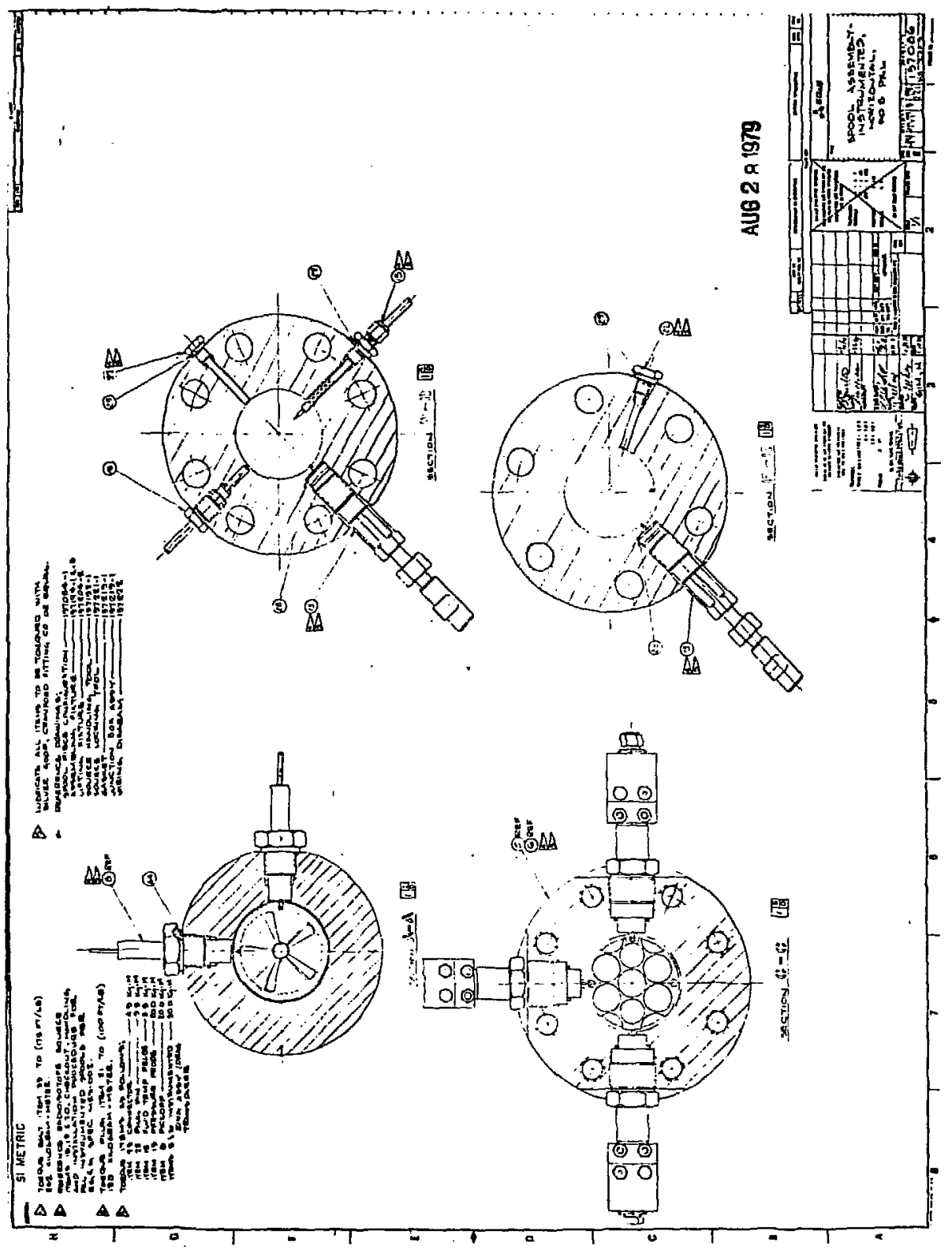




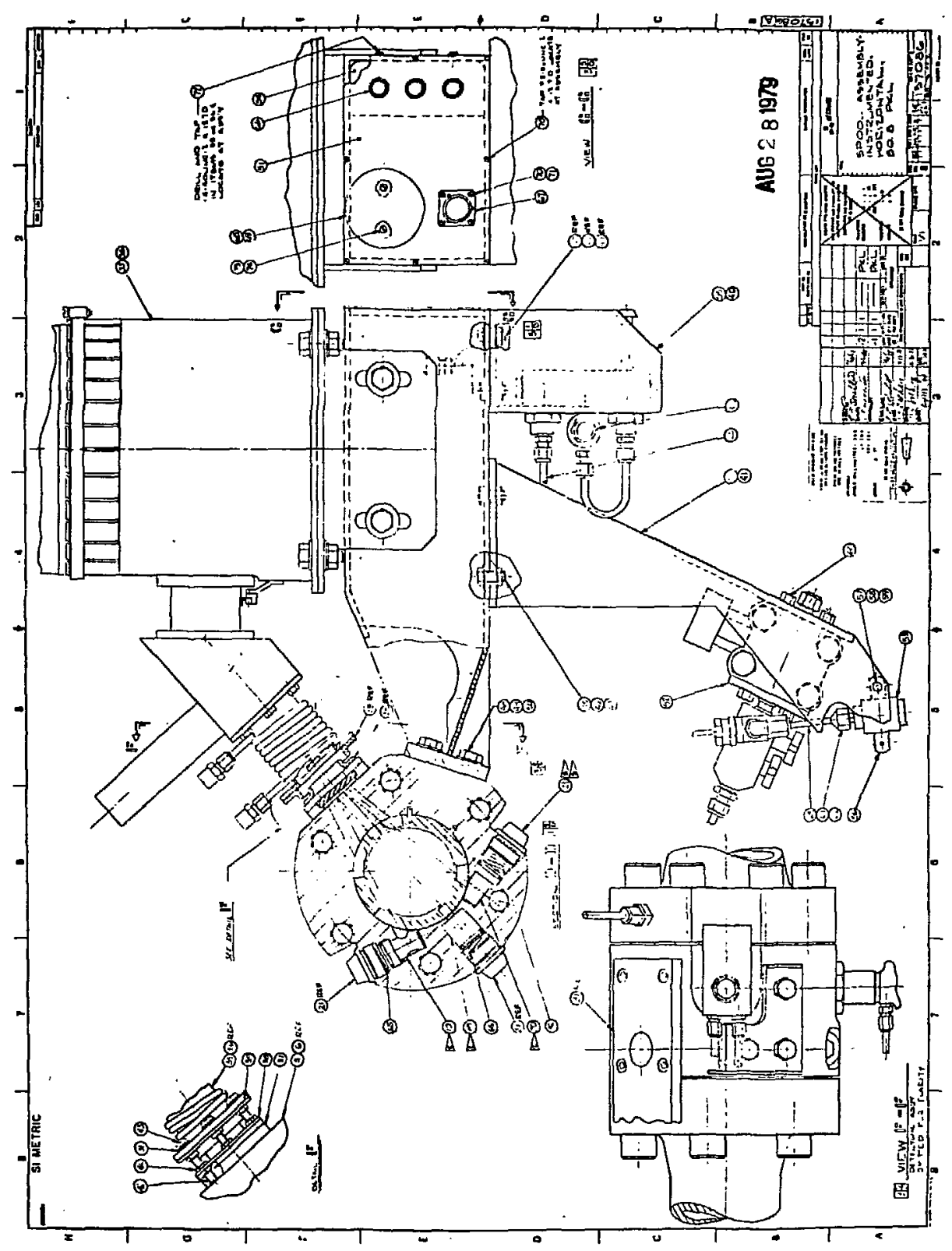




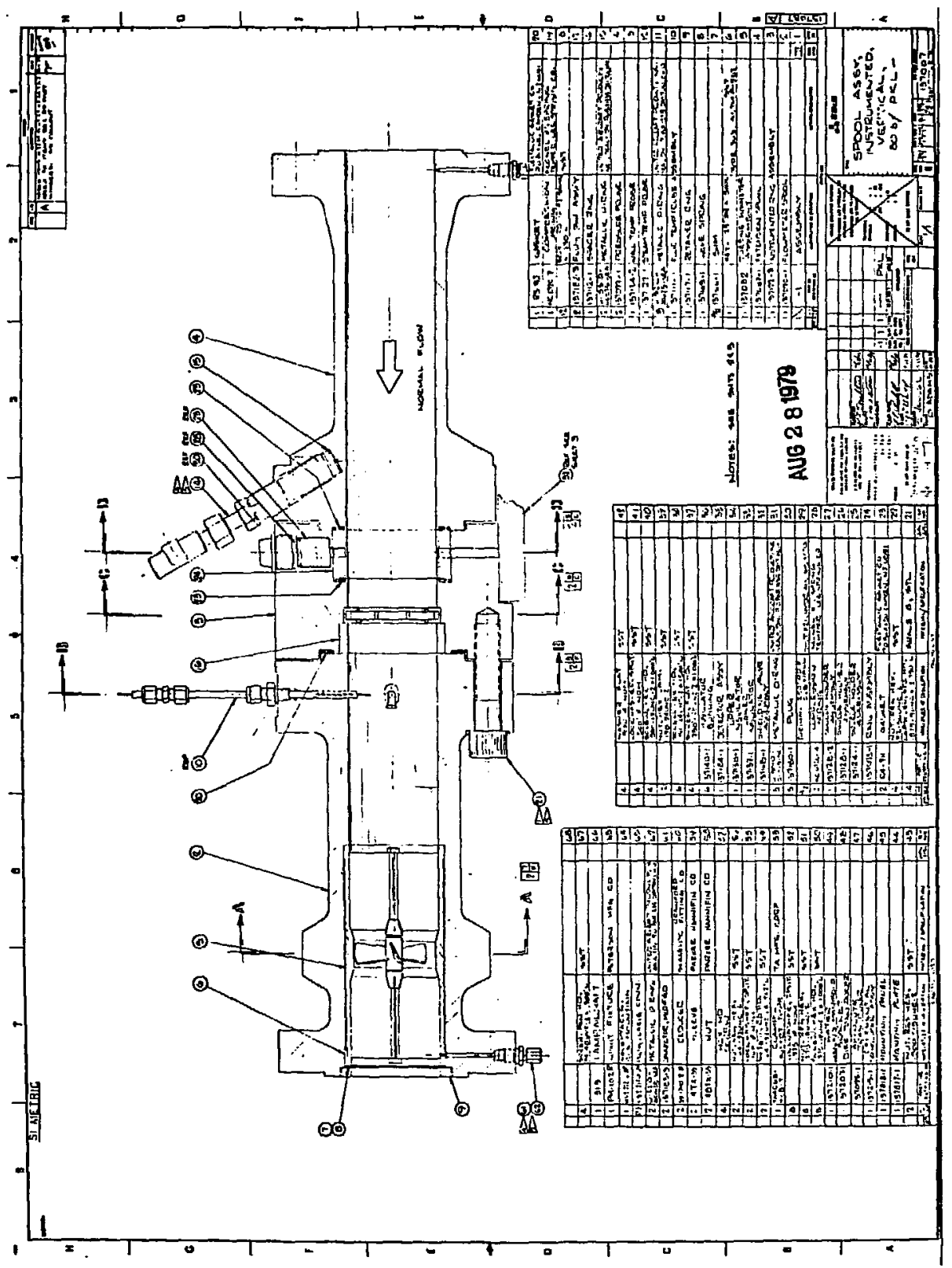




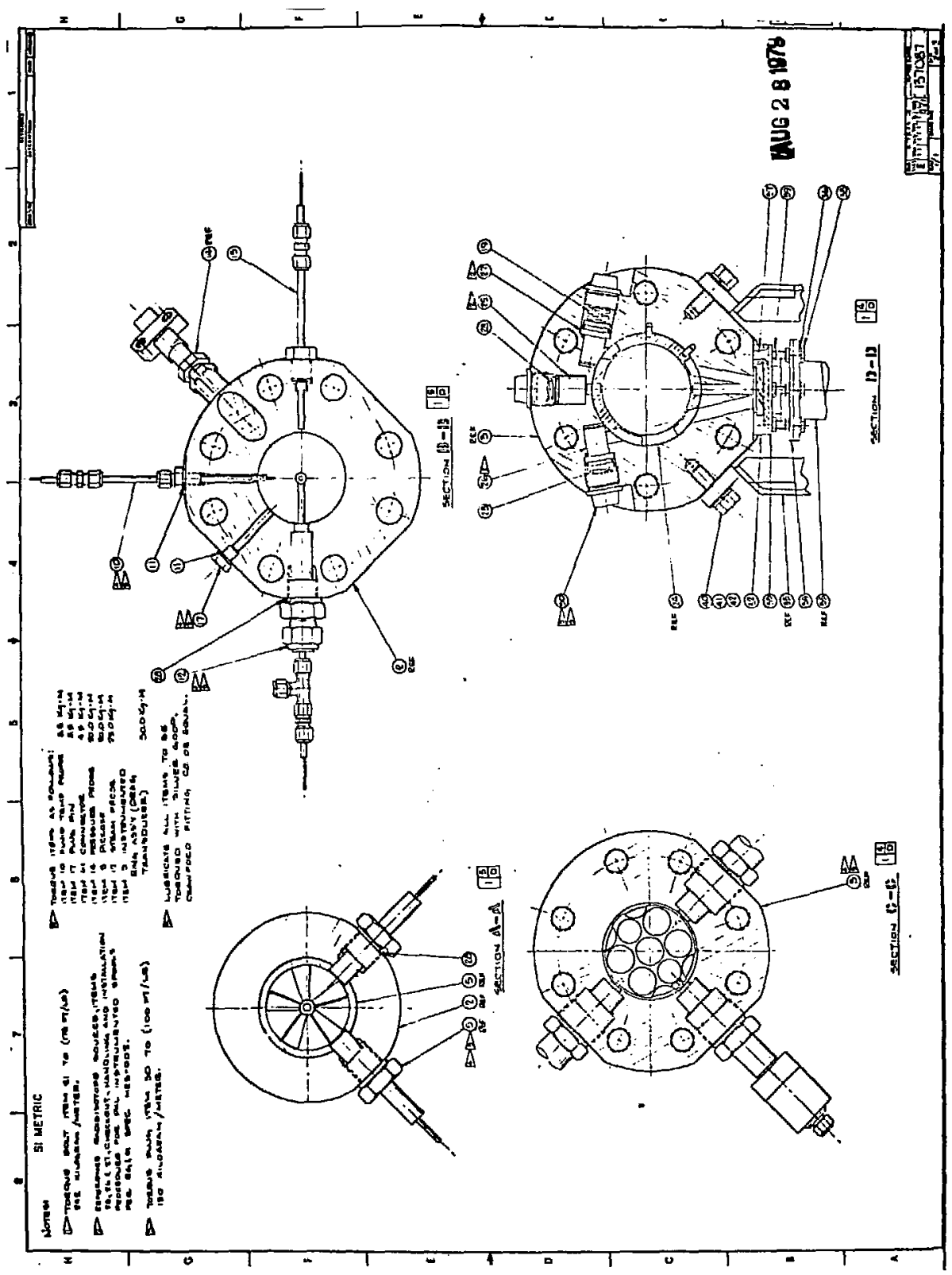




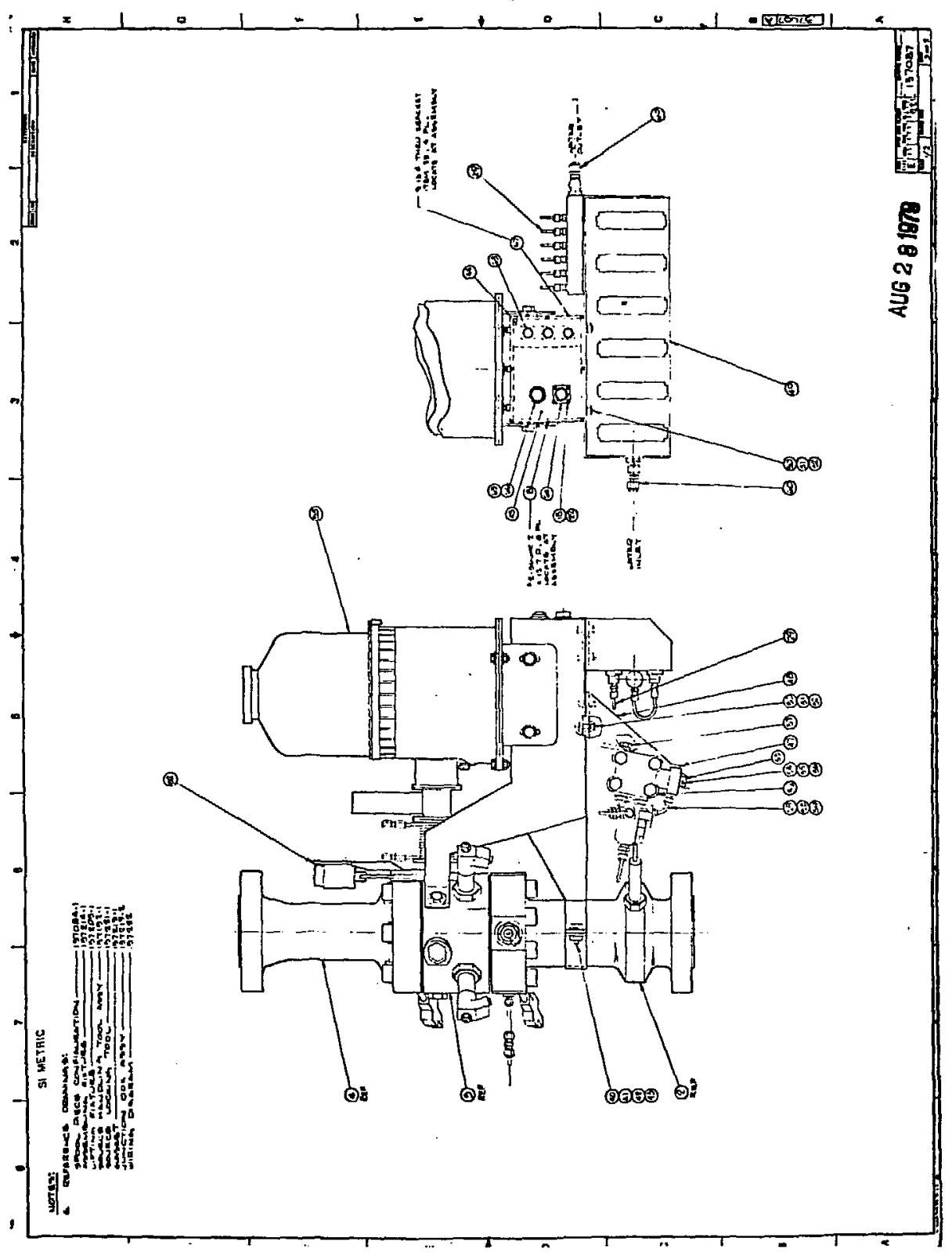




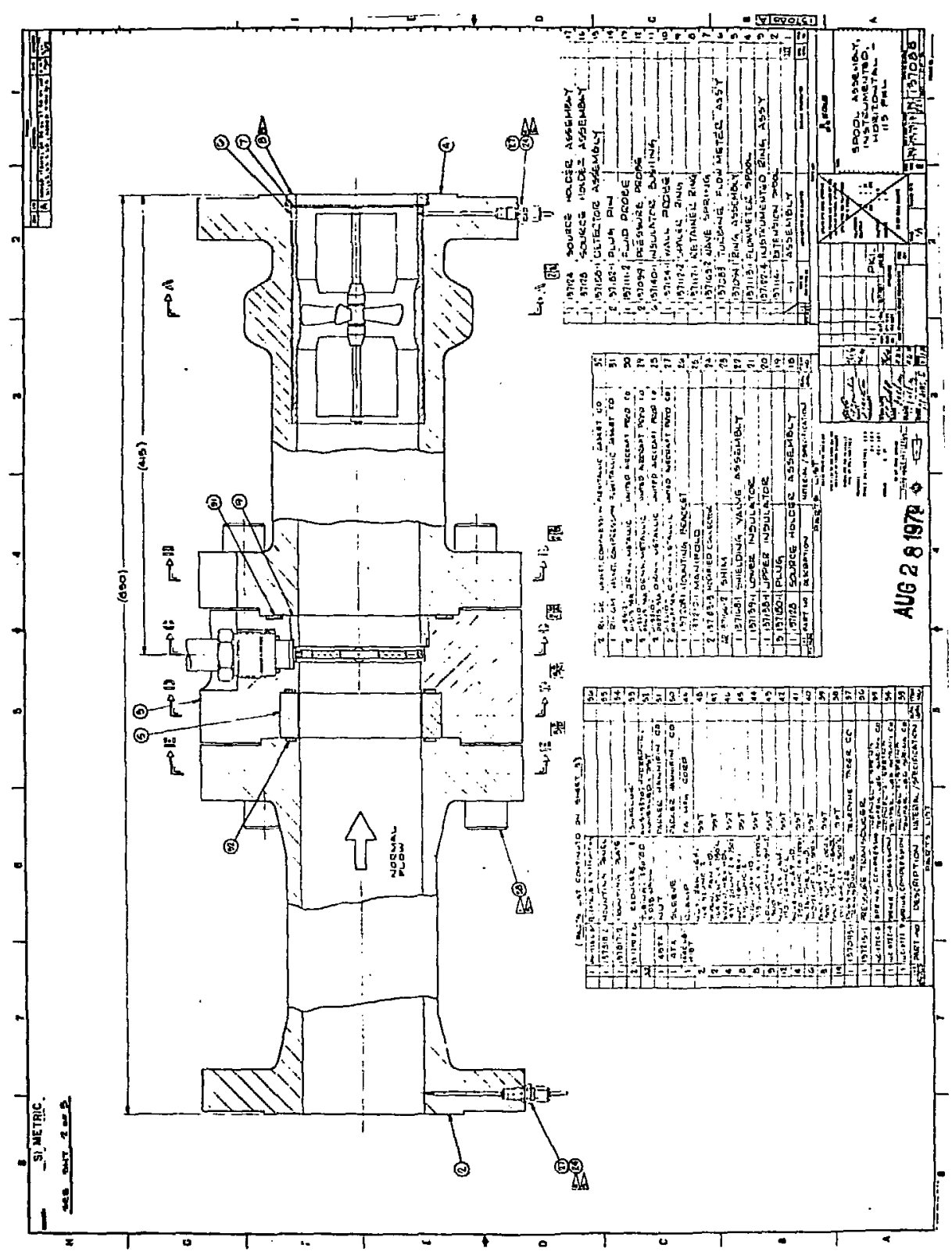




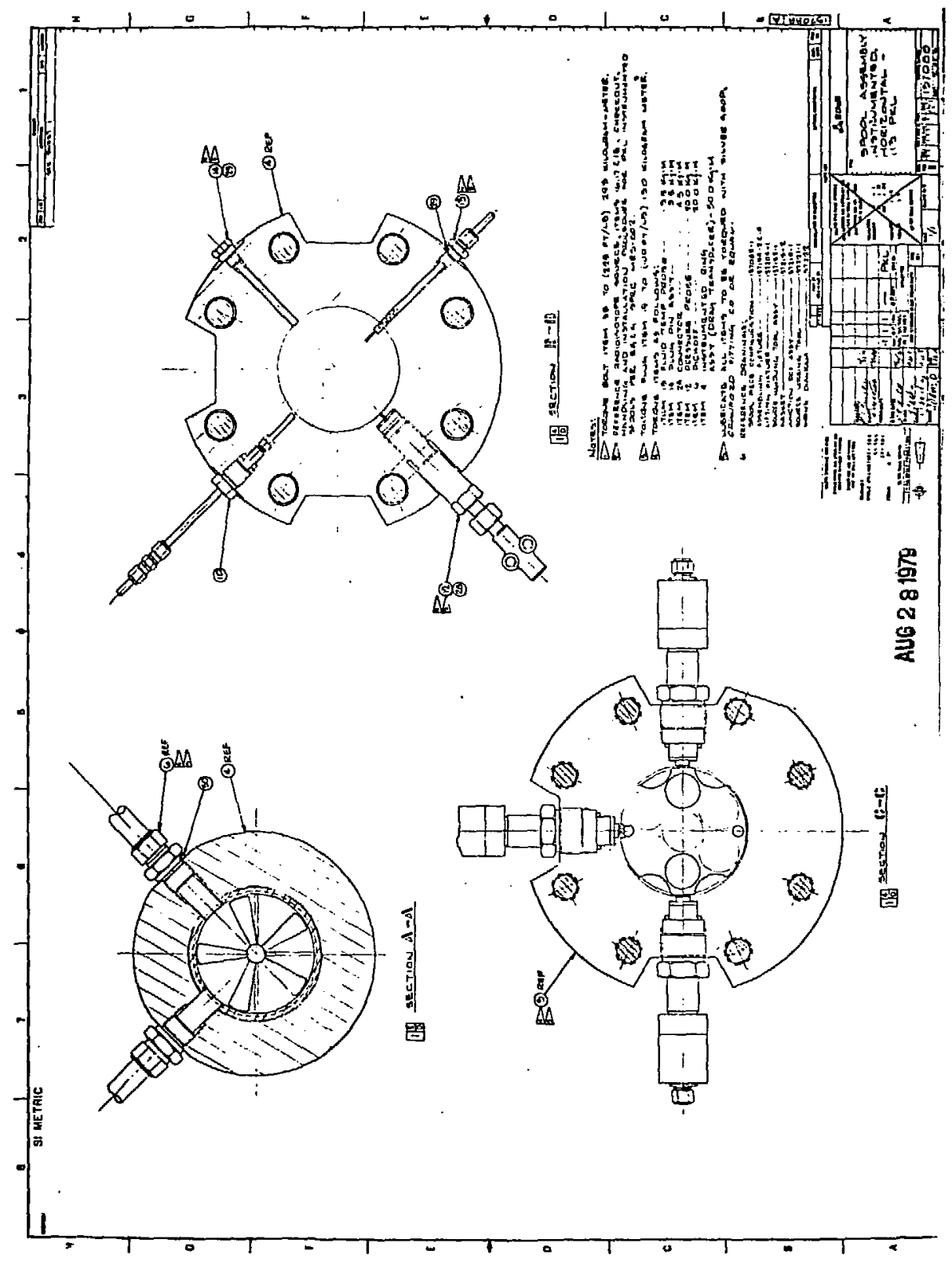




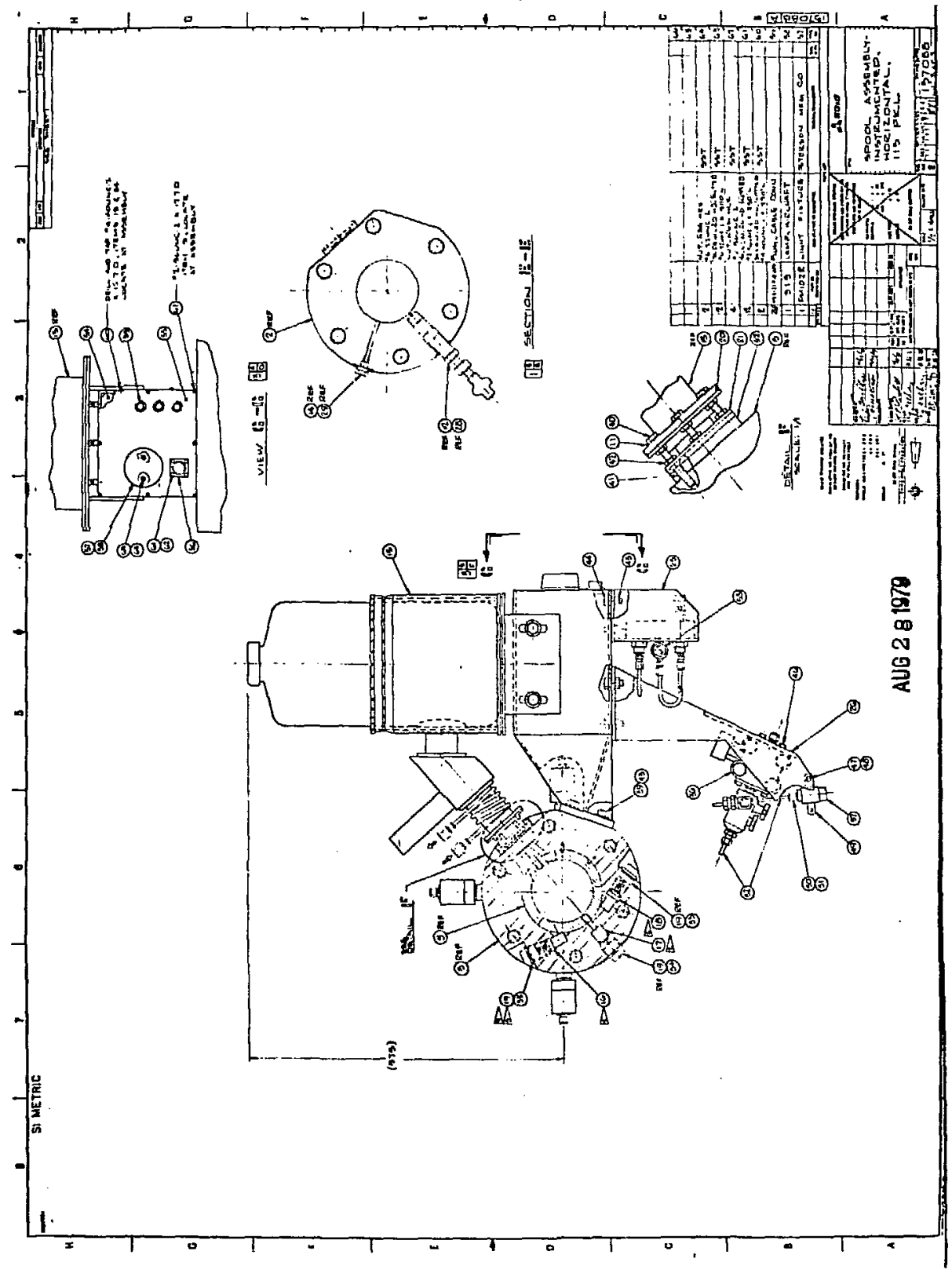


APPENDIX B

PKL SPOOL PIECE TEST RESULTS FROM SINGLE-PHASE SUPERHEATED-STEAM TESTS 


\section{APPENDIX B}

The data presented in this appendix give the test results for the single-phase superheated-steam flow tests and compare these results to the Wyle Laboratory test facility measured results.

The test numbers applicable to each of the three spools tested are given below:

Spool No.

1

3

4
Test No.

$38-97$

$\cdot 148-194$

$252-300$

A test number is indicated for each test and the data is presented with the printouts in the following format:

- First row: Average steam flow data is given to the right of the heading "STEAM FLOW =" with numerical values given in the "WYLE" AND "PKL" columns. In the same row, a percent difference calculated by equation (25) is given for these values under the col umn "\% DIFF", and a deviation calculated by equation (27) is given for steam flow rate under col umn "DEVIATION".

- Second row: Data is given on standard deviations associated with the Wyle and PKL steam flow rates, respectively, and is calculated using equation (24).

- Third and fourth rows: Similar data as for the above two rows is presented for water flows. 
- Fifth row: Data is given on the total flow which is a sum of the steani and water flow rates already given. A percent difference between the total Wyle and PKL flow is given in the "\% DIFF" column and is calculated by equation (25).

- To the right of the above data, under the general heading of "AVERAGE PKL TEST PARAMETERS", are given the average values for the engineering unit data obtained from each of the instrument channels. Identification for each of the abbreviations is given below:

TF

= fluid temperature.

TW = wall temperature.

TS = superheated-steam temperature (applicable only to spool

no. 4).

$\mathrm{DF1}, \mathrm{DF} 2, \mathrm{DF} 3$ = drag screen transducer force for transducer nos. 1, 2, and 3.

$\mathrm{GD1}, \mathrm{GD2}, \mathrm{GD} 3$ = density along beam nos. 1,2 , and 3 of the densitomettr.

PA $\quad$ fluid absolute pressure.

PD $\quad=$ drag screen differential pressure.

FT = turbine velocity.

DTT = drag screen momenturi flux. 
FKL PROTOTYPE SFOQL TEST RESULTS TEST NO. UNITS (KG/S) WYLE Pk:

39

$\begin{array}{rlll}\text { STEAM FLOW }= & 0.604 & 0.614 & 1.68 \\ \text { STD DEV }= & 0.001 & 0.003 & \\ \text { WATER FLOH }= & 0.000 & 0.000 & 0.00 \\ \text { STD DEV }= & 0.000 & 0.000 & \\ \text { TOTAL FLOW }= & 0.604 & 0.614 & 1.68\end{array}$

PKL PROTOTYPE SPOOL TEST RESULTS TEST NO. UNITS (KG/S) WYLE

PKL. Z IIIFF DEVIATION

47

$\begin{array}{rlr}\text { STEAM FLOW } & =0.490 & 0.530 \\ \text { STD DEV } & =0.003 & 0.005 \\ \text { WATER FLOW } & =0.000 & 0.000 \\ \text { STD DEV } & =0.000 & 0.000 \\ \text { TOTAL FLOW } & =0.490 & 0.530\end{array}$

a. 22

0.0413

DEG C AVERAGE FILL TEST PAFIMETEFO
$T W=150.1[$ [IF $2=3.201$ (ODZ $=2.7 \quad \mathrm{FT}(\mathrm{M} / \mathrm{S})=56.47$

$T S=0.0 \mathrm{DF} 3=0.019$ COD $3=$

$2.0 \operatorname{UTT}(\mathrm{KT} / \mathrm{M}-\mathrm{S})=6567.2$

AVERAGE FKL. TEST FARAIJETEFE:

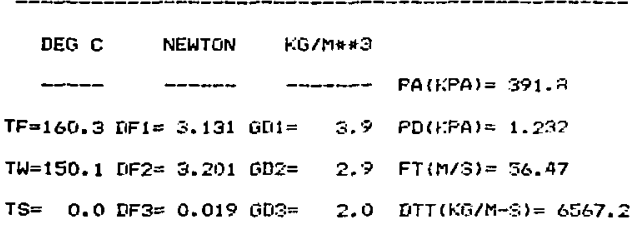

PKL PROTOTYPE SPOOL TEST RESULTS

PKL PROTOTYPE SPOOL TEST RESULT
TEST NO. UNITS (kG/S) WYLE

48

$\begin{array}{rllll}\text { STEAM FLOW } & =0.607 & 0.632 & 4.19 & 0.0260 \\ \text { STD DEV } & =0.002 & 0.002 & & \\ \text { HATER FLOW } & =0.000 & 0.000 & 0.00 & 0.0000 \\ \text { STD LEV } & =0.000 & 0.000 & & \\ \text { TOTAL FLOW } & =0.607 & 0.632 & 4.19 & \end{array}$

AVERALIE PHLL TEST FARAMETERS

LEG C: NEWTON H:O/NH

-m-D - - - -

$T F=160.3 D F 1=3.285 \quad B D 1=4.2 \quad F[1(t: F A)=1.310$

$T W=153.0 \mathrm{DF} 2=3.375 \mathrm{GCL}=3.1 \mathrm{FT}(\mathrm{M} / \mathrm{S})=56.24$

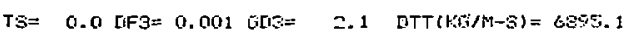


FhL PROTUTYFE SPOOL TEST RESILTS TEST NO. ININIS (K,G/S) WYLE FKL

61 STEAM FLOW $=$
STD DEV $=$

0.609

0.626

2.75

0.0173

0.002

WATEF FLOW =

0.000

0.003

STD DEV =

0. ono

0.000

0.00

0.0000

0.000

TUTAL FLOW =

0.609

0.626

2.75

FKL PROTOTYFE SPOOL TEST RESLILTS TEST NG. UNITS (K.G/S) WYLE

FHL \% IIFF DEVIATION

62

0.205

$0.20=$

$-1.01$

0.0033

0.003

0.000

0.000

0.00

0.0000

STD $\mathrm{DEV}=$

0.000

0.000

TOTAL FLOW $=$

$0.203 \quad-1.01$

$$
\begin{aligned}
\text { STEAM FLCIW } & = \\
\text { STL IEV } & =
\end{aligned}
$$

0. 001

WATEK FLOW: =

AVERAGE FKL TEST FARAMETERS

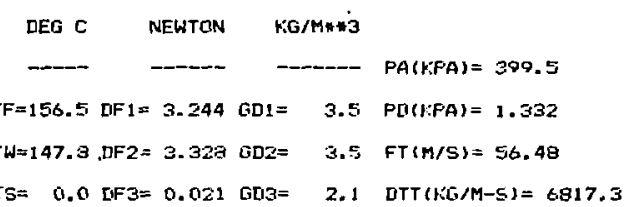

AVERACE PKL TEST PARAMETEKS

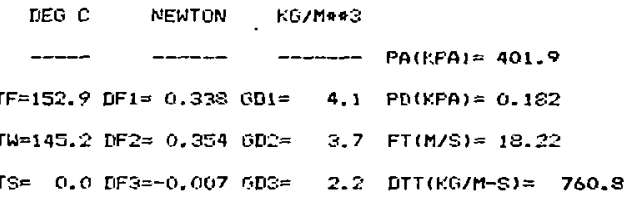

FRL PRGTOTYPE SFOUL TEST RESILTS TEST NO. UNJTS (WOO/S) WYLE FH

63 STEAM FLOW $=$
STI LEV $=$
WATER FLOW $=$
STU LEV $=$
TETA! FLOW $=$

0.097

$0.095 \quad-2.23$

0.0022

0.003

D. 003

0.000

0.000

0.00

0.0000

0.000

0.097

$0.055 \quad-2.22$

DEVIATION

\section{.}

DEG C NENTON KERM\#

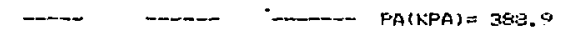

$T F=149.4[F 1=0.081 \quad[B D 1=6.9 \quad F[(K F A)=0.067$

$T W=143.9$ NF2 $=0.087$ GDZ $=3.0$ FT(M/S) $=3.78$

$T S=0.0$ [FF $3=-0.002$ CLSS 2.9 DTT $(1.0 / M-S)=205.4$ 
FKL FROTOTYPE SFONL TEST FESIILTS TEST NO. UNITS (KIS/S) WYLE PKLL \% DIFF DEVIATION -

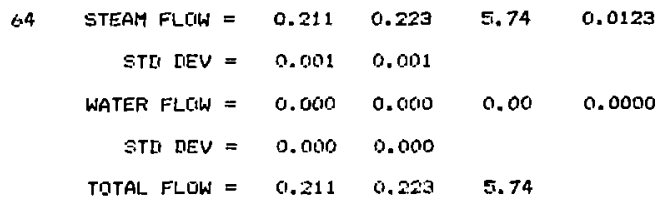

FHLL PROTOTYPE SPODL TEST FEEIJLTS TEST NO. UNITS $(k, T / S)$ WYLE.

PHL \% LIFF DEVIATIIN

\section{1}

$\begin{array}{rlrl}\text { STEAM FLOW } & =-0.204 & -0.204 & 0.23 \\ \text { STD DEV } & =0.001 & 0.002 & \\ \text { WATER FLOW } & =0.000 & 0.000 & 0.00 \\ \text { STD DEV } & =0.000 & 0.000 & \\ \text { TUTAL. FLOW } & =-0.204 & -0.204 & 0.23\end{array}$

0.0022

0.0000

$\mathrm{TE}=15 \mathrm{i3} .2 \mathrm{BF} 1=-0.335 \mathrm{BDt}=$ $T W=143.6$ DF2 $=-0.355$ 5D2=

$T S=0.0$ rif $3=-0.022$ ODS $=$

AVERAISE PKL TEST PARAMETERS

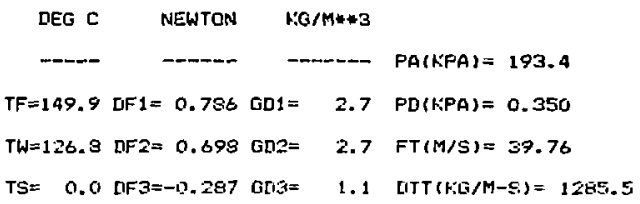

FKL FROTOTYPE GFOUL TEST RESIILTS TEST NO. UNITS (H:G $/ S$ ) WYLE FKL \% DIFF TIEVIATION

$\begin{array}{rlrll}\text { STEAM FLOW } & =-0.600 & -0.620 & 0.02 & 0.0195 \\ \text { STD IEW } & =0.002 & 0.006 & & \\ \text { WATER FLOW } & =0.000 & 0.000 & 0.00 & 0.0000 \\ \text { STD DEV } & =0.000 & 0.000 & & \\ \text { TOTAL FLOW } & =-0.608 & -0.620 & 3.02 & \end{array}$

AUFRALE FYLL TEST PARAMETEKS

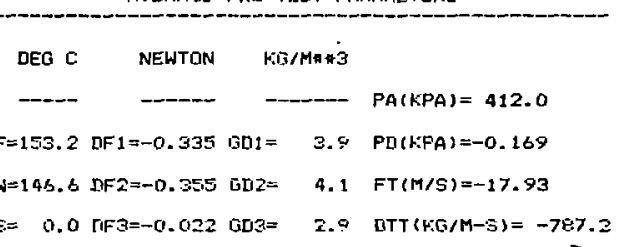

AVERAISE F'KLL TEST FARAMEIERS:

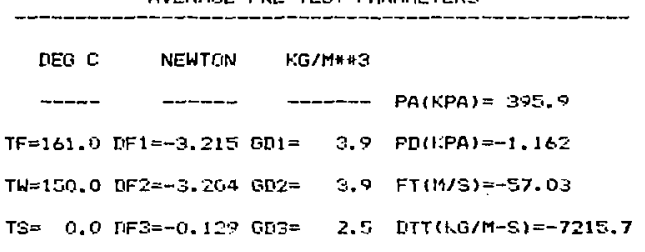


PKL PROTOTYPE SFUIL TEST HESIN.TS

TEST NO. UNITS (KE/S) WYLE FKL \% DIFF DEVIATION

73

$\begin{array}{rlrr}\text { STEAM FLOW } & =-0.102 & -0.078 & -23.17 \\ \text { STI LIEV } & =0.002 & 0.001 & \\ \text { WATER FLOW } & =0.000 & 0.000 & 0.00 \\ \text { STD NEV } & =0.000 & 0.000 & \\ \text { TOTAL FLOW } & =-0.102 & -0.079 & -2.3 .17\end{array}$

0.0240

VIES C TOTAL, FLOW $=-0.102-0.079-23.17$

PFLL PROTOTYFE SFOUL TEST FESI ILTS

TEST NO. LINITS (KL̈/S)
WYLE PKL Z UIFF DEVIATION

0.0000 - - - - - NG/MH*3

$T F=150.0$ OF $1=-0.05 A$ CD1 $=34.1 \quad$ PD(YFA $)=-0.010$ $\mathrm{TW}=145.4 \mathrm{nF} 2=-0.079$ GN $2=4.2 \mathrm{FT}(\mathrm{M} / \mathrm{S})=-7.05$ $T S=0.0$ [15 $S=-0.015$ GDS:= 3.4 DTT $(K G / M-\varepsilon)=-206.1$
STEAM FLOW $=-0.212-0.221$
0.003
0.002
WATEK FLOW $=0.000$
0.000
STR NEV $=0.1000$
0.000
TOTAL, FLOH $=-0.212-0.221$
4.25

0.0059

DEE $C$

- $-\cdots$

0.0000

$T F=135.9 \quad 0 F 1=-0.725601=$

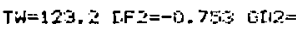
TS: $0.0 \mathrm{DFS}=-0.045$ 1015-
AUERAGE FKL TE:T FARAMETEKS

NEWTON $1: G / 17 \%$ W

-OP PA(KPA) $=207.8$

$3.4 \quad F(1): F A)=-0.245$

4.3 Frim, $5=-3.37$

1.1 पT (1:G/N-S: $=-1722.7$

PKL FRDTOTYFE GFOOL TEST FFSLUTS

TEST HO. UNITS (KG/S) WYLE

PK'L \% IIIFF IIEVIATIIN

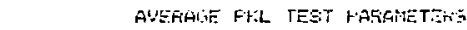

89

$\begin{array}{rlrlr}\text { STEAM FLOW } & =-0.610 & -0.640 & 4.89 & 0.0304 \\ \text { STII DEV } & =0.002 & 0.002 & & \\ \text { WATER FLOH } & =0.000 & 0.000 & 0.00 & 0.6000 \\ \text { STI IEU } & =0.000 & 0.000 & & \\ \text { TOTAL FLOW } & =-0.690 & -0.640 & 4.69 & \end{array}$

LES 6. NEWTIN HBMAES

-

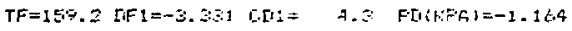

$T W=14.5 .1$ T.F $2=-3.321 \quad \mathrm{DN}=4.6 \quad \mathrm{Fr}(\mathrm{H}, \mathrm{S})=-5 \mathrm{t} .73$

$T S=0.0[1 / 23=-1) .0134$ ans $3=$

2.0 [.TT $\left(x, 1, M_{1}-5\right)=-75070.2$ 
PKL PROTOTYFE SFOOL TEST RESULTS

TEST NO. UNITS (KG/S)

$$
\text { WYLE }
$$

PKL

Z DIFF IIEUIATION

$\$ 0$

3.34

0.0163
STD DEV =

0.002

0.005

WATER FLOW $=0.000$

0.000

0.00

0.0000

STD DEV $=0.000$

0.000

TOTAL FLOW $=-0.462-0.477$

3.34
AVERAGGE FKI TEET FGARAMII:TERS

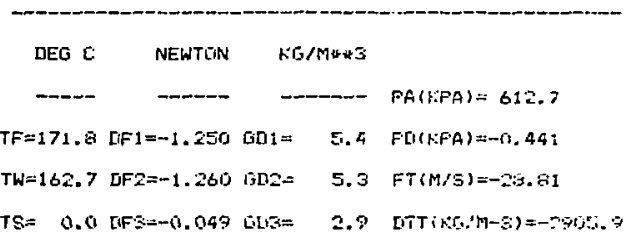

FHL FEOTOTYFE SFGOL TEST AESIM_TE

$\begin{array}{rlrlll}\text { TEST NO. UNITS (KIS/S) } & \text { WYLE } & \text { FKL } & \text { \% DIFF } & \text { DEVIATION } \\ 97 \text { STEAM FLOW } & =-0.007 & -0.619 & 1.82 & 0.0114 \\ \text { STL DEV } & =0.001 & 0.002 & & \\ \text { WATER FLOW } & =0.000 & 0.000 & 0.00 & 0.0000 \\ \text { STO DEV } & =0.000 & 0.0100 & & \\ \text { TOTAL FLOW } & =-0.007 & -0.619 & 1.52 & \end{array}$

FH'L PROTOTYPE APOLL TEST RESIJLTS

TEST ND. UNITS (KG/S) WV'LE FK AVERAGE PKL TEST PARAMETERS

\begin{tabular}{|c|c|c|c|c|}
\hline DEG $C$ & NEWTON & & $4 * 43$ & \\
\hline$-\infty$ & $-\infty$ & & -- & $P A(K F A)=405.7$ \\
\hline$=161.5$ & $\mathrm{DF} 1=-3.097$ & $G D 1=$ & 3.8 & $P\left[\left(1^{\prime} P A\right)=-1.137\right.$ \\
\hline$y=147.3$ & $\mathrm{NF}:=-3.05 .3$ & $602=$ & 4.7 & $\mathrm{FT}(\mathrm{m} / \mathrm{s})=-54.99$ \\
\hline
\end{tabular}

148

$$
\text { ST }
$$

1.12E 1.191

4.65

DIEVIATION

AVERAGE FKL TEST PARAMETEKS

$$
\text { STU DEV }=0.007 \quad 0.004
$$

WATER FLOW $=0.000 .000$

0.00

0.1000

0.0537

DEG C

NEWTON

NOS/M**3

$-\cdots$

…--

- PA(KPA $)=404.1$

GTD NEV $=0.000 \quad 0.000$

TOTAL FLCW =

1. 13 ?

1. 191

4.65

$T F=162.7 \quad[F 1=6.182$ GD $1=$

$0.0 F D(1: P A)=2.193$

$T W=144.1 \mathrm{DF} 2=6.278 \mathrm{GD} 2=$

$0.0 \mathrm{FT}(\mathrm{M} / \mathrm{S})=\mathbf{5 4 . 3 \mathrm { a }}$

$T S=$

$0.0 \mathrm{DF} 3=0.269 \mathrm{nD} 3=1.2 \mathrm{DTT}(\mathrm{KG} / \mathrm{M}-\mathrm{S})=6273.0$ 
PKL PROTOTYPE SPQOL TEST RESULTS TEST NÜ. UNITS (K'G/S) WYLE PKL * DIFF DEVIATION AVERAGE PRL TEST PARAMETERS

\section{7}

\section{STEAM FLDW = \\ STD DEV =}

1.174

1.212

3.25

0.01357

0.007

0.005

WATER FLDW $=0.0010$

0.000

0.00

0,0000

STI REV = 0.000

0.000

TOTAL FLÖW =

1.212

3.25

\section{FKL FROTOTYFE GPUOL TEST REGULTS}

\section{TEST NO. UNITS (KISIE)} WYLE

\section{FK}

$\begin{array}{rllll}\text { 172. STEAM FLOW } & =1.157 & 1.205 & 4.16 & 0.0481 \\ \text { STLIIEV } & =0.100 & 0.004 & & \\ \text { WATER FLIJW } & =0.000 & 0.000 & 0.00 & 0.0000 \\ \text { STLIEV } & =0.000 & 0.000 & & \\ \text { TOTAL FLOW } & =1.157 & 1.205 & 4.16 & \end{array}$

FKL PROTOTYFE SPQOL TEST RESULTS TEST NO. IINITS (KO/S) WYLE FK'L \% LIFF IIEVIATION

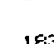
-10-

7.33

183
$-1.161$

$$
\text { sTD DEV }=0.002
$$

WATER FLAW $=0.000$

1.246

0.011

a. ocio

7.33

0.0875

0.00

0.0005

STI DEV $=, 0.0000 .000$

TOTAL FLOW $=-1.161-1.246$

7.33

\section{AVERAGE PKLL TEST PARAMETERS}

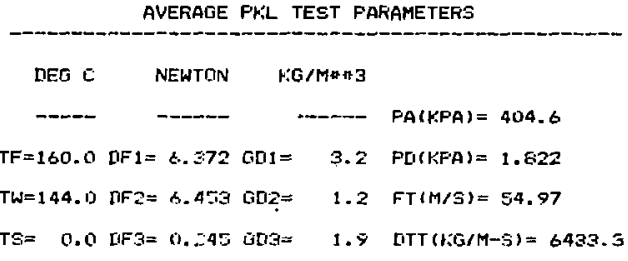

AVERAGE FKL TEST FARAMETERS

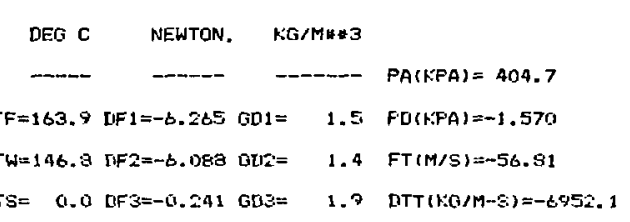


FKL FRITUTYPE SFUCIL TEST FEQLILTS

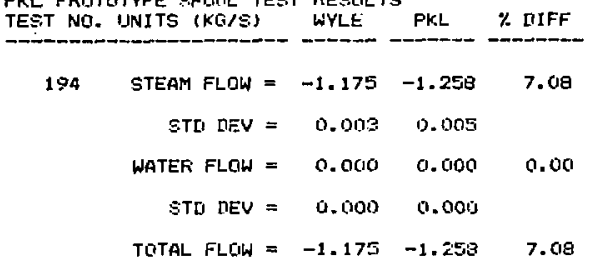

PKL PROTOTYPE SPOOL TEST RESLILTS

TEST ND. UNITS (KLT/S) WYLE

Pro

-

$$
252
$$

$$
\begin{array}{r}
\text { STEAM FLOW }= \\
\text { STD DEV }= \\
\text { WATER FLOW }= \\
\text { STD DEV }=
\end{array}
$$$$
0.60 \%
$$$$
0.616
$$

$$
0.000
$$$$
0.000
$$

TOTAL FLDH =

0.609

0.616

1.23
AVERAGE FR:L TEST FARAMEIEFS

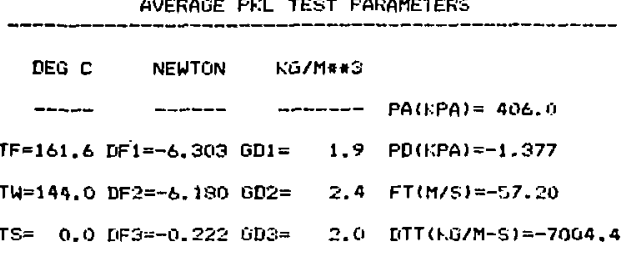

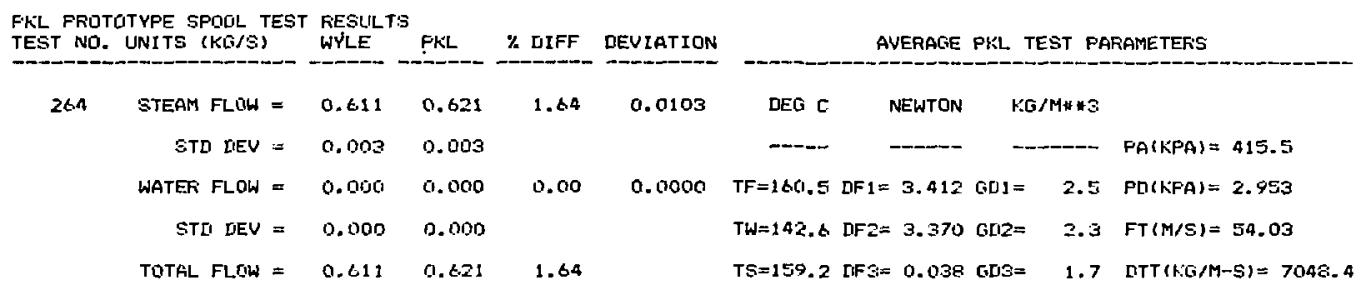


FKL PROTOTYPE SFOQL TEST RESULTS

TEST NO. LINITS (KG/S) WYLE

$$
\text { FKL }
$$

\% IIFF DEVIATION

203

$\begin{array}{rlrl}\text { STEAM FLOW } & =-0.613 & -0.701 & 14.35 \\ \text { STD DEL' }= & 0.003 & 0.001 & \\ \text { WATER FLOW } & =0.000 & 0.000 & 0.00 \\ \text { STE DEV } & =0.015 & 0.000 & \\ \text { TOTAL FLOW } & =-0.613 & -0.701 & 14.35\end{array}$

0.0897

2.0000

0.0000

$T F=160.5$ DF $1=-3.170 \mathrm{GD} l=$ $T W=-0.0$ DF2 $=-3.050 \quad 0 D 2=$

$T S=161.8$ DF $3=-0.026$ GD3 $=$
AVEFAGE PRIL TEST PAFAMETEFS

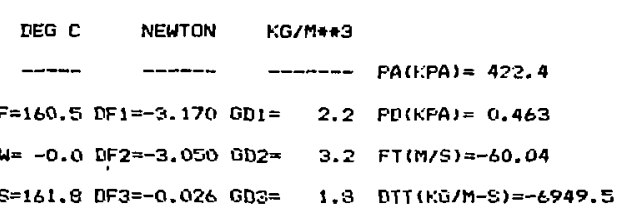

PKLL FROTOTYFE SPOOL TEST RESLLTS TEST NO. UNITS $\{$ H.LS AUEKALE FKLL TEST PARAMETERS

301)

$\begin{array}{rlrl}\text { STEAM FLOW } & =-0.6 .17 & -0.701 & 13.65 \\ \text { STO DEV } & =0.003 & 0.001 & \\ \text { WATEF FLOW } & =0.000 & 0.000 & 0.00 \\ \text { STI DEV } & =0.000 & 0.000 & \\ \text { TOTAL FLOW } & =-0.817 & -0.701 & 13.65\end{array}$

0.0355

DEG $C$

NEWTON

H:G/M**3

0.01000

-1- - - - -

- $P A(K P A)=422.6$

$T F=158.3 \quad D_{1}=-3.148 \quad 0[1=2.7 \quad P D(K F A)=0.457$

$\mathrm{TW}=0.0 \mathrm{DF} 2=-3.06: 3 \mathrm{GD} 2=$

4.9 FT(M/S) $=-60.02$

TS=159.8 DF $=-0.016 \cdot 505=$

1.5 DTT (KO/M-S) $=-6930.5$ 


\section{APPENDIX C}

PKL SPOOL PIECE TEST RESULTS FROM

TWO-PHASE WATER AND STEAM FLOW TESTS 
APPENDIX C

The data presented in this appendix give the test results for the two-phase water and steam flow tests and compare the results to the Wyle Laboratory test facility measured results.

The test numbers applicable to each of the three spools tested are given below:

$\begin{array}{cc}\text { Spool No. } & \text { Test No. } \\ 1 & 36-99 \\ 2 & 146-193 \\ 3 & 245-299\end{array}$

A test number is indicated for each test and the data is presented with the printouts in the following format:

- First row: Average steam flow data is given to the right of the heading "STEAM FLOW $=$ " with numerical values given in the "WYLE" AND "PKL" columns. In the same row, a percent difference calculated by equation (25) is given for these values under the col umn "\% DIFF", and a deviation calculated by equation (27) is given for steam flow rate under col umn "DEVIATION".

- Second row: Data is given on standard deviations associated with the Wyle and PKL steam flow rates, respectively, and is calculated using equation (24).

o Third and fourth rows: Similar data as for the above two rows is presented for water flows.

- Fifth row: Data is given on the total flow which is a sum of the steam and water flow rates already given. A percent difference between the total Wyle and PKL flow is given in the "\% DIFF" column and is calculated by equation (25). 
- To the right of the above data, under the general heading of "AVERAGE PKL IEST PARAMETERS", are given the average values for the engineering unit data obtained from each of the instrument channels. Identification for each of the abbreviations is given below:

TF = fluid temperature.

TW = wall temperature.

TS = superheated-steam temperature (applicable only to spool no. 4).

$\mathrm{DF} 1, \mathrm{DF} 2, \mathrm{DF} 3=$ drag screen transducer force for transducer nos. 1, 2, and 3.

$\mathrm{GD1}, \mathrm{GD2}, \mathrm{GD} 3$ = density along beam nos. 1, 2, and 3 of the densitometer.

$P A$ = fluid absolute pressure.

PD $\quad$ drag screen differential pressure.

FT = turbine velocity.

DTT = drag screen momentum flux. 


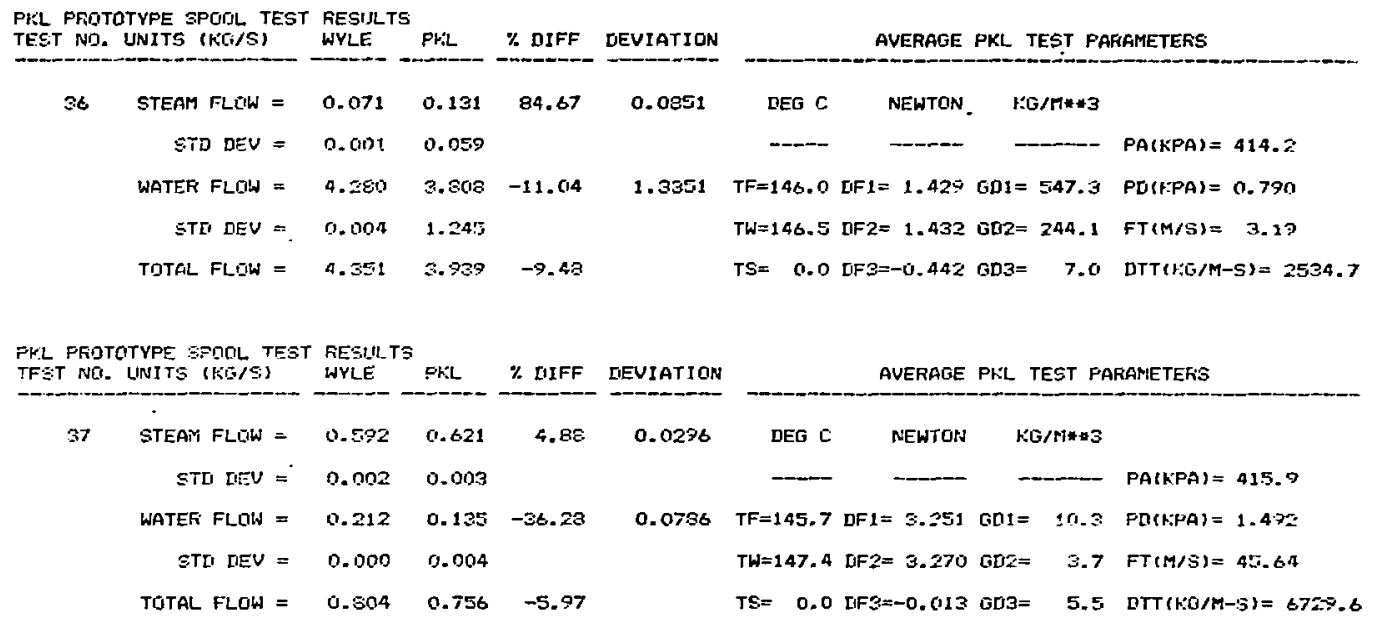

FíL FTUTOTYPE SPDOL TEST FESULTS

TEST NO. LNITS (KE/S) WYLE PKA

41
DEVIATION

0.0964

(1)

1.2842
AUERAGE PKL TEST PAFGMETEKS

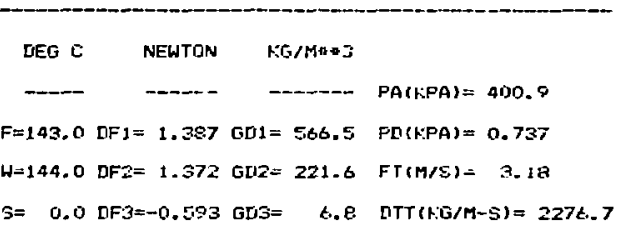


PFLL PROTOTYPE SPOOL TEST RESULTS TEST NO. UNYTS (KG/S) HYLE FKL 7 DIFF DEVIATION

42

\begin{tabular}{|c|c|c|c|c|}
\hline STEAM FLOW & $=$ & $0 . \pm 02$ & 0.627 & 5.97 \\
\hline STE LEV & $=$ & 0.002 & 0.003 & \\
\hline WATEFI FLOW & $=$ & $\mathrm{li.219}$ & 0.158 & -27.95 \\
\hline STD DEV & $=$ & 0.1903 & 0.004 & \\
\hline TUTAL FLC & $=$ & 0.811 & 0.785 & -3.17 \\
\hline
\end{tabular}

0.0383

NEG $C$

AVERAGE PKL TEST PARAMETERS

0.811

$0.785 \quad-3.17$
$0.0626 \quad T F=143.3 \mathrm{DF} 1=3.507$ GDI $=10.1 \quad \mathrm{PD}(\mathrm{H}, \mathrm{FA})=1.592$

$T W=145.3$ IF $2=3.556$ GL2 $=3.7$ FT $(M / S)=47.11$

$\mathrm{TS}=0.0$ [F $3=-0.076 \mathrm{GD}=5.0$ UTT $(\mathrm{KG} / \mathrm{M}-\mathrm{S})=7214.9$
PKL PROTOTYPE SPOOL TEST RESIMTS TEST NO. UNITS (N:G/S) WYLE

PKL. \% IIIFF DEVIATION 43
STEAM FLOW =
0.351
0.45730 .30
STI DEV =
0.002
0.004
WATER FLOW =
0.674
0.905
34.32
STD IIEV =
0.002
0.032
TOTAL FLLIWW =
1.025
1.363
32.95

PKL PROTGTYFE SFDUL TEST FESULTS
TEST NG. UNITS (HGIS)

44
PkLL \# DIFF DEVIATION

$0.0141-12.15$

0.0070

DES C

$K G / M=* 3$

STL DEV $=$

n. not

n.jon

WATER FLOW =

O. 1 EE

$0.003 \quad-39.74$

0.001

0.026

TÜTAL FLDH $=$

$0.135-33.32$
0.1089

AVERAGE PKL. TEST PARAMETERS

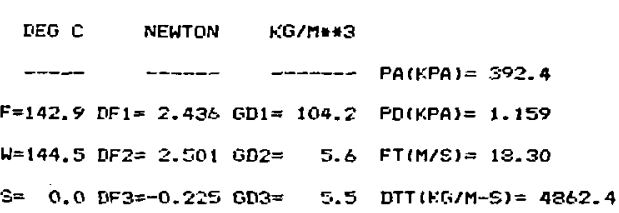

AVERAIJE FKL TEST PATAMETERS

-- - -

$0.0837 T F=143.7$ [IFI $=0.0 .35$ GDI $=233.5$ PUItiFA $=0.050$ $T H=145.0[1 F 2=0.005$ I.D? $=5.0 \quad F T(M / S)=1.44$

$T S=0.0[\mathrm{FF}=-0.010 \mathrm{Lin}=6.4$ [ITT $(1: 0, \mathrm{M}-\mathrm{S})=37.6$ 
FKL FFOTOTYFE SFOOL TEST RESULTS

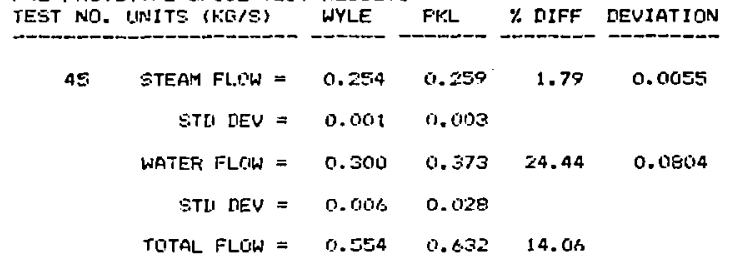

PKL PROTOTYFE SFODL TEST FEESULTS TEST NO. UNITS (KI:S S) WYLE TEST NO. UNITS (KG/S)

46

$\begin{array}{rlll}\text { STEAM FLLIW } & =0.714 & 0.750 & 5.10 \\ \text { STO LIEV } & =0.004 & 0.003 & \\ \text { WATEH FLIIW } & =0.218 & 0.16 .6 & -24.03 \\ \text { STL LIEV } & =0.001 & 0.005 & \\ \text { TOTAL FLUW } & =0.932 & 0.916 & -1.71\end{array}$

DEUIATION

\subsection{1}

(2)

0.0552 $T W=161.6$ DF2= 3.376 GD2= $T S=0.0$ IF $3=-0.049$ GDS=

AVERAGE PRLL TEST PARAMETEESE

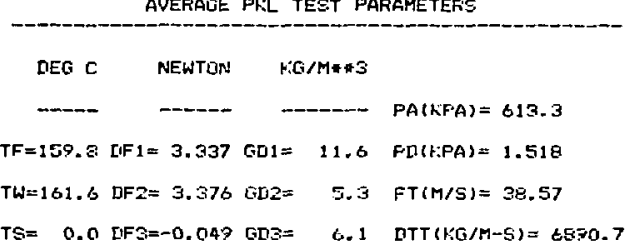

AVERALE FKL TEST PARAMETERS

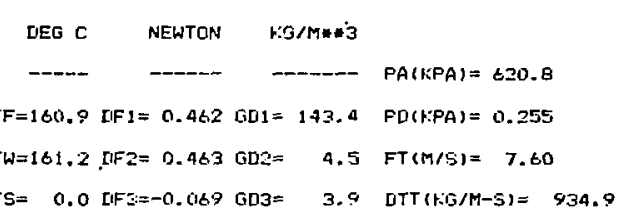

FKL PROTOTYPE SFOILL TEST RESLILTS TEST NO. UNITS (KGi/S) WYLE

\section{4}

$\begin{array}{rllll}\text { ETEAM FLOW } & =0.089 & 0.095 & 6.22 & 0.0057 \\ \text { ETU IEV } & =0.001 & 0.001 & & \\ \text { WATEF FLOW } & =0.255 & 0.399 & 36.34 & 0.1101 \\ \text { STD DEV } & =0.000 & 0.019 & & \\ \text { TOTAL FLOW } & =0.302 & 0.494 & 29.32 & \end{array}$

FKL \% DIFF DFUIATION

AVERAGE FHL TEST PARAMETERS

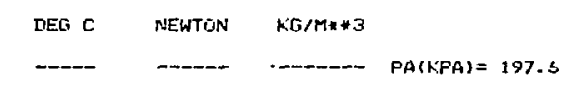

$T F=120.3 \quad\left[F F_{1}=0.187 \quad[\quad[11=206.2 \quad P O(1 F F A)=0.102\right.$

$T W=121.4[I F 2=0.1 \% 4 \mathrm{G}[2=4.0 \mathrm{FT}(\mathrm{M} / \mathrm{S})=4.23$

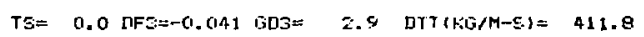


FKL FROTOTYPE SPOOL TEST RESLLTS TEST NO. HNTTS (HITS) WYLE FH:L 55

$\begin{aligned} \text { STEAM FLOW } & = \\ \text { STI IEV } & = \\ \text { WATER FLOW } & = \\ \text { STU IEV } & = \\ \text { TOTAL FLIIW } & =\end{aligned}$ $0.3 \leqslant 0$ $0.444 \geq 3.41$

0.0859

0.005

0.001

0.295

$0.239-18.89$

0.0769

0.001

o. 1093

0.684

4.36

$T S=0.0 \mathrm{pF} 3=-0.043 \mathrm{GD} 3=$

\section{AVERAGE PKL TEST PARAMETERS}

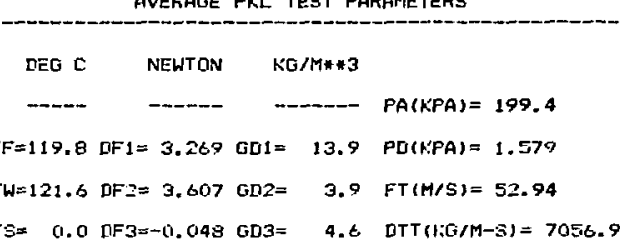

PKL FROTOTYFE SPGQL TEST FESHLTS

\begin{abstract}
TEST NO. UNITS (k:Ci/S)
\end{abstract}
W'YLE

FKL

\% DIFF

IEEUIATION

56

STEAM FLETW =

0. 05

$0.126 \quad 125.62$

0.0728

ETU LEV $=0.001$

0.012

WATER FLOW $=3.100$

$2.525-13.56$

STII DFU $=0.006$

0.851

TOTAL FLOU! =

‥ 156

$2.651-15.99$

PLL PROTOTYFE EPUILL TEET REGIILTS

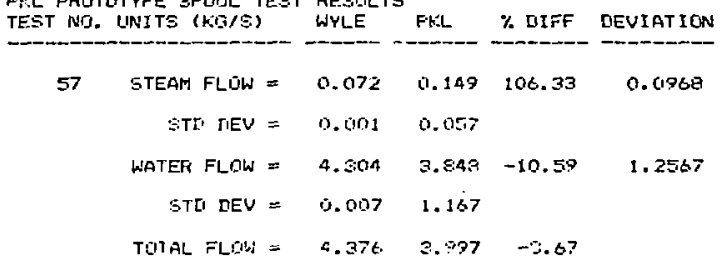

AVERAGE PKL TEST PAKAMETERS

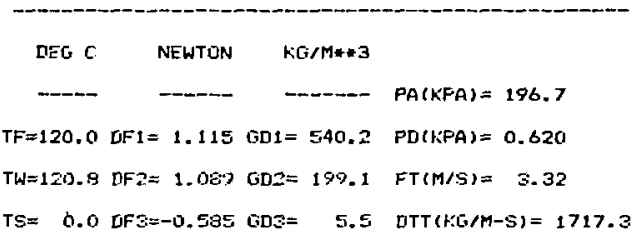

AVERAGE PHLL TEST FARAMETEFS

$\begin{array}{lll}\text { DEG C } & \text { NEWTON } & \text { KG/MGH3 } \\ & & \\ - & - & -\end{array}$

$T F=145.1[F \mid=1.531$ GDI $=591.8 \quad P D(F, P A)=0.869$ $\mathrm{TW}=145.1 \mathrm{DF} Z=1.525 \mathrm{GD} Z=243.1 \quad \mathrm{FT}(\mathrm{M} / \mathrm{S})=3.20$ $T S=0.0[\mathrm{~F} \xi=-0.619 \mathrm{GD} Z=6 . ? \mathrm{DTT}(1 . \mathrm{G} / \mathrm{M}-\mathrm{S})=2552.7$ 
FK'L PROTOTYPE SPLOL TEST FESLILTS

TEST NO. LNITS (KETSS)

$$
\text { WYLE }
$$

PKL

\% DIFF DEVIATION

58

$\begin{array}{rlrr}\text { STEAM FLOW } & =0.579 & 0.615 & 0.27 \\ \text { STO DEV } & =0.001 & 0.002 & \\ \text { WATEF FLOW } & =0.215 & 0.154 & -28.74 \\ \text { STE DEV } & =0.001 & 0.004 & \\ \text { TOTAL FLOW } & =0.795 & 0.789 & -3.24\end{array}$

0.0371

0.0505

0.0835

\section{$T W=144.6 \quad \mathrm{DF} 2=3.454$ GD $2=$}

$\mathrm{TS}=$

0.0 IfF3 $=-0.048$ GDI3=
AUERAGE PKL TEST PARAMETERS

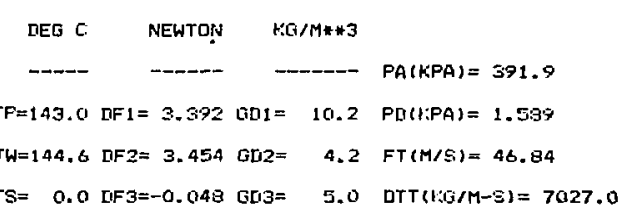

PKL PROTOTYPE SPÜOL TEST RESULTS

TEET NO. LINITS (HIS/S) WYLE PKL \% DIFF DEVIATION

TEET NO. LNITS (TITIS) WYLE PRL \% DIFF DEUIATIAN

59 STEAM FLOW =

$\begin{array}{llll}0.155 & 0.265 \quad 71.26 & 0.1129\end{array}$

DEG C NEWTON H:G/M*3

STD DEV $=0.0010 .006$

(1)

-

WATER FLOW $=2.565 \quad 2.626 \quad-3.36 \quad 0.5506$

$\mathrm{TF}=160.1 \mathrm{nF} 1=1.327 \mathrm{GD} 1=4.91 .4 \quad \mathrm{PM}(\mathrm{FPA})=0.771$

STD IEV $=0.000 \quad 0.493$

$\mathrm{TW}=159.7 \mathrm{DF} Z=1.31 \mathrm{GN2}=17.3 .1 \quad \mathrm{FT}(\mathrm{M} / \mathrm{S})=3.5_{2}$

TOTAL FLOW $=\quad 3.020 \quad 2.891 \quad-4.27$

$\mathrm{TS}=0.0 \mathrm{IF}=-0.549 \mathrm{GD}=8.5$ DTT $(60 / \mathrm{M}-\mathrm{S})=2205.2$

FKL FROTQTYFE SFODL TEST FESI-LTS

TEST NO. UNITS (KIG/S) WYLE PKL

60
STEAM FLOW =
0.065
0.03
26.01
STII IIEV =
0.003
0.0105
WATER FLOW =
0.429
0.536
25.13
STD DEV $=0.001$
0.060
TGTAL FLOW =
0.453
$0.17 \quad 25.25$

EEVIATION

\section{$0: 0177$}

REG C NEWTON

--n

--con

$f: G / M+43$

0.1251

$\mathrm{TF}=180.6 \quad \mathrm{DF} 1=0.065 \mathrm{GD} 1=4$ $T W=160.8[I F 2=0.064 \quad G I 2=$
$\mathrm{TS}=0.0 \mathrm{DF} 3=-0.005 \mathrm{GD}=$

AVERAGE FKL TEST PARAMETERS

\section{- FA(1:FA) $=617.3$}

16.2 FIUKFFA $=0.046$

64.7FT(M/S) $=1.2 \mathrm{G}$

4.7 ПТT (1:G/M-S) $=154.1$ 
FYL PRIIIOTYPE SPCIOL TEST RESILTS TEST NO. LNITS (KIVS) WYLE

PKL \% DIFF tueviation

6

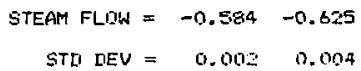

WATEF FLOW $=-0.168-0.17 t$

4.51

GTD DEV $=0.001$

0.005

TOTAL FLLIW $=-0.752-0.500$

6.43

FKL PROTOTYFE SFOOL TEST RESLILTS TEST NO. INNITS (HEIS) WYLE F+k

70
0.0418

DEG C

$=$

0.0091

$T F=144.8$ OF $1=-3.265$ G $\mathrm{DI}_{1}=$ $\mathrm{TW}=145.7 \mathrm{nF}:=-3.28 \mathrm{GN2}=$ $T S=0.0$ [IF $3=0.065$ GDS=

\section{AVERALE PKL TESTT FARAMETERS}

NEWTON. KG/M**3

$F A(K F A)=406.4$

5.0 PD(YFA) $=-1.343$

5. $0 F T(M / S)=-45.69$

\begin{tabular}{|c|c|c|c|c|c|}
\hline STEAM FLOW & $=$ & -0.350 & -0.200 & -42.76 & 0.1527 \\
\hline STD IUEV & $=$ & 0.003 & 0.003 & & \\
\hline WATER FLOW & $=$ & -0.654 & -1.389 & 100.17 & $0.70 \% 9$ \\
\hline STD DEW & $=$ & 1). 002 & 0.024 & & \\
\hline TOTAL FLDW & $=$ & -1.044 & -1.589 & 52.25 & \\
\hline
\end{tabular}

$7.0[\mathrm{ITT}(1: \mathrm{G} / \mathrm{M}-\mathrm{S})=-7132.0$

\begin{tabular}{|c|c|c|c|c|}
\hline DEG $c$ & NEWTEIN & & $F / M+3$ & \\
\hline$--\infty$ & $\ldots$ & & $\cdots$ & $P A\{K P A\}=409.0$ \\
\hline 45.2 & $\mathrm{DF} 1=-2.497$ & $\mathrm{GO} 1=$ & 21.5 & $P Q(k F A)=-1.573$ \\
\hline$W=145.9$ & $\mathrm{DF} Z=-2.390$ & and- & 10.4 & $F T(M / S)=-15.97$ \\
\hline
\end{tabular}

AVERAGE FKIL TEST PARAMETERS

FKL FFOTOTYFE EFGUL TEST REEULTE

TEST NUL. HNITE (KIT/S) WYLE

FKL \% DIFF EIEVIATILN AVERAGE FHL TEST FARAMETEKS

\begin{tabular}{|c|c|c|c|c|c|}
\hline STEAM FLGN & $=$ & -0.044 & $-0.02 s$ & -40.74 & 0.0186 \\
\hline STI I!EV & $=$ & 0.000 & 0.004 & & \\
\hline WATEF FI_CW & $=$ & -0.142 & -0.129 & -9.80 & 0.0289 \\
\hline STE IIEV & $=$ & 0.001 & 0.025 & & \\
\hline TOTAL FLSW & $=$ & $-0.13 s$ & -0.15 .4 & -17.12 & \\
\hline
\end{tabular}

74

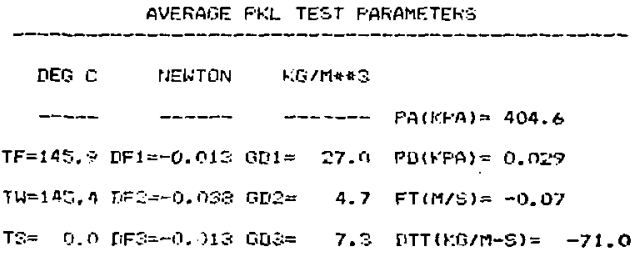


FKL FTIOTOTYPF SFÖIL TEST RESILTS

TEST NI. INITS (KI/S) WYLE

FH:L

\% RIFF IEVIATION

75

$$
\begin{array}{rlrl}
\text { STEAM FLOW } & =-0.073 & -0.073 & -0.65 \\
\text { STL DEV } & =0.002 & 0.053 & \\
\text { WATER FLOW }= & -4.320 & -4.703 & 10.25 \\
\text { STE DEV }= & 0.002 & 0.839 & \\
\text { TOTAL FLCIW }= & -4.303 & -4.835 & 10.07
\end{array}
$$

PKL FROTGTYPE SPRIIL TEST REAILTS

TEST NO. IIN1TS (KG;S) WYLE

73

$$
\begin{array}{rlr}
\text { STERM FLFW }= & -0.060 & -0.0187 \\
\text { STO LIEV }= & 0.001 & 0.002 \\
\text { WATER FLOW }= & -0.044 & -0.617 \\
\text { STD TIEV }= & 0.001 & 0.035
\end{array}
$$$$
44.51
$$

$33_{n} .5$

TOTAL FLDW $=-0.504-0.70 \%$

39.53

PKL FFOTOTYFE SFIOL TEST RESULTS

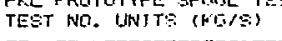

FESUL

\begin{abstract}
Pre
\end{abstract}

\begin{tabular}{|c|c|c|c|}
\hline NEG C & NEWTON & $k+\Delta / t+3$ & \\
\hline -- & $----\infty$ & ----- & $F A(+. F A)=625.7$ \\
\hline$F F=161.2$ & {$[\mathrm{IF} 1 \doteq-1.6 .39$} & $6[11=300.5$ & $P[1(1: F A)=-0.790$ \\
\hline$T W=1 \leqslant 1,4$ & [IF $2=-1.5 .44$ & $\operatorname{crr}_{2}=2=2.6$ & $F T(M / S)=-4 . n$ \\
\hline
\end{tabular}

FKL

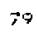

40.150 .0694

24.6 .3

0.7773
0.0273

0.1795 TH=161.7 [IF $Z=-0.097 \quad G[2=121.7$ FT $(M / 5)=-1.37$ $T E=0.0[F G=0.017 .013=$
DES C

AVERALE FH:L TEST FARAMETERS.

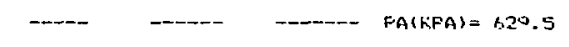

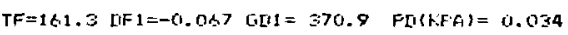

$4.1[\mathrm{TT}(1, \mathrm{i} / \mathrm{M}-\mathrm{s})=-1 E 7.7$

AVERAGE PKL TEST PARAMETEFS

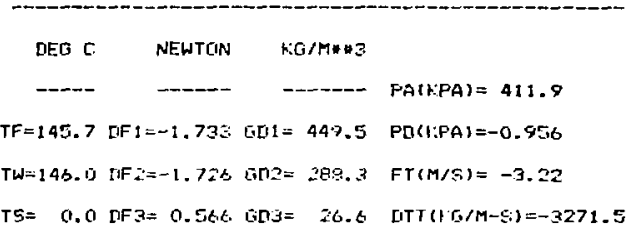

AUERAGE FHLL TEST PARAMETER:. 
FHL PRLTOTYFE SFOCL TEST AESILT:

TEST NÜ. LINIYS (HIOLSS)

$$
\text { WYLE }
$$

PR:L

$\%$ IIIFF

DEVIATION

80

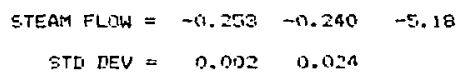

69.95

WATER FLOW $=-0.290-0.49$

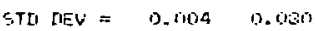

TGTAL FLOW $=-0.54 \% \quad-0.731$

34.58

\section{FKL FRITITTYFE EFGUL TEET RESILTS}

TEST NÜ. UNITS (HLN) WYLE

FHIL \% IIIFF [IEVIATION

e1

$$
\begin{array}{rlrl}
\text { STEAM FLOW } & =-0.713 & -0.737 & 3.70 \\
\text { STI DEV } & =0.00 .5 & 0.007 & \\
\text { HATER FLOW } & =-0.180 & -0.192 & 20.09 \\
\text { STI IEV } & =0.001 & 0.029 & \\
\text { TOTAL FLOW } & =-0.278 & -0.930 & 5.37
\end{array}
$$

FKL PROTUTYFE SFOML TEST RE:LLLTE

TEST NO. INNITS (b,fi/S)

WYLE

PKLL

\% DIFF DEVIATION

82
32. UE

a.me 2

$-0.239$

$19.7 \%$

STI DEN =

$0,1=1$

TOTAL FLOH = -r. EST -0.EI:

\begin{tabular}{|c|c|c|c|c|}
\hline IEE C & NEHTON & & $/ M+3$ & \\
\hline$-\infty$ & $-\infty-\infty$ & - & -- & $F A(K . F A)=6.22 .4$ \\
\hline$=160.3$ & $\mathrm{BF1}=-0.513$ & $\mathrm{~B}[\mathrm{OI}=$ & 50.8. & $P[1(t, F \cdot A]=-0.2 E B$ \\
\hline$=18.1 .4$ & IIF $2=-0, .22$ & $602=$ & 10.3 & $\mathrm{FT}(\mathrm{M} / \mathrm{s})=-8.5 \mathrm{\nu}$ \\
\hline
\end{tabular}

22.60
0.0274

AVEKAGE FF:L TEST PARAMETFFE

0.0208

0.0495

$T F$

$T W=160.7 \mathrm{DF} 2=-3.124 \quad 602=$

AVERAGE FKLL TEST FARAMLTEHE

DEG C NEWTUN HLIM\#\#

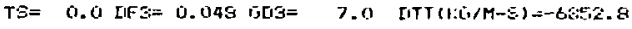

AVERALE FHIL TEST PARAMETEFS:
0.1212
IES E NEWTUN
$1: 0 / m+43$
- - - - -
$0.0200 \quad T F=120.6$ LF $1=-4$. 3a1 ntil $=$
5. $3 F[1(t . F A)=-1.6060$
$\mathrm{SN}=121.6$ IIF $2=-4.010$ GN2=
6.0 FT $(H, N)=-5.0 \%$

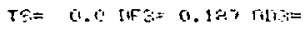
6. 
PKL FRUTATYFE SFERIL. TEST RE AH.TS IEST NO. UNITS (HG/S)

$$
\text { WYLE }
$$

Pk:L

83
\% DIFF LEVIATISN o. 0332

DES $\mathrm{L}$
AUKFAGE FHL TFET FATEATLTFE

\begin{tabular}{|c|c|c|c|c|c|}
\hline \multirow[t]{2}{*}{1.17} & 0.01332 & DEG $\mathrm{L}$ & NEWTUN & $1.6 / M+123$ & \\
\hline & & $-\cdots$ & $-\cdots--$ & $m-\infty-\infty$ & Find Frit $=190.0$ \\
\hline \multirow[t]{2}{*}{78.59} & 0.3513 & $T F=119.9$ & {$[\mathrm{IF})=-0.3 \mathrm{~s} \theta$} & $B_{1}[I]=11, \therefore 1$ & Frid F.A $=-6.104$ \\
\hline & & $T W=t 21,3$ & I.F $E=-1), 20$ & $6 \mathrm{r}_{1}=1 \therefore 7$ & $F T(M, \pi)=-T .44$ \\
\hline & & $T S=0.0$ & {$\left[H F S=0, n_{S}=\right.$} & {$[\cdot[1:=$} & {$[T](M, M-3)=-890.5$} \\
\hline
\end{tabular}

FK'L PROTOTYFE SFOOL TEST RESULTS TEST NO. LINITS (KG/S) WYLE FkiL \% IIIFF LEVIATICIN

$$
84
$$

$$
\begin{array}{rlrl}
\text { STEAM FLÜ } & =-0.054 & -0.131 & 141.86 \\
\text { STL LIEV } & =0.001 & 0.063 & \\
\text { WATER FLOW } & =-3.032 & -2.306 & -7.45 \\
\text { STO DEV } & =0.0016 & 1.164 & \\
\text { TOTAL FLOW } & =-3.036 & -2.537 & -4.83
\end{array}
$$

\subsection{7}

GIELT 0

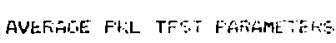

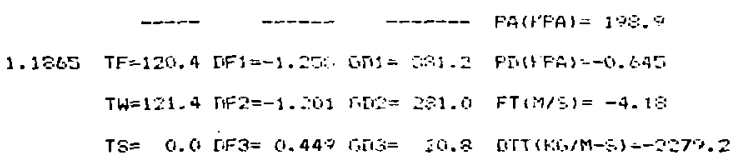

PKL PROTOTYFE SPUIL TEST FESILTS

TEST NO. UNITS (KIO/S)

WYLE

Pr:L

\% LIFF DEVIATION

AUERAGE FHL TEET FARAMETERS

95
0.0357 TIEE E NEWTINN 1:0/MH or 3

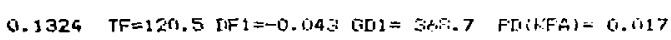

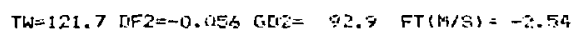

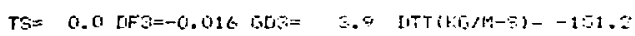


FKL PROTOTYFE SFCIOL TEST RESULT:S TEST NO. LINITS (KIS/S) WYLF PKI

$\$ 1$
$8.16 \quad 0.0434$

ETI IEV $=$ D. 0.1050 .004

WATEF: FLOHN $=-0.213$

STD DEV $=$

0.000

0.007

TOTAL FLIIW

$-0.700-0.802$

10.33

\section{AUERAGE PFLL TESTT PARAMETEKS}

LIEG C NEWTLIN. KG/M**3

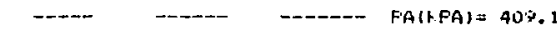

$T F=145.0 \mathrm{NF} 1=-3.533 \mathrm{GD} 1=5.0 \mathrm{~F}[\mathrm{~W}(1) \mathrm{PA})=-1.3 \mathrm{a5}$

$T W=140.1$ [IF $2=-3.422$ Gn $2=5.6 \quad F T(M / S)=-4.3 .17$

$T S=0.0$ IFS $=0.209 \mathrm{GD}=$

7.4 DTT $(K .0 / M-S)=-742 Z .4$
FH:L FRITGTYFE SFOUL TEST RESIJLTS WYLE Fit. \% DIFF

DEVIATION

22

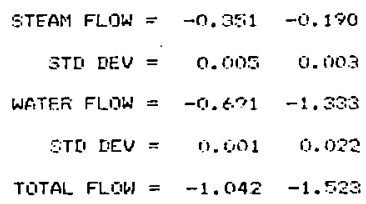

TEST NO. LNITS (T E/S)

\section{AVERAGE FHLL TEST FAKAMFTERS}

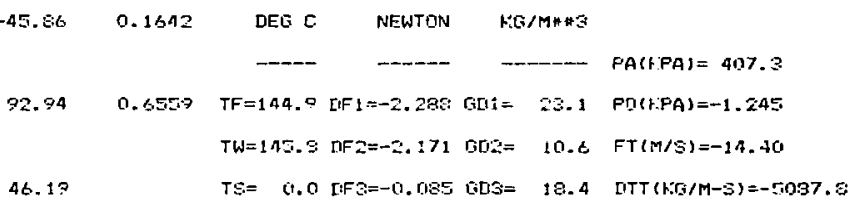

FKL PROTOTYPE SFODL TEST FEESILTS TEST MO. UNITS (YTOSS) WYLE PHI Pr.I. 2 IITFF DEUTATION AVEFAGE PKL TEST PARAMETERS

93

$$
\begin{aligned}
& \text { ETEAM FLOH }=-0.597 \quad-0.609 \\
& 6.59 \\
& \text { STI DEV = 0. } 201 \quad 0.0124 \\
& 17.59
\end{aligned}
$$$$
0.0399
$$$$
\text { IIEG } C \text { : }
$$$$
\text { NEWTON }
$$$$
K G / M * * 3
$$

0.0955

$T F=144.9[\mathrm{~F} /=-3.395, \mathrm{G}[1]=$

5. 4 PII(K:PA) $=-1.324$ $T W=145.0$ [PF $2=-3.323$ G[1]=

5. 0 FT(M/S) $=-44.71$

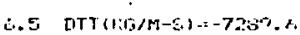


PHL FTIOTOTYFE SFODL TEST RIESULTS TFET NG. UNITS (HE)S) WYLE FI:L 94

$$
\begin{array}{rlrl}
\text { STEAM FLOW } & =-0.070 & -0.089 & 20.11 \\
\text { STL IIEU } & =0.001 & 0.014 & \\
\text { WATER FLOW } & =-4.270 & -4.441 & 4.47 \\
\text { STI IEV } & =0.013 & 0.101 & \\
\text { TOTAL FLOW } & =-4.340 & -4.548 & 4.80
\end{array}
$$

FRL FFOTOTYFT :FFONI TFET PESIILTS

TESTT NL, LNITS (K.L/E) WYLE FYLL $\%$ DIFF DEVIATION wo

\begin{tabular}{|c|c|c|c|c|c|c|c|c|c|c|}
\hline $\begin{array}{l}\text { FEL FnOT } \\
T=51 \text { NIO. }\end{array}$ & $\begin{array}{l}\text { TYPE FFOTL T } \\
\text { UNITS (NG/S) }\end{array}$ & TEST & $\begin{array}{l}\text { FEOLULT } \\
\text { WYLE }\end{array}$ & 5 Ft.L. & $\%$ IIFF & EIEVIAT ION & \multicolumn{4}{|c|}{ AUEFAIEE FIIL TEST PARAMETERS } \\
\hline \multirow[t]{5}{*}{ so } & STEANI FLOH & $=$ & -10.070 & -0.125 & 79.90 & 0.0623 & DEÓ C & NEWTEN & $Y: G / M=+3$ & \\
\hline & STI DEV & $=$ & 0.00 & 0.019 & & & --- & ---- & $--\infty---$ & $P A(K P A)=\$ 9 \%$ \\
\hline & WATHF FLQH & $=$ & -4.365 & -4.017 & -6.02 & 0.4474 & $T F=14 A .3$ & {$[\mathrm{~F} \mathrm{i}=-1 ., 4 \leqslant 0$} & $G[11=445.1$ & $P n(t P A)=-0.80 A$ \\
\hline & STO NIEV & $=$ & 0.006 & 0.230 & & & $T W=143.3$ & $\mathrm{DF}=-1.417$ & $\operatorname{SLE}=27 \% .4$ & $F T(m / S)=-3.14$ \\
\hline & TOTGI_ FLOW & $=$ & -4.430 & -4.143 & -6.65 & & $T S=0.0$ & $\mathrm{r} F 3=0.6 .43$ & $0.03=25.7$ & $\operatorname{tiT}(10,1 M-5)=-2533.6$ \\
\hline
\end{tabular}

$$
\begin{aligned}
& \text { STEAM FLLIW }=-0.589 \quad-0.631 \\
& \text { STt [स: }= \\
& 0.002
\end{aligned}
$$$$
7.20
$$

8.97
AVERAGE FILL TEET FARAMETEFS

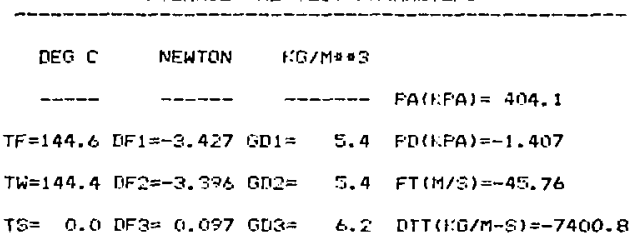




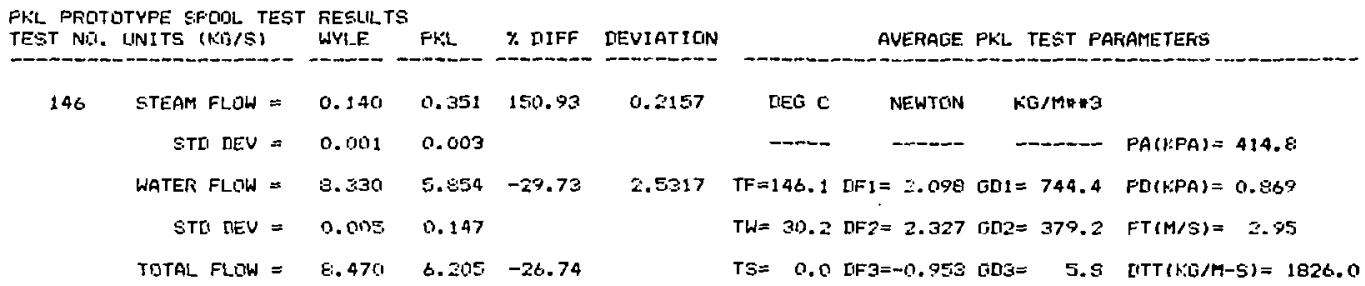

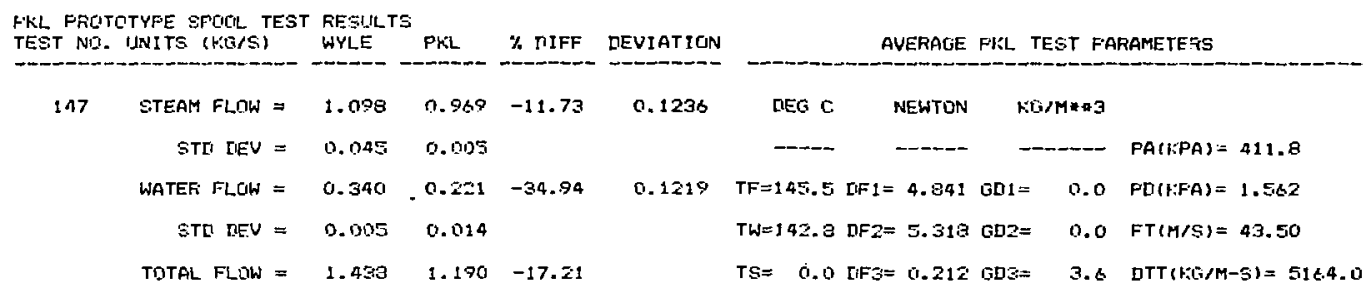

PKL PROTOTYFE SPOOL TEST RESLLTS
TEST NO. UNITS (KOIS:
WYLE


FHL FHOTGTYFE SFIM. IEST RFEILTS

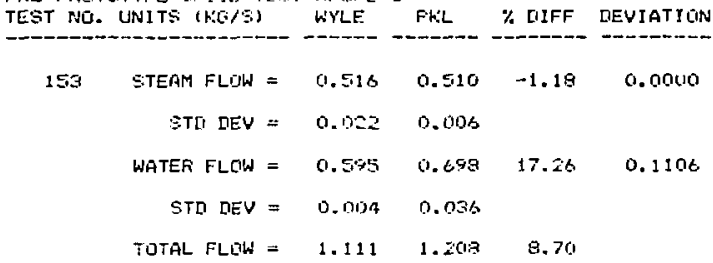

FVIL PROTOTYFE SFOCL TEST RESLILTS

TEST NÖ. UNITS (1:D/S) WYLE

$F$

\% IIIFF DEVIATICN

154

\subsection{2}

$0.00 \%$

0. $0131-75.27$

1. .007

TOTAL FLO'N
$1.439-19.45$
AVERALE PH:L TESTT FAFAMETERS

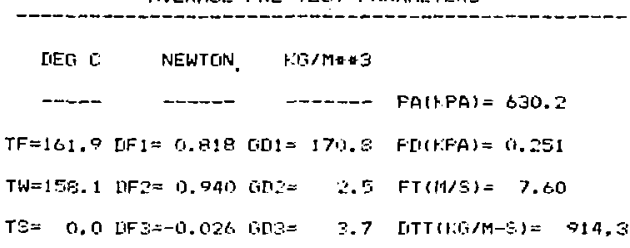

AVERACE FKL TEST FARAMETERS

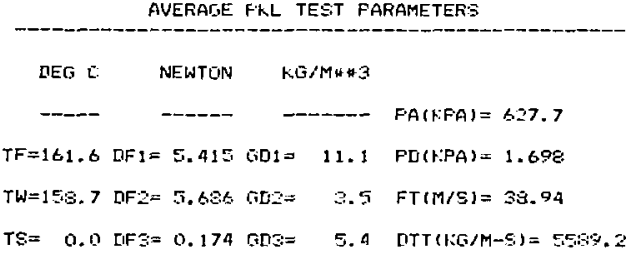

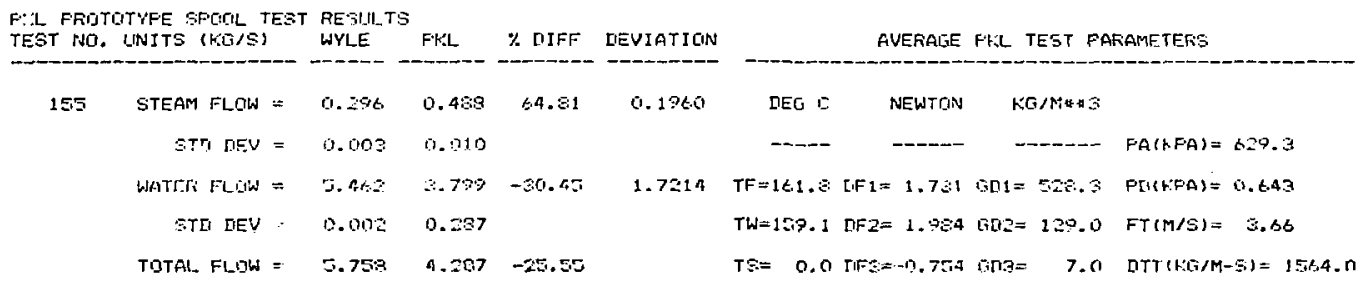


FKL PROTOTYFE SPLOL TEST RESLLTS

TEST NLI. IINITS (KOIS) WYLE PHIL 156 STEAM FLOW =

0.120

$0.093-22.68$

0.0268

STI DEV =

0.003

0.003

WATER FLOW $=$

0.834

$0.479-42.57$

ETLI DEV

o. ine.

0.097

TOTAL FLÖH $=0.55,4$

$0.572-40.06$

PKL FROTOTYPE SFCULL TEST RESILTS

TEST NO. UNITS (KG/S) WYLE

PKL. \% $\mathrm{EIFF}$

DEVIATION

15

\begin{tabular}{|c|c|c|c|}
\hline STEAM FLÏH = & $0.0 \neq 0$ & $0.7 \% 1$ & 13.23 \\
\hline STD IIEV = & 0.1223 & 0.009 & \\
\hline WATEF FLOW = & 1.350. & t. 118 & -17.19 \\
\hline STD DEV = & 0.001 & 0.0513 & \\
\hline TOTAL FLOW & $2.04 \%$ & 1.900 & -6.81 \\
\hline
\end{tabular}

DEG C

K'G/M**3

---

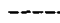

$0: 2433$

$\mathrm{TF}=146.1 \quad \mathrm{DFI}=3.695 \mathrm{GDI}$

S0.0 FD(N:PA $)=0.970$

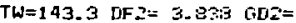

$T S=$

0. 0 DF $3=-0.891$ 6D3=

$4.7 F T(M / E)=17.77$

3.3 NTT $(1, G / M-5)=3382.7$

FKL PRGTOTYFE SFONL TEST RESIILTS

TEST NID. UNIT: (FIG/S) WYLE FKL \% IITFF DEVIATION

150 STEAM FLOW $=1.004 \quad 1.066 \quad-2.59 \quad 0.0000$

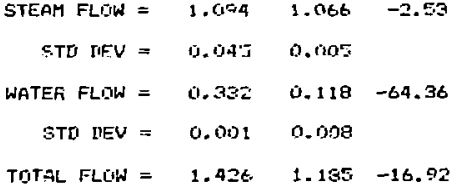

DEG C

TOTAL FLON $=1.426$. $1.195-16.92$

0.2132 TF=144.6 DFI= 5.025 ODI = $T W=142.3 \quad \mathrm{QF}:=3.412602=$ $T S=0.0$ DF3 $=0.132$ Gn3 $=$
$K G / M a=3$

$P A(K P A)=4(1: 2.2$

10.4 Frit:PA) $=1.61 \mathrm{t}$

$2.7 \mathrm{FT}(\mathrm{M} / \mathrm{S})=44.52$

4.4 DTt $(4: 0 / M-E)=5259.0$ 
FWL PROTOTYPE SPOOL TEST RESILTS TEST NO. UNITS (K'G/S) WVLE PKL \% LIFF DEUIATION

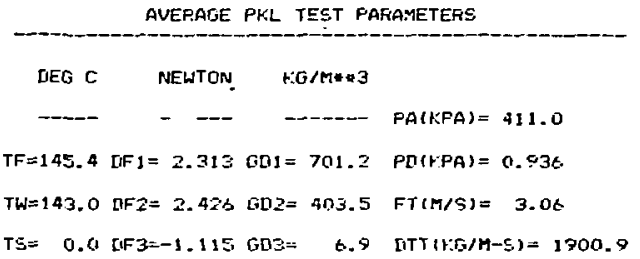

PKL PROTOTYFE SPCOL TEST RESILTS TEST NO. UNITS (K,O/S) WYLE PF:

\begin{tabular}{|c|c|c|c|c|c|}
\hline \multirow[t]{5}{*}{162} & STEAM FLOW = & 0.703 & 0.779 & 10.77 & 0.0775 \\
\hline & STD NEV =ं & 0.001 & 0.006 & & \\
\hline & WATER FLחW = & $0.5 \% 5$ & 0.332 & -44.27 & 0.2693 \\
\hline & STD [IEV = & 0.002 & 0.015 & & \\
\hline & TOTAL FLOW = & 1.298 & 1.110 & -14.46 & \\
\hline
\end{tabular}

PKL PROTOTYPE SPOCL TEST RESULTS TEST NO. UT.ITS (1:0/O) WYLE PKL * DIFF LEVIATION

$\begin{array}{rlll}163 \quad \text { STEAM FI_OW } & =0.131 & 0.184 & -9.57 \\ \text { STL LIEV } & =0.001 & 0.0012 & \\ \text { WATER FLOW } & =0.590 & 0.551 & -0.2 .3 \\ \text { STD DEV } & =0.0101 & 0.0: & \\ \text { TOTAL FLOW } & =0.771 & 0.715 & -7.32\end{array}$
0.2301
2.4458

AVERAGE PKIL TEST FAKAMETEfiS

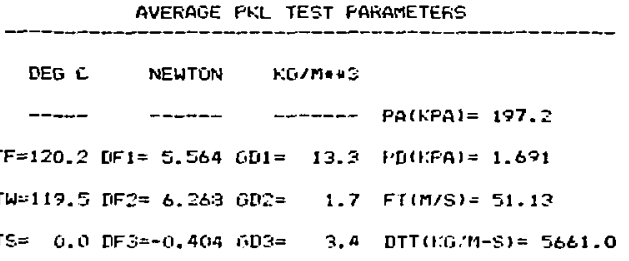

AVERAGE PNL TEST FAFAMETER:
-

0.0177

\section{DEG C NEWTON KG/M**Z}

- - -

$T F=120.2 \quad[F 1=0.246 \quad[D 1=2 \% \% \quad F(1(1 . F A)=0.072$ $T H=113.6 \mathrm{DF} .2=0.334$ G[OZ= 0.8 FT $(M / 3)=4.21$

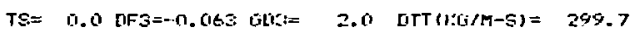


FKL FIYTOTOTYE SFQULL TEST RESLLTS

\begin{tabular}{|c|c|c|c|c|c|}
\hline ST ND & UNITS (KG/S) & WYLE & FKL & $\%$ LIIFF & DEVIATION \\
\hline \multirow[t]{5}{*}{164} & STEAM FLOH = & 0.106 & 0.230 & 117.34 & 0.1271 \\
\hline & STI nEV = & 0.001 & 0.008 & & \\
\hline & WATEF FLOW = & 5.923 & 4.362 & -25.09 & 1.5523 \\
\hline & STD NEV = & 0.0104 & 0.431 & & \\
\hline & TOTAL FLOW $=$ & 5.729 & & -22.55 & \\
\hline
\end{tabular}

PKL PROTOTYFE GFTIOL TEST FESTLTS

TEST PROTOTYFE GPFOL TEST KESII

TEST NO. LNITS (KG/S) WYLE

FKL

\% LIIFF LIEVIATION

170

\begin{tabular}{|c|c|c|c|}
\hline STEAM FLUW = & 0.141 & 0.352 & 140.35 \\
\hline STD DEV $=$ & 0.001 & 0.003 & \\
\hline WATER FL IW = & 8.273 & 5.515 & -23.51 \\
\hline STD REV = & $0.10 \mathrm{~s}$ & 0.140 & \\
\hline TÜTAL FL & 5.414 & 6.267 & -25.52 \\
\hline
\end{tabular}

0.2149

(1)

DEG C NENTIN HEIMMES

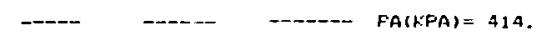

$2.4108 \quad T F=14 t .1 \quad[(F 1=2.183 \quad 00 t=734.3 \quad$ P(IFFA $)=0.85$

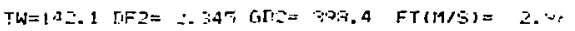

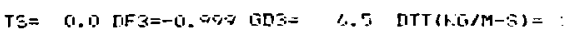

PAL PROTOTYFE BFGLLL TEST RESULTE TEST NO. UNITS (KEIE) WYLE FKL \% DIFF DEVIATIQN AVEKALE PFiL TEST faRAMETEFS

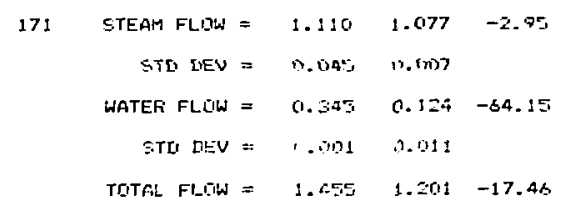

[IEG : NEWTON KISTMar

--.- - - - - -

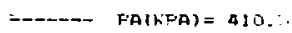

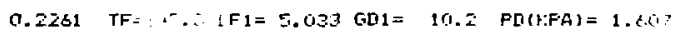
TW-::7.7 $2=5.37 \%$ Griz= 2.5 FT(M/S)= $44 . \mathrm{rl}$

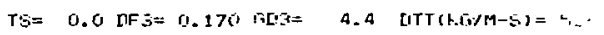


FKL PROTOTYPE SPGIOL TEST RESLLTS TEST NO. UNITS (KGIS) W WYE

PHL \% DIFF IEVIATIUN AVERAISE FKIL TEST FARAIHFTER

175

$$
\begin{array}{rlrr}
\text { STEAM FLOW } & =-1.102 & -1.129 & 2.40 \\
\text { STD DEV } & =0.045 & 0.004 & \\
\text { WATEF FLOW } & =-0.341 & -0.231 & -32.13 \\
\text { STO DEV } & =0.001 & 0.007 & \\
\text { TOTAL FLCIW } & =-1.443 & -1.360 & -5.76
\end{array}
$$

PKL PROTOTYPE SPOTLL TEST RESIILTS

TEST NO. UNITS (KTIS) WYLE

FKL

\% IIIFF

176

$$
\begin{array}{rlrl}
\text { STEAM FLOW } & =-0.65 \% & -0.743 & 7.84 \\
\text { STI DEV } & =0.002 & 0.046 & \\
\text { WATER FLOW } & =-1.331 & -1.852 & 41.37 \\
\text { STII DEV } & =0.002 & 0.205 & \\
\text { TOTAL FLOW } & =-2.020 & -2.6 .25 & 29.93
\end{array}
$$

PKL PROTDTYPE SFLIOL TEST RESLILTS

\section{TEST NO. UNITE (KO/S)} WYLE.

PYiL

\begin{tabular}{|c|c|c|c|c|c|}
\hline \multirow{3}{*}{$\begin{array}{l}0.0713 \\
0.5982\end{array}$} & DEG $C$ & NEWTIN & \multicolumn{2}{|c|}{ t.E/M*tra } & \multirow[b]{2}{*}{$F A(Y F A)=001.7$} \\
\hline & ----- & --ー-ー- & & --- & \\
\hline & $T F=145.0$ & {$[\mathrm{IF} 1=-4.771$} & $G[11=$ & 36.0 & $F[n(t: P A)=-1.564$ \\
\hline & $T W=141.7$ & $D F==-3,943$ & $\mathrm{Gi}[\mathrm{2}=$ & 7.7 & $F T(M / S)=-17.47$ \\
\hline
\end{tabular}

177

$\begin{array}{rlrl}\text { STEAM FLOW } & =-0.081 & -0.075 & -7.42 \\ \text { STD LEW } & =0.001 & 0.002 & \\ \text { WATER FLOW } & =-0.298 & -0.463 & 55.22 \\ \text { STD DEV } & =0.001 & 0.043 & \\ \text { TOTAL FLOW } & =-0.379 & -0.533 & 41.83\end{array}$

0.0000

LIEG $:$

NFWTIN

$k L / M+* 3$

ن. 1121

$\mathrm{TF}=144.7 \mathrm{UF} 1=-5.384 \quad 6 \mathrm{D} 1 \mathrm{l}=$

$T W=141.0 \mathrm{AF} \cdot 2=-5.1556 \mathrm{DL} 2=$

7.5 $F \Gamma(1: F A)=-1.509$

$T S=0.0[\mathrm{HF} 3=-0.193$ LOSS $=$

¿.4 FT (M/ $=-44.36$

$4.7 \quad \operatorname{l1T}(1,0, M-5)=-6002.9$
DEVIATION

AVERALE FKL TEST FAFIAHETERS
AUEFALE FKL TEST FAKANETERS

0.0063 DEG L NEWTON 1:G/THA3

0.1733

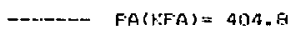

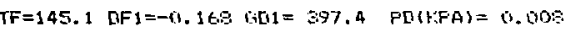
$T W=142.2$ nF $2=-0.093 \quad[1]=67.3 \quad \mathrm{FT}(\mathrm{M} / \mathrm{S})=-0.81$ TS= 0.0 [IF $S=0.001$ GDS: 3.3 LITT(KLIM-S)= $-4: 3.3$ 
FKL FRUTOTYFL SFOTL TEST RESLILTS

TEST NO. UNITS (KEGIS) WYLE PKL \% DIFF DEVIATIUN

179 STEAM FLOW $=-0.143-0.320$

STD DEV $=0.0010 .00 \mathrm{~s}$

WATER FLOW $=-8.400-7.471-11.05$

STD REV $=0.007 \quad 0.225$

TOTAL FLOW: $\quad-8.543 \quad-7.7 \% 1 \quad-2.00$
0.180 .4

DE.G C:

$0.9740 \quad T F=145.4 \quad[F(=-2.545$ DLI $=6.70 .9 \quad F[1(1: F \cdot A)=-1.072$ $T W=141.8$ DF $2=-2.435$ OLE $2=37 \% .6 \quad F T(M / S)=-2.83$

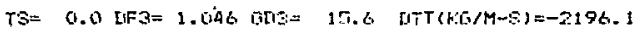

PKL PROTOTYFE SPDOLL TEST RESIILTS
TEST NO. LINITS (K'G/S) WYLE

F'KL

\% DIFF DEVIATION

RUERGLE FHI. TEST FARAMETEFS

179

$\begin{array}{rlrl}\text { STEAM FLOW } & =-0.124 & -0.089 & -23.30 \\ \text { STL DEV } & =0.003 & 0.003 & \\ \text { WATER FLOW } & =-0.823 & -0.469 & -43.07 \\ \text { STD IEV } & =0.003 & 0.026 & \\ \text { TOTAL FLOH } & =-0.947 & -0.557 & -41.14\end{array}$

0.0359

DEG $\mathrm{C}$

NEWTON

Ko $10+43$

-

0.3626

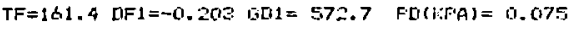

$T W=15 \mathrm{~F}, 1 \quad D F Z=-0.117$ GDZ $=201.7 \quad F T(M / S)=-0.70$

$T S=$

0.0 IFS 0.064 Gris:

4.1 DTT $(k: 0 / M-5)=-43.6$

FKL PROTOTYPE SFOOL TEST RESIILTS

$\begin{array}{rlrrr}\text { TEST NO. UNITS (KG/S) } & \text { WYLE } & \text { FKL } & \% \text { DIFF D } \\ 180 \text { STEAM FLOW } & =-1.392 & -1.090 & -0.13 \\ \text { STD NEV } & =0.057 & 0.007 & \\ \text { WATER FLIJW } & =-0.317 & -0.190 & -40.20 \\ \text { STD DEV } & =0.001 & 0.014 & \\ \text { TOTAL FLOW } & =-1.709 & -1.580 & -7.56\end{array}$

DEVIATIQN

0.0000

0.1309
AVERAGE FYLL TEST FARMMETEFS

TIEG E NEWTON TIGIMA:3

\section{-}

$T F=160,7$ [F $1=-5.435$ GD $1=$

3.7 $P D(K F A)=-1.573$

$T A=157,5$ DF $=-5,226$ BN2=

2.9 FT(M/F) $=-3 \mathrm{HS} .90$

$\mathrm{TS}=0.0 \mathrm{DF} 3=-0.169 \mathrm{ni} 13=$

$5.1 \quad \mathrm{UTT}(H: G / \mathrm{T}-S)=-6127.7$ 
PKL PROTOTYPE SPOOL TEST RESLILTS TEST NO. UNITS (KG/S) WYLE PK

1日2

$$
\begin{array}{rlrlr}
\text { STEAM FLOW } & =-0.510 & -0.489 & -4.09 \\
\text { STI DEV } & =0.006 & 0.029 & \\
\text { WATE, FLOW } & =-0.595 & -1.303 & 118.92 \\
\text { STD DEV } & =0.002 & 0.151 & \\
\text { TOTAL FLLW } & =-1.1015 & -1.792 & 62.15
\end{array}
$$

DEVIATION

0.0344

0.7377

.737
FKL PROTOTYPE SPOOL TEST FESULTS

\begin{abstract}
TEST INO. UNITS (KG/S)
\end{abstract}
HYLE

Pl:

192

$\begin{array}{rlrl}\text { STEAM FLOW } & =-0.312 & -0.504 & 61.63 \\ \text { STD DEV } & =0.003 & 0.009 & \\ \text { WATER FLOW } & =-5.537 & -5.477 & -0.73 \\ \text { STD DEV } & =0.006 & 0.210 & \\ \text { TOTAL FLOW } & =-5.849 & -6.001 & 2.60\end{array}$

DEVIATION

0.1764

0.2139 $T W=155.1$ LF $2=-2.510 \quad G[2=157.0 \quad F T(M / 3)=-4.14$ $T S=0.0$ DF3 = 0.36S GDS= $\quad 0.7$ [ITT $(K[. / H-3)=-2474.2$
AVERALE FH:L TEST FARAMETEFS

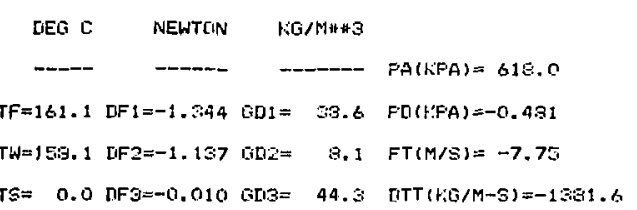

PKL 'PROTOTYFE SPOOL TEST RESULTS TEST NO. UNITS (KG/S) WYLE PKL * \# RIFF DEVIATION

-

$189 \quad \begin{array}{rlrl}\text { STEAM FLOW } & =-0.043 & -0.034 & -21.91 \\ \text { STD DEV } & =0.001 & 0.003 & \\ \text { WATER FLOW } & =-0.590 & -0.5 / 7 & -3.61 \\ \text { STO DEV } & =0.001 & 0.068 & \\ \text { TOTAL FLOW } & =-0.642 & -0.611 & -4.83\end{array}$

0.0101 
FKL PROTOTYPE SFOOL TEST RESIILTS

TEST NO. UNITS $(K 0 / S)$ WYLE

Fk'L * JIFF DEVIATION

189

\subsection{TEG L NEWTON RIG/MU\#}

-

0.8740 TF=121.1 [FF $1=-0.0 .69$ GH $1 \approx 112.9 \quad F\left[\left(1 . P A_{1}\right)=-0.202\right.$ $T W=113.4$ DF $2=-0.545[5 / 2=28.0$ FT $(M / S)=-3.92$ $T S=0.0$ IJFS $=0.531 \quad[L E S=$

4.7 ITT $(K, G / M-s)=-552.4$

PKL PRATOTYFE SPOQL TEST RESIILTS

TEST NO. UNITS (F,L/S) WYLE

TEST

190
DEVIATION

0.0959

0.7181

WATER FLOW $=-5.752-5.083-11.63$

STD DEV =

0.0155

D. 222

TOTAL. FLOW $=$

$-5.95$

$-5.28$

$-9.82$
FKL PROTOTYPE SPOOI. TEST RESULTS TEST NO. UNITS (KG/S) WYLE PKIL \% DIFF DEVIATIDN 191

$\begin{array}{rlrl}\text { STEAM FLOW } & =-0.710 & -0.822 & 15.61 \\ \text { STD DEV } & =0.001 & 0.009 & \\ \text { WATER FLOH } & =-0.602 & -0.489 & -18.83 \\ \text { STD DEV } & =0.001 & 0.031 & \\ \text { TOTAL FLOW } & =-1.312 & -1.311 & -0.09\end{array}$

\section{AUERALIE FKR TEST FARAMETERS}

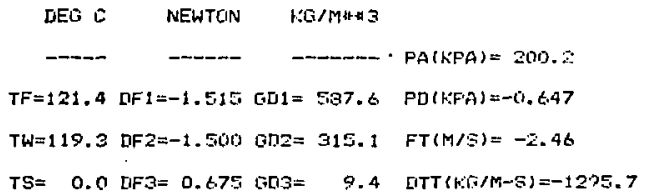


FKL PROTOTYFE SFOOL TEST RESULTS

$\begin{array}{rlrlrl}\text { TEST NO. UNITS (KO/S) } & \text { WYLE } & \text { PKL } & \% \text { DIFF } & \text { IIEVIATIUN } \\ 192 & \text { STEAM FLOW } & =-0.136 & -0.302 & 121.91 & 0.1633 \\ \text { STD IIEV } & =0.002 & 0.005 & & \\ \text { WATER FLLW } & =-0.236 & -7.134 & -13.90 & 1.2082 \\ \text { STD IEV } & =0.007 & 0.278 & & \end{array}$

AVERAGE FKI. TEST FARIFNETERS

\begin{tabular}{|c|c|c|c|}
\hline GEG $C$ & NEWTON & $\mathrm{H}: \mathrm{L} / \mathrm{M} * * 3$ & \\
\hline$-\infty$ & - & - & $F A(1 ; P A)=414.2$ \\
\hline$F=144.3$ & {$[A F 1=-2.217$} & $651=696.9$ & $F Q(1 . P A)=-0.071$ \\
\hline$W=142.6$ & $\mathrm{OF} 2=-2.198$ & $15 \square 2=3 \% 5.0$ & $F T(H / S)=-2.62$ \\
\hline
\end{tabular}

PKL FROTOTYPE SPOOL TEST RESLILTS

TEST NO. UNITS (KG/S) WYLE PH:L

\% DIFF DEVIATION

193

AVEFAGE FKLL TEST FAFAMETER:
0. 0000

LIEO :

10.0. -

0.1397 $T W=142.8$ iF $2=-5.309$ on 20 $T S=0.0$ DF $3=-0.195 .0 D 3=$

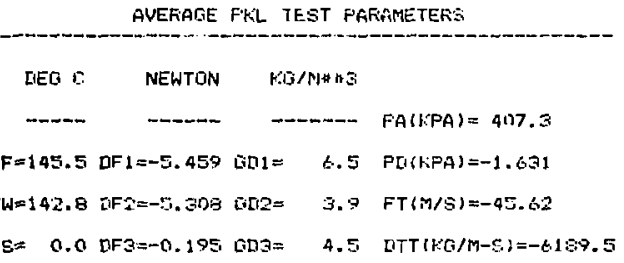

AVERAGE FNL TEST FARAMETERE

PKL PROTOTYFE SFGOL TEST RESLILTS

TEST NO. UNITS QKO/S) WYLE PKL \% DIFF LIEUIATION

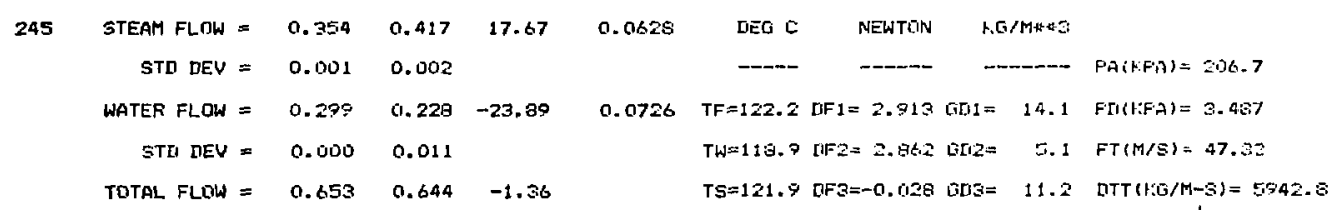


PKL PROTOTYFE EPCINL TEST RESILILTS

\begin{tabular}{|c|c|c|c|c|c|c|c|c|c|c|c|c|c|}
\hline ST NO & LINITS (KLISI) & & WYLE & FKL & \% חIfF & REVIATION & \multicolumn{7}{|c|}{ AVERAGE FRIL TEST FARAMETERS } \\
\hline 249 & STEAM FLOW & $=$ & 0.054 & 0,518 & 46.21 & 0.1670 & DEG $\mathrm{C}$ & & NEWTON & & $3 / m \times 3$ & & \\
\hline & STH DEV & $=$ & 0.003 & 0.005 & & & $--\infty$ & & $\rightarrow-\infty$ & - & $-\infty-\infty$ & $P A(K P A)=$ & 421.2 \\
\hline & WATER FLEW & $=$ & $0 . \sin 2$ & 0.588 & -14.18 & 0.2076 & $T F=146.3$ & $D F I=$ & 2.530 & $\mathrm{GnI}=$ & 25.1 & $F D(K: F A)=$ & 3.318 \\
\hline & STR DEV & $=$ & 0.004 & 0.049 & & & $T W=143.4$ & $D F:=$ & $=2.549$ & $602=$ & 12.3 & $\operatorname{FT}(M / S)=$ & 24.92 \\
\hline & TOTAL FLOW & $=$ & 1.016 & 1.096 & $b .87$ & & $T S=148.1$ & $\mathrm{IIFS}=$ & $=0.0119$ & $6013=$ & 22.4 & עTT H:LiM. & $\left.-c_{i}\right)=5.279 .4$ \\
\hline
\end{tabular}

PKL PROTOTYPE SPUOL TEST FESULTS

TEST NO. UNITS (KG/S) WYLE

WYLE

FKL \% IIFF DEVIATION

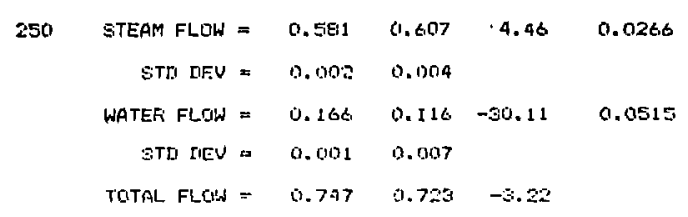

PKL PROTOTYPE SFGOL TEST RESLILTS

TEST NO. UNITS (KOTS)

WYLE

\% DIFF DEVIATION

0.025

PKL \% DIFF DEVIATION

251

\begin{tabular}{|c|c|c|c|c|c|}
\hline \multirow[t]{5}{*}{251} & STEAM CLOW = & 0.025 & 0.057 & 127.36 & 0.0325 \\
\hline & STI DEV = & 0.001 & 0.002 & & \\
\hline & WATEF FLOW $=$ & 7.350 & 5.693 & -19.92 & 1. Egege \\
\hline & STD DEV = & 0.0105 & 0.195 & & \\
\hline & TOTAL FLOW = & 7.354 & 5.450 & -19.42 & \\
\hline
\end{tabular}

AVERAGE FKL TEST FARAMETERS.

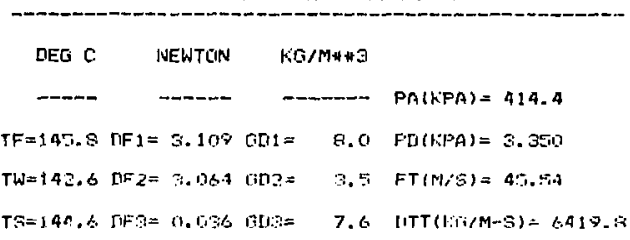

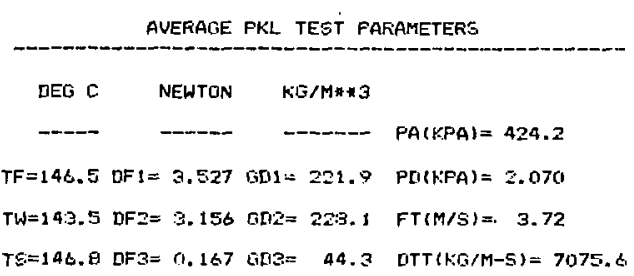


FKL PROTOTYPE SFDOL TEST FESLILTS TEST NO. UNITS (KO/S) WYLE PKL \% DIFF DEVIATION

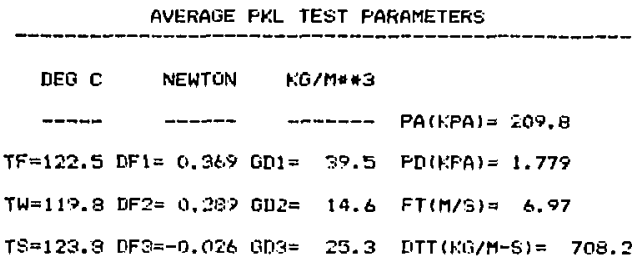

PKL FROTOTYFE SPIOIL TEST RESIILTS

\begin{abstract}
TEST NO. UNITS ( $K$ CO/S)
\end{abstract} WYLE

FKL

$\%$ DIFF DEVIATION

259
$0.118 \quad 59.69$

0.0451

b. 102

WATER FLOH =

STD NEV =

TOTAL FLOW =
0.403

35.75

0.039

$0.521 \quad 40.52$
0.1147

$T S=123.9$ DFO=-0.026 GnI

AVERATSE PKL TEST F'ARAMETERS

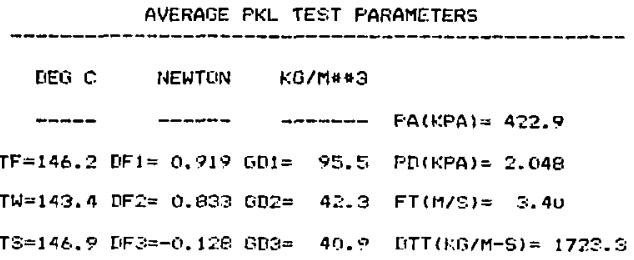

FKL PFITTOTYPE SPODL TEST RESULTS

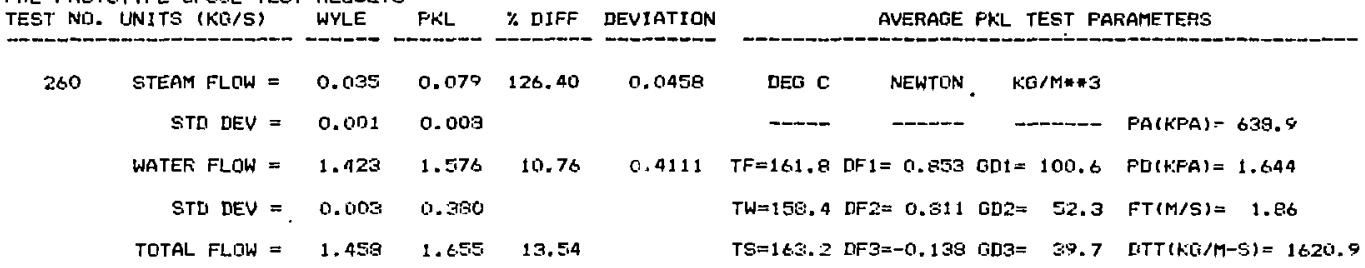


PKL PRQTOTYFE EPQOL TEST RESULTS

TEST NO. UNITS (KG/S) WYLE

PKL. $\%$ IIFF

DEVIATION

$$
261
$$

$$
\begin{array}{r}
\text { STEAM FLOW }= \\
\text { STD DEV }=
\end{array}
$$

0.104

$0.094 \quad-9.28$

0.0157

0.002

0.012

WATER FLEIW $=1.4$ ĖB

$1.879 \quad 26.26$

STII LIEV $=0.003$

0. 421

TOTAL FLOW $=1.502$

1.973

23.93
AVERAGE PN:L TEST FARAMETEFS

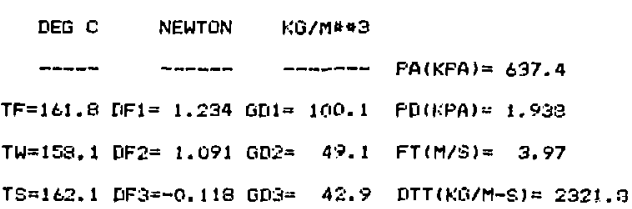

\section{AUERAGE PR:L TEST FARAMETEKS}

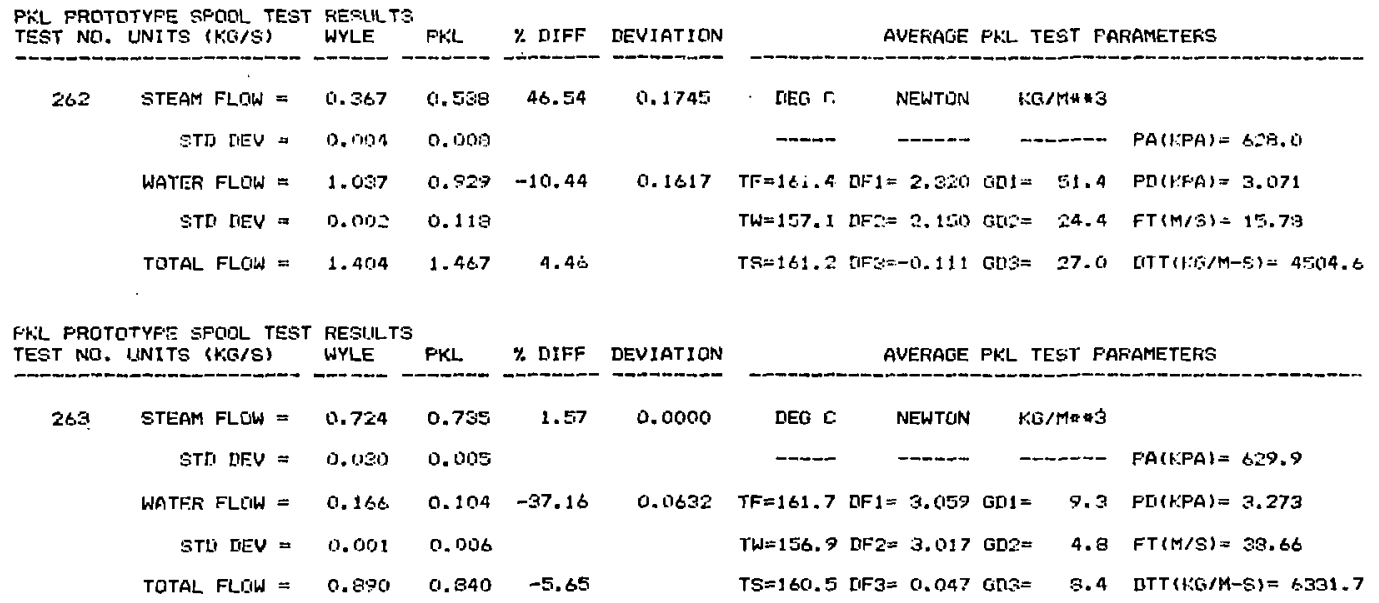


FYL PRLTITYPE SPODL TEST RESULLTS

TEST NO. UNITS (KIG/S) WYLE

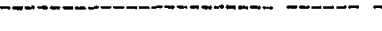

262

$\begin{array}{rlrl}\text { STEAM FLOIW } & =-0.359 & -0.497 & 21.83 \\ \text { STR DEV } & =0.001 & 0.011 & \\ \text { WATER FLOW } & =-0.301 & -0.333 & 10.52 \\ \text { STD DEV } & =0.002 & 0.052 & \\ \text { TOTAL FLOW } & =-0.660 & -0.770 & 16.67\end{array}$

0. 0807

(2)

0.0615

$T F=121.9 \quad$ IF $1=-3.347$ Git $1=$

$T W=-0.0$ UF $2=-3.339$ CID2=

$T S=123.2: n F 3=0.291 \quad[163=$

AVERAGE PKL TEST PARAMETERS

\begin{tabular}{|c|c|c|c|c|}
\hline DEG C & NEWTAN & \multicolumn{2}{|c|}{$N G / M * 3$} & \\
\hline$-\infty-\infty$ & ---- & \multicolumn{2}{|c|}{--m-n- } & $F A(K, P A)=203 . \theta$ \\
\hline$T F=121.9$ & $\mathrm{DF} 1=-3.347$ & GIt $1=$ & 6.2 & $P D(1, P A)=-0.003$ \\
\hline$T W=-0.0$ & $\angle I F 2=-3.339$ & $0,02=$ & 5.7 & $F T(M / S)=-47.48$ \\
\hline$T S=123.2$ & $n F 3=0.291$ & $5013=$ & 6.5 & DTT $(1: G / M-S)=-7101.0$ \\
\hline
\end{tabular}

PFL FROTUTYFE BFCOL TEST RESULTS
TEST NO. UNITS (KG/S) WYLE FNL \% RIFF DEVIATION

-

283

$$
\begin{array}{rlrl}
\text { STEAM FLOW } & =-0.180 & -0.217 & 20.36 \\
\text { STD DEV } & =0.004 & 0.022 & \\
\text { WATER FLOW } & =-0.697 & -1.044 & 5.03 \\
\text { STO NEV } & =0.002 & 0.140 & \\
\text { TOTAL FLUW } & =-0.967 & -1.261 & 45.46
\end{array}
$$

\section{AVERAGE FKL TEST PARAMETERS}

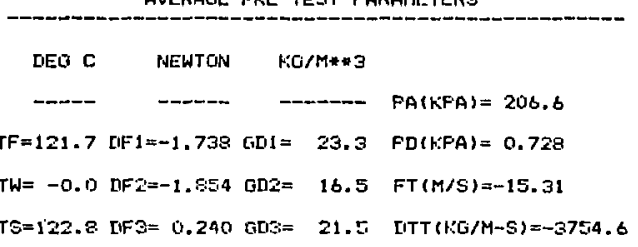

FKL FROTOTYPE SFONL TEST REGULTS

TEST NO. LINITS (KO/S) WYLE

FH:L

\% DIFF DEVIATION

AUEFAGE FHL TEST PARAMETERS

234

$$
\begin{array}{rlrrr}
\text { STEAM FLIW } & =-0.071 & -0.105 & 47.36 \\
\text { STU HEV } & =0.001 & 0.005 & \\
\text { WATEA FLUW } & =-0.303 & -0.253 & -16.61 \\
\text { STI LEV } & =0.001 & 0.073 & \\
\text { TOTAL. FLUW } & =-0.374 & -0.557 & -4.46
\end{array}
$$

0.0347

IIEG C NEWTON H:G/MHA

$\cdots$

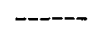

-

$0.0895 T F=122.2 \mathrm{nF} I=-0.174$ GnI $=114.7 \quad \mathrm{P}[(\mathrm{FPA})=1.312$

$T W=0.0$ nF $2=-0.287$ GD2 $=63.7 \quad F T(M / 5)=-6.16$

$T S=133.2$ LIF $3=0.016$ GDS= 44.3 DTT $(1.5 / M-S)=-420.3$ 
PKL PRDTOTYPE SPODL TEST RESULTS

TEST NO. UNITS (KG/S) WYLE PKL \% DIFF LIEVIATION

285 STEAM FLON $=-0.071 \quad-0.055 \quad-22.87 \quad 0.01391$

STD DEV $=0.001 \quad 0.035$

WATER FLOW $=-1.554 \quad-1.827$

17.60

0.5294

STD DEV $=0.009 \quad 0.449$

TOTAL FLOW $=-1.625-1.862 \quad 15.83$
AVERAGE PKL TEST PARAMETERS

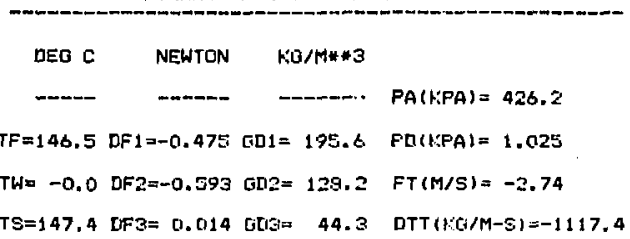

PKL PROTOTYPE SPOILL TEST RESLILTS

TEST NO. UNITS (KGIS) WYLE

WYLE F'KL

XIFF

DEVIATION

286

$$
\text { STEAM }
$$

$$
\text { STD DEV }=0.001
$$

$-0.033$

37.73

0.0094

0.002

WATER FLUH $=-7.34$

$-6.777 \quad-7.49$

D. 300

TOTAI_ FLOW $=-7.350-8.6110$
STD DEV $=0.00$

AVERAGE PKL TEST FARAMETERS

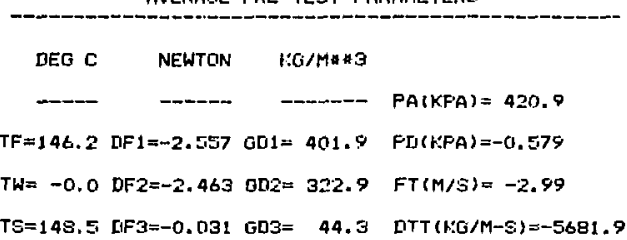

PKL PROTOTYFE SFOOL TEST RESILTS TEST NO. UNITS (KO/S) 287 WYLE PK'L \% DIFF DEVIATION

\section{AVERAGE FK:L TEST F'ARAMETERS}

AVERAGE FK:L TEST FARAMETERS
-

\subsection{DEG C NEWTON $\mathrm{KG/M \# 3}$}

$\begin{array}{rlrlr}\text { STD DEV } & =0.002 & 0.060 & \\ \text { WATER FLOW } & =-0.604 & -0.361 & 25.84 \\ \text { STD DEV } & =0.001 & 0.159 & \\ \text { TOTAL FLIDW } & =-1.039 & -1.310 & 26.09\end{array}$

$0.2402 T F=146.9$ [F $1=-2.500 \quad \mathrm{GD} 1=15.6$ FD(1:PA) $=0.277$ TW $=-0.0$ DF2 $=-2.621$ LILZ= 14.5 FT $(M / S)=-21.56$

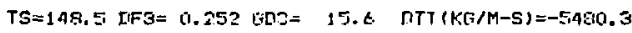


PKL FROTOTYPE SFOOL TEST FEGIILTS TEST NO. UNITS (KOL/S) WYI.E FKL \% RIFF LEVIATION

2as

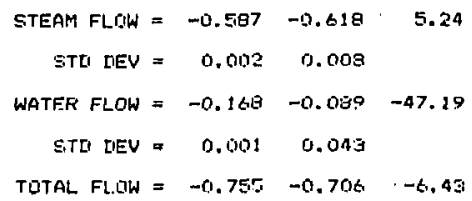

FHL PROTOTYPE SPENDL TEST RESUILTS TEST NOE. UNITS (H:G/S)

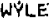

PKL FROTETTYPE SPEIOL TEST RESIJLTS

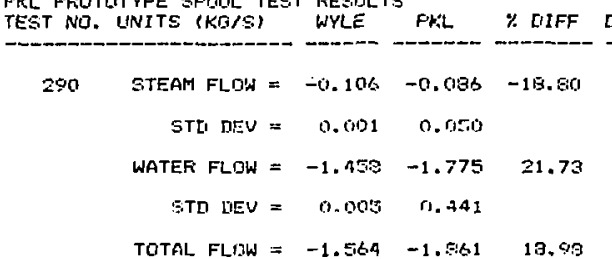

AVERALEF FH'L TEST PAFAMETEFS

\section{NEWTON NOSIME+}

$P A(F P A)=644.0$

(2)
DEVIATION

0.0540

0.5470

Tas

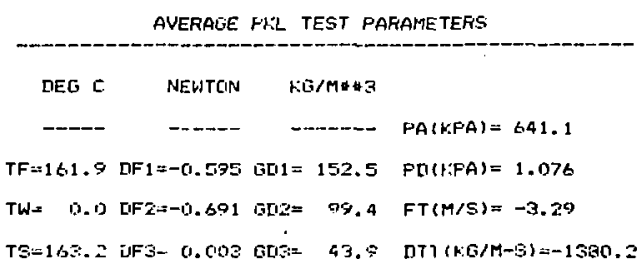


FILL FRITITYFE SFIOL. TEST RESILTS TEST NO. UNITS (KG/E) WYLE

EKL \% DIFF

DEVIATION

291

0.1339
0.3902

0.3942

\section{$T$}

TOTOL FLOW $=-1.413-1.857 \quad 34.26$

PKL PROTOTYFE SPIOL TEST RESLILTS

TEST ND, UNITS (KE/S) WVLE FFIL \% IIJFF DEUIATION

(1) - -

$\begin{array}{rlrlrl}292 & \text { STEAM FLOW } & =-0.710 & -0.729 & 2.65 & 0.0712 \\ \text { STL DEV } & =0.002 & 0.069 & & \\ \text { WATER FLDW } & =-0.157 & -0.073 & -53.35 & 0.1735 \\ \text { STO LIEV } & =0.001 & 0.151 & & \\ \text { TCITAL FLOW } & =-0.867 & -0.802 & -7.49 & \end{array}$

FKL FROTOTYFE SPOOL TEST RESIILTS

$$
\text { T }
$$

240
WYLE FKL \% IIFF DEVIATIDN

$-0.076 \quad-0.116 \quad 52.53$

0.0010 .005

ZTD DEV $=0.001$

0.006

WATER FLOW $=-0.30 \%-0.27$

STI MEV $=0.0020 .071$

TOTAL FLOW $=-0.395-0.375$
$-9.71 \quad 0.0771$

2.53

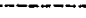

0.0412
$-1) .0$ DF2 $=-2.54 \% \mathrm{GL} 2=24.4 \quad F T(M / S)=-16.93$

AVERAGE PKL TEET PARAMETERS

\begin{tabular}{|c|c|c|c|c|}
\hline DEG C & NEWTON & & IM\#可 & \\
\hline - & $-\infty$ & & $-\infty$ & $P A($ K.F'A $)=638.6$ \\
\hline$=161.7$ & $\mathrm{DF}_{1}=-2.950$ & {$[\because D 1=$} & 29.0 & $F D(1, F \cdot A)=0.0 .6$ \\
\hline$=-1.0$ & $D F 2=-2,848$ & $002=$ & 24.4 & $F T(M / S)=-16.93$ \\
\hline
\end{tabular}

\begin{tabular}{|c|c|c|c|c|}
\hline DEG $c$ & NEHTON & & $\mathrm{K} Q \mathrm{C} / \mathrm{M} *=3$ & \\
\hline - & $-\infty-\infty$ & & $--\infty-\infty$ & $P A(1: P A)=643.3$ \\
\hline$T F=162.1$ & {$[|F|=-2.907$} & $\mathrm{GO}_{1}=$ & 5.5 & $P T_{1}(1: P A)=0.336$ \\
\hline$T W=-1) .3$ & $D F 2=-2.854$ & $\mathrm{an} 2=$ & 6.6 & $F T(M / S)=-42.50$ \\
\hline$T S=16 \dot{\hat{L}} . \theta$ & {$[1 F 3=0.0155$} & & 6.5 & DITT $(1: B / M-S)=-6$ \\
\hline
\end{tabular}
AVERALIE PI:L. TES'T FARAMETEKS:

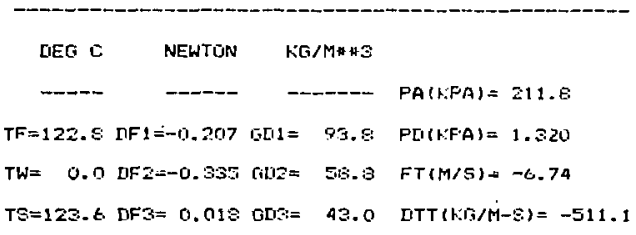


PKL PFIOTOTYPE SFOOL TEST RESIILTS

TEOT NO. UNITS (h:0/S)

WYLE FHL \% DIFF DEVIATION

299 STEAM FLOW $=-0.072 \quad-0.038 \quad-47.28 \quad 0.0348$

STD IIEV $=0.01 .4 \quad 0.0104$

WATEF FLOW $=-1.449-2.022 \quad 39.61$

STD DEV $=0.0 \%$. 0.244

TOTML. FLOW $=-1.520-2.040 \quad 25.50$
AVERAGE PHIL. TEST PARAMETERS

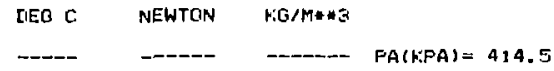

$T S=146.7$ [.F $2=0.014$ G. 